\title{
PARAMETERS GOVERNING SEPARATION CONTROL WITH SWEEPING JET ACTUATORS
}

by

\author{
René Woszidlo
}

\author{
A Dissertation Submitted to the Faculty of the \\ Department Of Aerospace And Mechanical EngineERING \\ In Partial Fulfillment of the Requirements \\ For the Degree of \\ DOCTOR OF PHILOSOPHY \\ WITH A MAJOR IN AEROSPACE ENGINEERING \\ In the Graduate College \\ THE UNIVERSITY OF ARIZONA
}




\section{THE UNIVERSITY OF ARIZONA GRADUATE COLLEGE}

As members of the Dissertation Committee, we certify that we have read the dissertation prepared by Rene Woszidlo entitled "Parameters Governing Separation Control with Sweeping Jet Actuators" and recommend that it be accepted as fulfilling the dissertation requirement for the Degree of Doctor of Philosophy

Dr. Israel Wygnanski

Date: $10-28-2011$

Date: $10-28-2011$

Dr. Anatoli Tumin

Date: $10-28-2011$

Dr. Jesse Little

Final approval and acceptance of this dissertation is contingent upon the candidate's submission of the final copies of the dissertation to the Graduate College.

I hereby certify that I have read this dissertation prepared under my direction and recommend that it be accepted as fulfilling the dissertation requirement.

Dissertation Director: Dr. Israel Wygnanski

Date: $10-28-2011$ 


\section{STATEMENT BY AUTHOR}

This dissertation has been submitted in partial fulfillment of requirements for an advanced degree at The University of Arizona and is deposited in the University Library to be made available to borrowers under rules of the Library.

Brief quotations from this thesis are allowable without special permission, provided that accurate acknowledgment of source is made. Requests for permission for extended quotation from or reproduction of this manuscript in whole or in part may be granted by the head of the major department or the Dean of the Graduate College when in his or her judgment the proposed use of the material is in the interests of scholarship. In all other instances, however, permission must be obtained from the author.

SIGNED: René Woszidlo 


\section{ACKNOWLEDGEMENTS}

I would like to acknowledge and thank the following people for their contributions and support. Without them this work would have not been possible.

First and foremost I would like to thank my advisor Dr. Israel Wygnanski. His scientific expertise and sharp intellect provided tremendous input and guidance to my work. His frequent "push" was often helpful in keeping me going. I am also grateful for his friendly advice and wisdom.

Besides the scientific part of my work, the design and build of my experiments would have been impossible without the machine shop icon Dale Drew. Not only am I thankful for his work but also for personal conversations which often provided a needed distraction. Thank you, Dale, for being a helpful colleague and an honest friend.

I want to thank the members of the Aerolab (i.e., Roman Seele, Philipp Tewes, Elisa Philips, Nick Zalewski, Stephen Beck, Nathaniel Lucas, Holger Nawroth, and Lutz Taubert) for being supportive colleagues and friends. Their contributions to my work and the regular lunch breaks were invaluable to overcome many stressful times.

Many friends were helpful in balancing the time spent at school. In particular, I would like to thank my host family, Gail and Jan Jager, for their support and delicious meals. They were a needed family away from home.

Last, I want to thank the entire staff from the Department of Aerospace and Mechanical Engineering at the University of Arizona for providing a supportive and professional structure for the education and work of all the students. 


\section{SPONSOR ACKNOWLEDGEMENTS}

I would like to acknowledge the financial support received under a NASA Phase II SBIR grant to Advanced Fluidics LLC. I would also like to thank Dr. Surya Raghu from Advanced Fluidics LLC for his scientific contributions and advice.

\section{DEDICATION}

To my Mom and Dad whose endless love and support made me become the person I am today and have allowed me to pursue my dreams while knowing that somebody is standing behind me.

To my lovely wife, Alesia, who has guided my way to the $\mathrm{PhD}$ by example and supported me with her love and understanding on this challenging journey. I love you! 


\section{TABLE OF CONTENTS}

LIST OF FIGURES

LIST OF TABLES.

ABSTRACT

NOMENCLATURE 14

1 INTRODUCTION. 17

1.1 Research Objectives .21

2 EXPERIMENTAL SET-UP AND INSTRUMENTATION 23

3 PARAMETER OVERVIEW AND DISCUSSION 30

4 HOW SHOULD ACTUATION BE APPLIED? ................ 38

4.1 Characterization of Actuators ............................................38

4.2 Effects of Reynolds Number ...............................................48

4.3 Effects of Hysteresis.......................................................49

4.4 Quantification of Actuation Intensity .................................52

4.5 Surface Flow Visualization ..................................................58

4.6 Sweeping vs. Non-Sweeping Jets ....................................64

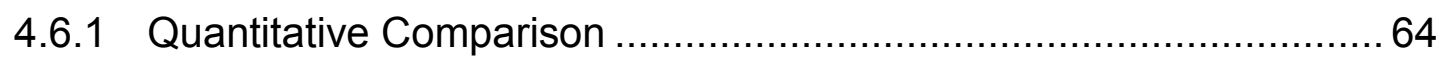

4.6.2 Qualitative Comparison ........................................................... 71

5 WHERE SHOULD ACTUATION BE APPLIED? ........... 78

5.1 Actuation from a Single Location ........................................... 


\section{TABLE OF CONTENTS - Continued}

5.2 Actuation from Multiple Locations ....................................87

\section{ON WHAT AIRFOIL CONFIGURATION SHOULD}

ACTUATION BE APPLIED? ........................................ 90

6.1 Comparisons between Numerical and Experimental Data .90

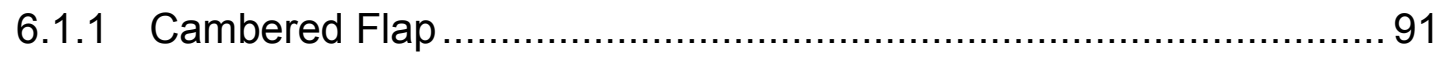

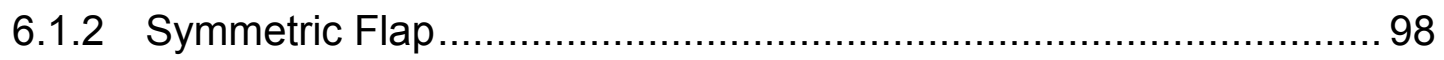

6.2 Effects of Flap Deflection ................................................. 104

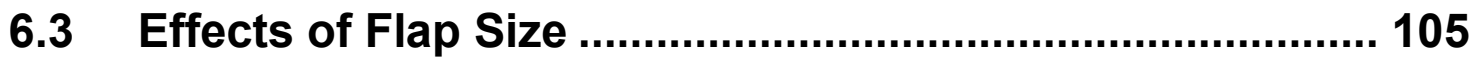

6.4 Effects of Angle of Attack ........................................... 112

7 HOW CAN ACTUATION BE SCALED? ................... 118

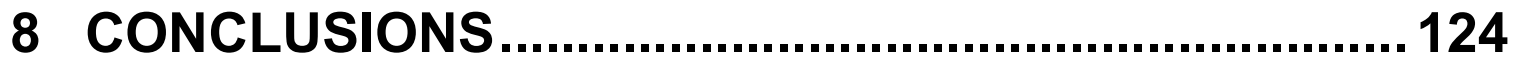

9 FUTURE WORK ................................................... 130

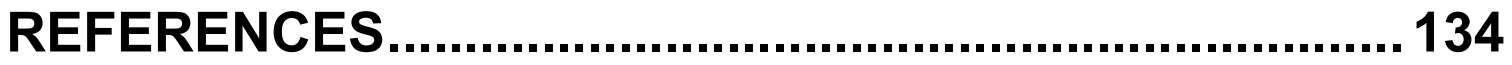




\section{LIST OF FIGURES}

Figure 1 - Conceptual actuator designs: a) discrete jet outlets ${ }^{[40]}$, b) continuous sweeping range $^{[39]}$.

Figure 2 - Design of Multiple Flap Airfoil (MFA). ....................................................... 23

Figure 3 - Various flap sizes and shapes tested with MFA model................................. 24

Figure 4 - Complete assembly of MFA in 20\% flap configuration. .............................. 27

Figure 5 - Time series (top) \& spectrum (bottom) of exemplary sweeping jet actuator,

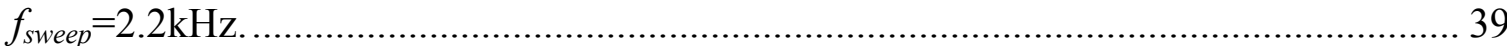

Figure 6 - Sweeping frequency vs. volume flowrate per actuator for various $\Delta z$.......... 40

Figure 7 - Ratio of measured \& calculated jet momentum vs. flowrate per actuator. .... 43

Figure 8 - Approximated hotwire and load cell time signals. ...................................... 44

Figure 9 - Calculated force ratio as a function of sweeping angle............................. 47

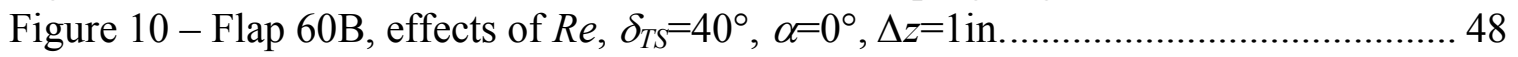

Figure 11 - Flap 40A, hysteresis of changing $C_{\mu}, \delta_{T S}=45^{\circ}, \alpha=0^{\circ}$, AFC @ 7\% $c_{\text {flap }}$,

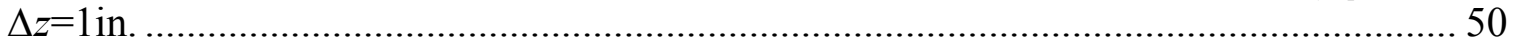

Figure 12 - Flap 40A, hysteresis of changing $\alpha, \delta_{T S}=40^{\circ}$, AFC @ 7\% $c_{\text {flap }}, \Delta z=1$ in...... 52

Figure 13 - Flap 40B, required $C_{\mu} \& C_{Q}$ for fixed $C_{l}=1.5, \alpha=0^{\circ}$, AFC @ 4\% $c_{\text {flap }} . . . . . . .53$

Figure 14 - Flap 40B, actuation intensity for various $\Delta z, \alpha=0^{\circ}$, AFC @ 4\% c flap .......... 54

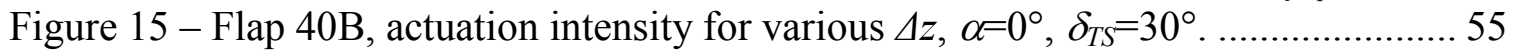

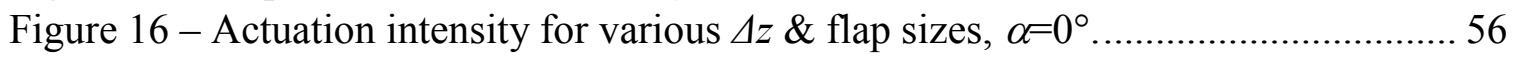

Figure 17 - Surface flow visualization, $\alpha=0^{\circ}, \delta_{T S}=30^{\circ}$, baseline................................. 59

Figure 18 - Surface flow visualization, $\alpha=0^{\circ}, \delta_{T S}=30^{\circ}$, AFC @ $0 \% c_{\text {flap }}, C_{\mu}=3 \% \Delta z=1$ in.,

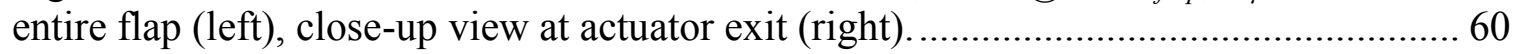

Figure 19 - Surface flow visualization, $\alpha=0^{\circ}, \delta_{T S}=30^{\circ}$, AFC @ $0 \% c_{\text {flap }}, C_{\mu}=3 \%, \Delta z=1$ in., streamwise streaks down to flap TE. ........................................................................ 61

Figure 20 - Surface flow visualization, $\alpha=0^{\circ}, \delta_{T S}=30^{\circ}$, AFC @ $0 \% c_{\text {flap }}, C_{\mu}=6 \%, \Delta z=1$ in., entire flap (left), close-up view at actuator exit (right)............................................... 63

Figure 21 - Flap 20A, lift vs. actuation intensity, $\alpha=0^{\circ}$, non-sweeping jets, AFC @ 18\%

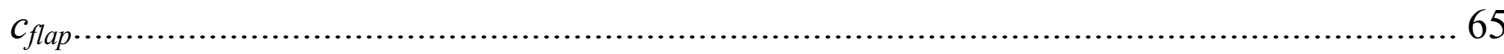

Figure 22 - Flap 20A, lift vs. flowrate coefficient, $\alpha=0^{\circ}$, non-sweeping jets, AFC @ 18\%

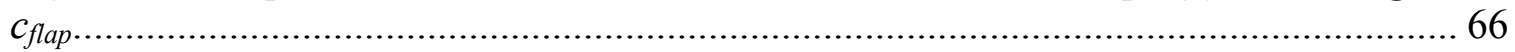

Figure 23 - Flap 20A, hysteresis of changing $C_{\mu}, \delta_{T S}=40^{\circ}, \alpha=0^{\circ}$, AFC @ 20\% $c_{\text {flap }}$, $\Delta z=1$ in.,

Figure 24 - Flap 20A, sweeping vs. non-sweeping jets with $\left(C_{d t}+C_{\mu}\right)_{\min }$, AFC @ 20\%

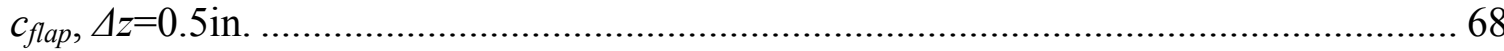

Figure 25 - Flap 20A, sweeping vs. non-sweeping jets with $\left(C_{d t}+C_{E}\right)_{\min }$, AFC @ 20\%

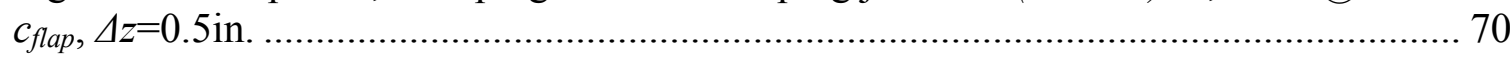

Figure 26 - Flap 20A, surface flow visualization, no free-stream, AFC @ 20\% $c_{\text {flap }}$, entire flap, left: non-sweeping jets, right: sweeping jets, A) $\Delta z=0.5$ in., $Q \leftrightarrow C_{\mu}=3 \% @$ $\left.R e=6 \cdot 10^{5}, \mathrm{~B}\right) \Delta z=1$ in., $\left.Q \leftrightarrow C_{\mu}=6 \% @ R e=6 \cdot 10^{5}, \mathrm{C}\right) \Delta z=1.5$ in., $Q \leftrightarrow C_{\mu}=4.5 \% @ R e=6 \cdot 10^{5}$. 


\section{LIST OF FIGURES - Continued}

Figure 27 - Flap 20A, surface flow visualization, $\alpha=0^{\circ}, \delta_{T S}=40^{\circ}$, AFC @ 20\% $c_{\text {flap }}$, entire flap, left: non-sweeping jets, right: sweeping jets, A) $\Delta z=0.5 \mathrm{in} ., C_{\mu}=3 \%$, B) $\Delta z=1$ in., $C_{\mu}=6 \%$, C) $\Delta z=1.5$ in., $C_{\mu}=4.5 \%$.

Figure 28 - Flap 20A, surface flow visualization, $\alpha=0^{\circ}, \delta_{T S}=40^{\circ}$, AFC with nonsweeping jets @ 20\% $c_{\text {flap }}, \Delta z=1$ in., $C_{\mu}=6 \%$, close-up view of actuator outlet. 75 Figure 29 - Flap 20A, surface flow visualization, $\alpha=0^{\circ}, \delta_{T S}=40^{\circ}$, AFC with sweeping jets (a) $\sim 20 \% c_{\text {flap }}, \Delta z=1.5 \mathrm{in}$., $C_{\mu}=4.5 \%$, entire flap. 77

Figure 30 - Flap 40B, lift vs. actuation location, $\alpha=0^{\circ}, \Delta z=1$ in................................... 78

Figure 31 - Flap 40B, lift vs. actuation location, $\alpha=0^{\circ}, \delta_{T S}=40^{\circ}$............................... 80

Figure 32 - All flaps, lift vs. actuation location, $\alpha=0^{\circ}, \delta_{T S}=40^{\circ}, \Delta z=1$ in. ..................... 81

Figure 33 - Flap 40A, lift \& total drag vs. $C_{\mu}, \alpha=0^{\circ}, \delta_{T S}=0^{\circ}, \Delta z=1$ in. .......................... 83

Figure 34 - Flap 40A, $C_{p}$-distributions \& wake profiles, $\delta_{T S}=0^{\circ}, \alpha=0^{\circ}, C_{\mu}=6 \%, \Delta z=1$ in. 85

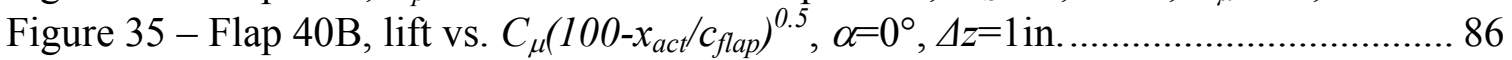

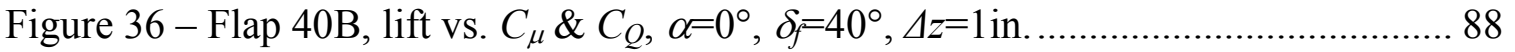

Figure 37 - Flap 40B, lift and moment vs. $\delta_{T S}, \alpha=0^{\circ}$, AFC @ 4\% $c_{\text {flap }}, \Delta z=1$ in............. 91

Figure 38 - Flap 40B, drag vs. $\delta_{T S}, \alpha=0^{\circ}$, AFC @ 4\% $c_{\text {flap }}, \Delta z=1$ in. ............................. 92

Figure 39 - Flap 40B, $C_{p}$-distributions, $\delta_{T S}=20^{\circ}, \alpha=0^{\circ}$, AFC @ 4\% $c_{\text {flap }}, \Delta z=1$ in.......... 94 Figure 40 - Flap 40B, $C_{p}$-distributions $\&$ wake profiles, $\delta_{T S}=30^{\circ}, \alpha=0^{\circ}, \mathrm{AFC} @ 4 \% c_{\text {flap }}$,

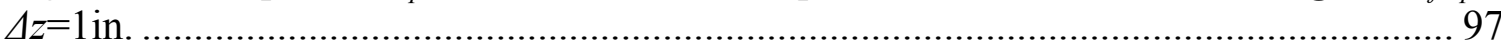

Figure 41 - Flap 40B, $C_{p}$-distributions, $\delta_{T S}=40^{\circ}, \alpha=0^{\circ}$, AFC @ 4\% $c_{\text {flap }}, \Delta z=1$ in.......... 98

Figure 42 - Flap 40A, lift and moment vs. $\delta_{T S}, \alpha=0^{\circ}$, AFC @ 7\% $c_{\text {flap }}, \Delta z=1$ in............. 99

Figure 43 - Flap 40A, drag vs. $\delta_{T S}, \alpha=0^{\circ}$, AFC @ 7\% $c_{\text {flap }}, \Delta z=1$ in.............................. 100

Figure 44 - Flap 40A, $C_{p}$-distributions, $\delta_{T S}=0^{\circ}, \alpha=0^{\circ}$, AFC @ 7\% $c_{\text {flap }}, \Delta z=1$ in........... 101

Figure 45 - Flap 40A, $C_{p}$-distributions, $\delta_{T S}=20^{\circ}, \alpha=0^{\circ}, \mathrm{AFC} @ 7 \% c_{\text {flap }}, \Delta z=1$ in......... 102

Figure 46 - Flap 40A, $C_{p}$-distributions, $\delta_{T S}=40^{\circ}, \alpha=0^{\circ}$, AFC @ 7\% $c_{\text {flap }}, \Delta z=1$ in......... 103

Figure 47 - Flap $40 \mathrm{~A}, \Delta C_{l}$ vs. $C_{\mu} \cdot\left(100-x_{a c t} / c_{f l a p}\right)^{0.5} / \delta_{T S}$ (left, $\Delta z=1$ in.) \&

$\left(0.5 C_{\mu}\right)^{0.5} \cdot C_{Q} \cdot\left(100-x_{\text {act }} / c_{\text {flap }}\right)^{0.5} / \delta_{T S}$ (right, all $\left.\Delta z\right), \alpha=0^{\circ}$.

Figure 48 - All flaps, differential lift improvements vs. $C_{\mu} \& C_{\mu f l a p}, \delta_{T S}=40^{\circ}, \Delta z=1$ in. 106 Figure 49 - Flap 60B \& 40B, best performance based on $\left(C_{d t}+C_{\mu}\right)_{\min }$ vs. $C_{l, d e s}$, best location, $\Delta z=1$ in. 108

Figure 50 - Flap 40A \& 20A, best performance based on $\left(C_{d t}+C_{\mu}\right)_{\min }$ vs. $C_{l, d e s}$, best

location, $\Delta z=1$ in.

Figure 51 - All flaps, maximum performance coefficients vs. $\Delta z, \alpha=0^{\circ}$, best actuation locations.

Figure 52 - Flap 40A, best performance based on $\left(C_{d t}+C_{\mu}\right)_{\min }$ for all $\alpha$-sweeps vs. $C_{l, d e s}$, AFC@7\% $c_{\text {flap }}, \Delta z=1$ in.

Figure 53 - Flap 40A, best performance based on $\left[\left(C_{d t}+C_{\mu}\right)_{\text {min }}\right]_{\text {safe }}$ for all $\alpha$-sweeps with $20 \%$ safety margin in $C_{l, \max }$ vs. $C_{l, d e s}$, AFC @ $7 \% c_{\text {flap }}, \Delta z=1$ in. 


\section{LIST OF FIGURES - Continued}

Figure 54 - Flap 40A, best performance based on $\left(C_{d t}+C_{E}\right)_{\min }$ for all $\alpha$-sweeps vs. $C_{l, d e s}$, AFC@ $7 \% c_{\text {flap }}, \Delta z=1$ in... 117 


\section{LIST OF TABLES}

Table 1 - Tested parameters for various flap configurations. ....................................... 32

Table 2 - Flap 20A, maximum efficiency comparison, sweeping vs. non-sweeping jets. 71 


\section{ABSTRACT}

Parameters governing separation control with sweeping jet actuators over a deflected flap are investigated experimentally on a generic "Multiple Flap Airfoil" (MFA). The model enables an extensive variation of geometric and aerodynamic parameters to aid the scaling of this novel flow control method to full-size applications.

Sweeping jets exit from discrete, millimeter-scale nozzles distributed along the span and oscillate from side-to-side. The sweeping frequency is almost linearly dependent on the supplied flowrate per actuator. The measured thrust exerted by a row of actuators agrees well with vectored momentum calculations. Frequency and thrust measurements suggest that the jet velocity is limited to subsonic speeds and that any additional increase in flowrate causes internal choking of the flow.

Neither the flowrate nor the momentum input is found to be a sole parameter governing the lift for varying distance between adjacent actuators. However, the product of the mass flow coefficient and the square root of the momentum coefficient collapses the lift onto a single curve regardless of the actuator spacing. Contrary to other actuation methods, separation control with sweeping jets does not exhibit any hysteresis with either momentum input or flap deflection. A comparison between sweeping and non-sweeping jets illustrates the superior control authority provided by sweeping jets. Surface flow visualization on the flap suggests the formation of counter-rotating pairs of streamwise vortices caused by the interaction of neighboring jets. 
The actuation intensity required to attach the flow increases with increasing downstream distance from the main element's trailing edge and increasing flap deflection. No obvious dependence of the ideal actuation location on actuator spacing, flap deflection, angle of attack, or actuation intensity is found within the tested range. Comparisons between experimental and numerical results reveal that the inviscid flow solution appears to be a suitable predictor for the effectively and efficiently obtainable lift of a given airfoil configuration. The flap size affects the achievable lift, the accompanying drag, and the required flap deflection and actuation intensity. By controlling separation, the range of achievable lift coefficients is doubled without significant penalty in drag even when considering a safety margin for the maximum applicable incidence. 


\section{NOMENCLATURE}

$$
\begin{aligned}
& A_{\text {flap }}=\text { total flap area } \\
& A_{\text {nozzle }}=\text { exit area of one nozzle } \\
& A_{\text {ref }}=\text { reference area } \\
& b \quad=\text { airfoil } / \text { wing span } \\
& c=\text { total chord length } \\
& c_{\text {flap }}=\text { flap chord length } \\
& C_{d t}=\text { total drag coefficient } \\
& C_{E} \quad=\text { input power coefficient } \\
& C_{l} \quad=\quad \text { lift coefficient } \\
& C_{l, d e s}=\text { desired lift coefficient } \\
& C_{p} \quad=\text { pressure coefficient } \\
& C_{Q}=\text { volume flowrate coefficient } \\
& C_{\mu} \quad=\text { momentum coefficient based on total chord length } \\
& C_{\mu f l a p}=\text { momentum coefficient based on flap chord length } \\
& F_{\text {calculated }}=\text { calculated total jet momentum } \\
& F_{\text {jet }}=\text { total theoretical jet momentum } \\
& F_{\text {measured }}=\text { measured jet momentum in x-direction } \\
& F M \quad=\text { figure of merit } \\
& F^{+} \quad=\text { reduced frequency } \\
& f_{\text {sweep }}=\text { sweeping frequency } \\
& J \quad=\text { total jet momentum }
\end{aligned}
$$




\section{NOMENCLATURE - Continued}

$$
\begin{array}{ll}
L & =\text { lift force } \\
L / D & =\text { lift-to-drag ratio } \\
\dot{m} & =\text { mass flowrate } \\
n & =\text { total number of actuators per row } \\
P & =\text { input power for actuation } \\
P_{\text {atm }} & =\text { atmospheric pressure } \\
P_{\text {cal. }} & =\text { calibration pressure of flow meter } \\
P_{\text {gauge }} & =\text { gauge pressure at flow meter } \\
Q_{\text {ind. }} & =\text { pressure corrected volume flowrate } \\
Q_{\text {ind }} & =\text { indicated volume flowrate on flow meter } \\
R_{\text {Re }} & =\text { Reynolds number } \\
U_{\text {inf }} & =\text { free-stream velocity } \\
x & =\text { airfoil thickness } \\
x_{\text {act }} & =\text { streamwise direction } \\
&
\end{array}
$$




\section{INTRODUCTION}

The ability to passively and actively manipulate a flow field has become one of the most researched topics in fluid mechanics since Prandtl's first boundary layer control experiments in $1904^{[1]}$. The list of possible applications for passive and active flow control is almost endless (e.g., increase in aerodynamic performance of lifting bodies, delay of laminar-to-turbulent transition, noise reduction, enhanced combustion, increase in aircraft speed, weapon applications, maneuverability of aircrafts, etc.). Especially over the last decades the need to reduce the fuel consumption and emission of greenhouse gases from commercial and military aircrafts has been driven by economic challenges and growing concerns regarding climate change. A number of approaches to reduce an aircraft's fuel consumption are being pursued including lightweight materials, design optimizations, and engine improvements, just to name a few. In particular, the control of flow separation from internal and external aerodynamic surfaces has gained significant interest as summarized by the proceedings of the biennial AIAA Flow Control Conferences which started in 2002. A multitude of passive and active means to control flow separation have been proposed and investigated. Some of the most common approaches involve vortex generators ${ }^{[2,3]}$, miniature trailing edge devices ${ }^{[4]}$, continuous and oscillatory blowing and suction ${ }^{[5-9]}$, microjets ${ }^{[10-13]}$, and zero-net-mass-flux actuation (e.g., plasma actuators ${ }^{[14]}$ and synthetic jets ${ }^{[15-23]}$ ). These methods and their feasibility for full-scale applications have been reviewed and evaluated by numerous authors ${ }^{[24-30]}$. The various methods entail different underlying mechanisms to control flow separation and therefore, they inherit specific advantages and disadvantages. For example, passive 
control devices can be effective in controlling the flow but add parasitic drag when not needed. Active control methods, such as continuous blowing and suction, can energize the boundary layer by injecting high momentum fluid into the boundary layer or removing low-momentum fluid from the boundary layer. Although these methods are effective due the significant momentum input, the benefits often do not outweigh the high flowrates required. A different approach is provided by synthetic jets which do not require any source of mass flow. The oscillatory control intends to amplify natural instabilities in the shear layer of the separated flow to enhance mixing with the outer flow region and thereby energize the boundary layer. However, limitations in momentum output, and the complexity and weight of the equipment leave the effectiveness of this method questionable for full-scale applications ${ }^{[7]}$. In order to overcome this limitation and maintain the advantageous effects of oscillatory control, oscillatory blowing (with and without suction) has been investigated and shown to be an effective means to control flow separation ${ }^{[5,6]}$. However, the required mechanisms and complex system integration remain disadvantages of this method. An alternative to controlling the spanwise vorticity, as intended by aforementioned control methods, is offered by discretely distributed, high velocity jets $^{[10-13]}$. These jets reduce the required bleed air because they not only control flow separation by the inserted momentum but mostly by enhancing the mixing with the outer flow through streamwise vortices ${ }^{[11,12]}$. Although this method has been proven to be effective in controlling separation and reducing the required flowrates, the spacing between adjacent jets remains too small in order to be efficient. Therefore, a further reduction in flowrate is limited. Consequently, an effective, but simple and practical 
actuation method involving high velocity jets at a larger spacing and the advantageous effects of an oscillatory nature is desired. These requirements are met by fluidic oscillators or so-called sweeping jet actuators.
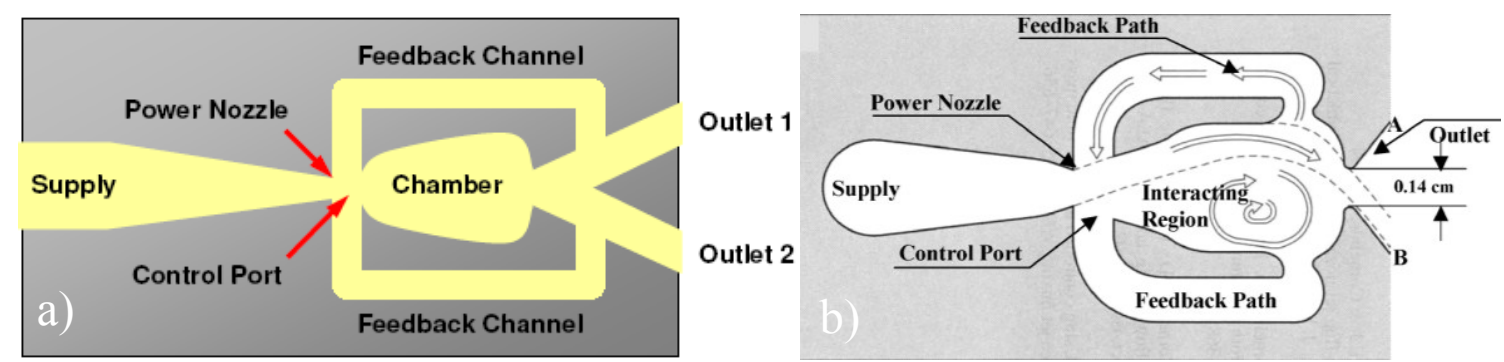

Figure 1 - Conceptual actuator designs: a) discrete jet outlets ${ }^{[40]}$, b) continuous sweeping range ${ }^{[39]}$.

Sweeping jet actuators emit a continuous but spatially oscillating jet when supplied with fluid under pressure. The oscillations do not involve any moving parts and are solely based on the internal fluid dynamics by incorporating a fluidic feedback loop into the actuator design (Figure 1). As the fluid passes through the actuator, the bistable effect of fluid entrainment from a limited space forces the jet to attach to either wall of the chamber (i.e., the Coanda effect) thereby delivering a signal through the feedback channel. The interaction at the inlet of the chamber causes the jet to separate from that side and attach to the opposite side of the chamber where the process is reinitiated. The jet's sweeping frequency can vary from the order of $10 \mathrm{~Hz}^{[31,32]}$ to the order of $20 \mathrm{kHz}^{[33]}$ depending on the actuator's geometry, size, and supplied flowrate. Although discovered more than fifty years ago at the Harry Diamond Research Laboratories, these devices have mostly been operated with liquids as a working fluid ${ }^{[34,35]}$ (e.g., windshield washers, sprinklers, shower heads, Jacuzzis, etc.). Only in recent years has this concept been applied in conjunction with active flow control (e.g., separation ${ }^{[36,37]}$, noise $\mathrm{e}^{[38,39]}$, and 
combustion control $\left.{ }^{[40]}\right)$. The characteristics of various actuator types, conceptually similar to the designs shown in Figure 1, have been investigated experimentally ${ }^{[31-33,41-48]}$ and numerically ${ }^{[48-52]}$ by numerous researchers. Several active and passive concepts to provide the oscillatory character of the emitted jet have been proposed, including selfinduced feedback ${ }^{[43]}$ and external control ${ }^{[42,44-47]}$ designs. Early actuators for flow control used a splitter in the outlet nozzle ${ }^{[36,40,55]}$, which forced the jet to exit through discrete outlets (Figure 1a) instead of having a continuous sweeping range (Figure 1b). Remarkable results on separation control ${ }^{[37,53,54,56-60]}$ were also achieved with fluidic oscillators providing continuous sweep from side to side as illustrated in Figure $1 \mathrm{~b}$ which conceptually represents the design of the actuators used in this study. They are small (order of millimeters) and driven by a high-pressure air source that enable the jet to oscillate in spanwise direction at large angles relative to the axis of the nozzle and at frequencies of the order of $\mathrm{kHz}$. Therefore, reduced frequencies of $O(10)$ and higher are achieved which circumvent the common wisdom of $F^{+}=O(1)^{[19]}$. DeSalvo et al. ${ }^{[60]}$ argue that high frequency actuation may be beneficial because it decouples the actuation method from the dominant frequencies of the separated flow. Wygnanski ${ }^{[19]}$ and Nagib et al. ${ }^{[7]}$ indicated that the ideal frequency for periodic excitation is dependent on incidence and flap deflection due to the changing size of coherent structures in the separated flow regime. Therefore, low-frequency actuation requires detailed knowledge of the natural flow patterns under various conditions which makes it a challenging task to optimize this actuation method. This difficulty may be avoided by high-frequency actuation if it can provide similar effectiveness. 
In recent years, significant aerodynamic improvements were achieved with the type of actuators shown in Figure $1 \mathrm{~b}$ by controlling flow separation over the flap of several two-dimensional (NACA $0015^{[2,53,54]}$, NACA $0021^{[56]}$, V-22 airfoil ${ }^{[37]}$, diffuser model ${ }^{[36]}$, DU96 airfoil ${ }^{[55]}$, and ADVINT airfoil ${ }^{[58-60]}$ ) and three-dimensional models (finite wing and $1 / 10^{\text {th }}$ scale, powered $\mathrm{V}-22$ model $^{[37]}$, and a $\lambda$-wing configuration ${ }^{[57]}$ ). The effectiveness of these devices is exemplified by separation control on various V-22 configurations ${ }^{[37]}$ at moderate inputs of mass and momentum. The lift-to-drag ratio of a finite wing/nacelle configuration was increased by $60 \%$ and the download-to-thrust ratio of a $1 / 10^{\text {th }}$ scale, powered V-22 model was reduced by $30 \%$ which is superior to the $17 \%$ reduction achieved with earlier actuation methods ${ }^{[23]}$.

\subsection{Research Objectives}

Although the effectiveness of sweeping jet actuators has been convincingly demonstrated, most of the parameters involved were chosen intuitively or from limited experience with other actuation methods. Therefore, this work presents a parametric study to establish parameters governing separation control over the flap of a generic "Multiple Flap Airfoil" (MFA) with sweeping jet actuators and thereby aid the scaling of these devices to full-size applications. After characterizing the properties of the particular actuators used, their performance on controlling flow separation is evaluated in conjunction with numerous parameters (e.g., flap deflection, size and geometry, incidence, actuation location, spacing between adjacent actuators, actuation intensity, sweeping frequency, free-stream velocity, etc.). Although no detailed investigation of the flow field is conducted, the underlying mechanisms responsible for the effective control 
of flow separation with sweeping jet actuators are discussed based on surface flow visualization experiments and other implicative observations. Limitations in effectively and efficiently achievable lift obtained with this actuation method are assessed by comparisons to potential flow solutions. Due to typically small levels of momentum the improvements obtained with other actuation methods are often limited by the lift expected from incidence and camber ${ }^{[7,19,27]}$. However, since sweeping jets are emitted at high velocity and frequency, supercirculation based on the jet flap principle ${ }^{[61-65]}$ may be achievable. All of the results and discussion will congregate in a proposal of how to scale and optimize this novel actuation method for specific full-size applications. Although a wide spectrum of conclusions can be extracted from this study, some questions are raised or left unanswered for future work to be conducted.

Part of the results discussed herein have been presented at two AIAA conferences in $2010^{[66]}$ and $2011^{[67]}$. 


\section{EXPERIMENTAL SET-UP AND INSTRUMENTATION}

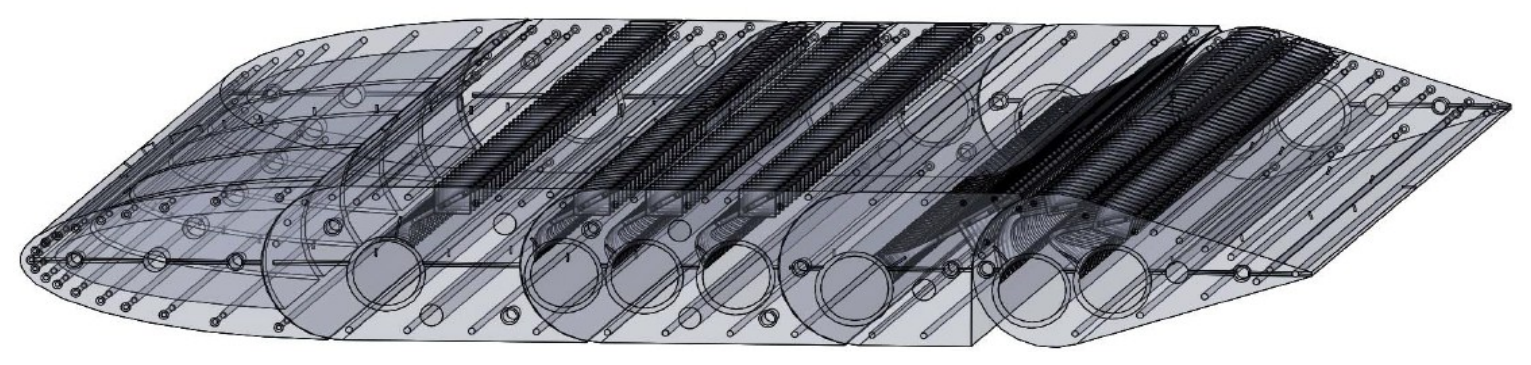

Figure 2 - Design of Multiple Flap Airfoil (MFA).

The investigation of parameters governing separation control with sweeping jet actuators requires a test bed which provides the necessary flexibility to simply change a multitude of parameters. Therefore, an airfoil made of five segments was designed (Figure 2). These segments may be combined and interchanged resulting in a $20 \%, 40 \%$, and $60 \%$ chord flap with different shapes as outlined in Figure 3. Hereafter, the various flap configurations are abbreviated according to Figure 3. The symmetric airfoil spans 24in. and has a chord length of $12 \mathrm{in}$. with a maximum thickness of $12.5 \% \mathrm{c}$. The nose segment has a $4: 1$ ratio elliptical shape providing $25 \%$ of the total chord length. Two mid-segments, each having a chord length of $20 \% c$, maintain a constant thickness of 1.5in. A shorter, $15 \% c$ segment is always placed upstream of the flap to provide actuation from the flap shoulder. The trailing edge flap segment also provides $20 \%$ of the total chord length, tangentially and linearly converging to a sharp trailing edge stemming from the desire to obtain an ideal Kutta condition. The same simple flap geometry was tested in configuration 40A (Figure 3) with a single flap segment providing $40 \% \mathrm{c}$. The cambered flap configurations shown in Figure 3 originated as a consequence of initial tests with configuration $60 \mathrm{~A}$. Although the flap is symmetric, the rapid convergence of 
the last segment toward the sharp trailing edge deflects the flow by $17^{\circ}$ providing a significant pressure gradient which causes the flow to separate at small flap deflections. Therefore, the effectiveness of a certain actuation location and intensity is governed by achieving flow attachment not just over the main part of the flap but also over the converging trailing edge segment. In order to decouple these effects the set-up was changed to an inflected, cambered flap possessing a straight top surface by rotating the trailing edge segment to the proper position (Figure 3, configuration 60B and 40B). Although the flap's camber affects the baseline lift and drag compared to the symmetric flap configuration, the parameters governing actuation are identified more explicitly when actuation controls the flow over a straight surface as will be verified by configuration $40 \mathrm{~A}$ and $20 \mathrm{~A}$.

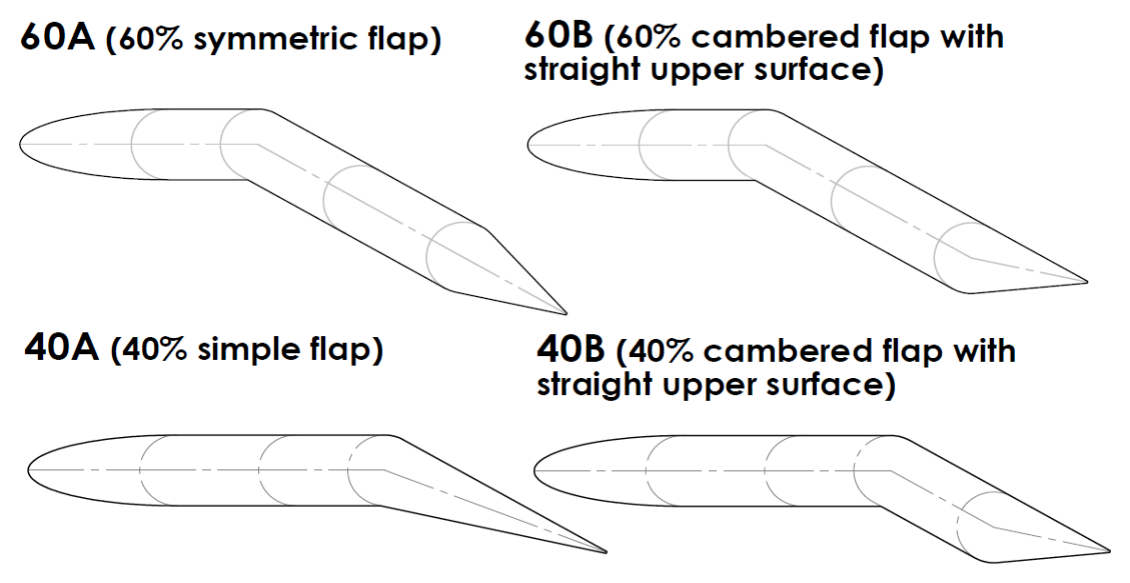

$20 A(20 \%$ simple flap)

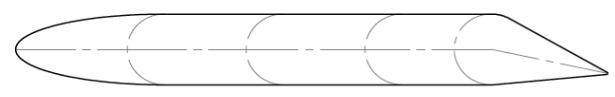

Figure 3 - Various flap sizes and shapes tested with MFA model.

In order to simplify the discussion of flap deflection and various flap sizes and geometries, the flap's deflection is referred to as $\delta_{T S}$ which defines the divergence angle 
that the flow experiences over the flap's top surface (i.e., the angle between the main element's top surface and the flap's top surface). The common notation of $\delta_{f}$ remains the usual definition of flap deflection as the angle between the main element's chord line and the flap's chord line. Note that $\delta_{f}$ and $\delta_{T S}$ are only shifted by a constant value which depends on the flap's geometry.

Due to the complicated internal structures of the design (Figure 2) all segments of the model were manufactured with "High Definition Stereo Lithography (HDSL)" which grew the parts in layers of 0.004in., thus providing adequate accuracy for the internal and external geometries. A total of seven rows of sweeping jet actuators are embedded inside the airfoil's segments. Each row contains 47 actuators distributed over the entire span at an even spacing of $\Delta z=0.5 \mathrm{in}$. The emitted jets are inclined at $30^{\circ}$ to the airfoil's local surface. The chosen locations for these rows permit separate or combined actuation from various locations for all flap configurations. For each row of actuators a perforated brass tube feeds compressed air to the individual actuators. A second brass tube that fits snugly into the outer one in a telescopic arrangement has three rows of holes with a spacing of $0.5 \mathrm{in} .$, 1in., and 1.5in. depending on its azimuth. By rotating the inner tube one may selectively pressurize only every second or third actuator in that row. Leakage from inactive actuators was prevented by plugging the actuators' outlet with soft, removable material. Air supply tubes to inactive locations were sealed to avoid any entrainment of air outside the test section. The air supply is controlled by a pressure regulator and monitored by a visual scale Brooks Instrument flow meter coupled with a pressure gauge to correct the indicated air flow measurement for pressure and thereby density. The 
correction formula was provided by the manufacturer of the flow meter (Equation (1), $\left.P_{\text {cal }}=14.7 \mathrm{psi}\right)$.

$$
Q=Q_{\text {ind. }} \cdot \sqrt{\frac{P_{\text {gauge }}+P_{\text {atm }}}{P_{c a l}}}
$$

The segments of the model are held in place on both sides by endplates made out of Plexiglas. Figure 4 illustrates the complete set-up of the model for the $20 \%$ flap configuration. Dowel pins fix the location of the segments to one another while threaded rods provide stability to the model. All transition areas between the segments of the airfoil and the inactive rows of actuators are covered with thin tape to provide a smooth and continuous surface. Approximately 2in. inward from the endplates boundary layer fences made out of $0.015 \mathrm{in}$. thick sheet metal are installed (Figure 4) to minimize the effects associated with the interaction between the airfoil and the test section's side walls which could reduce the area of two-dimensionality. The boundary layer fences are not mounted onto the model directly. Instead, they are connected to the endplates by long machine screws which allow for a continuous adjustment of the distance to the endplates (Figure 4a). The main element and the flap have separate, overlapping fences to allow a decoupled movement of the flap which is mounted onto rotating inserts that fit precisely through cut-outs in the endplates. The flaps can be deflected continuously from $0^{\circ}$ to $90^{\circ}$, therefore providing the possibility for download alleviation experiments relevant to a tilt rotor airplane in hover ${ }^{[37]}$. The variation of incidence of the model's main element is automated by a turn table being connected to a stepper motor (Figure 4b). Based on the 
same design concept, all flap configurations have a different set of endplates, rotation pieces, and boundary layer fences, all manufactured on a CNC machine.
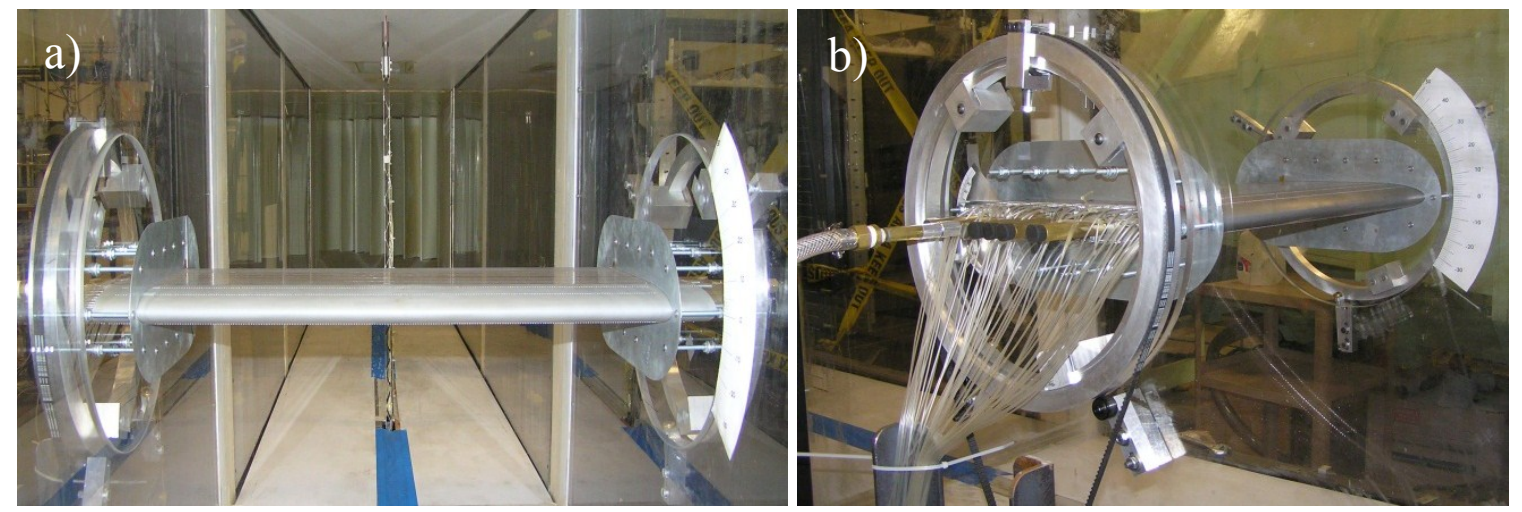

Figure 4 - Complete assembly of MFA in $20 \%$ flap configuration.

The model's endplates are mounted flush with the sidewalls of a closed-loop wind tunnel test section which is $2 \mathrm{ft}$ wide and $3 \mathrm{ft}$ high. The wind tunnel is located in the Aerolab at the University of Arizona, Tucson, AZ. The floor and ceiling of the test section are slightly diverging to account for boundary layer growth. The maximum freestream velocity is $30 \mathrm{~m} / \mathrm{s}$ yielding a maximum Reynolds number of 600,000 based on the model's chord length. To minimize Reynolds number and transition effects trip-strips are applied to the airfoil's top and bottom surface at $x / c=9 \%$ and $x / c=0.18 \%$.

Sixty-eight pressure taps are distributed in a staggered array along the airfoil's mid-span yielding the mean pressure distribution over the model's surface. Channels running underneath the surface along the entire span of the airfoil convey the pressure signal to pressure sensors. These channels are sealed on one side whereas stainless steel tubes are attached to the other side leading the signal through plastic tubing to measurement equipment located outside the test section (Figure 4b). A rake with 26 Pitot tubes measures the model's total drag by assessing the momentum deficit in its wake. 
The rake is mounted on a traversing mechanism which allows increasing the resolution of the wake measurements as needed. Two static pressure tubes located on each side of the rake enables the assessment of the static pressure variations across the wake in order to correct the drag calculation. The rake's distance from the airfoil is continuously adjustable; however, it was fixed at three chord lengths downstream of the trailing edge of the model for the majority of the experiments. All pressure measurements were conducted with a PSI ${ }^{\mathrm{TM}} 780 \mathrm{~B}$ pressure measurement system which provides an accuracy of $\pm 3 \mathrm{~Pa}$ corresponding to $0.12 \%$ of the full scale. The data acquisition and the movement of the wake rake and turn table are automated and controlled by a LabVIEW ${ }^{\mathrm{TM}}$ program. Due to the oscillatory nature of the sweeping jets, concerns regarding fluctuations in the pressure measurements were alleviated by incorporating a convergence criterion into the $\mathrm{LabVIEW}^{\mathrm{TM}}$ program. This ensured convergence of the mean pressure at every port with a relative error of less than $1 \%$.

Besides pressure measurements, this study was complimented by surface flow visualization experiments, and hotwire and thrust measurements. Surface flow visualization experiments were conducted with China Clay applied over the top surface of the flap. To avoid impregnations from inactive rows of actuators and transition seams between the individual airfoil segments a thin sheet metal plate (0.010in. thick) was attached over the flap downstream of the desired actuation location and then laminated with black foil. China Clay is a mixture of titanium white oil color and a quickly evaporating solvent (e.g., kerosene or lighter fluid). Pictures were taken once the China Clay had dried. Bench-top experiments were conducted to characterize the properties of 
the sweeping jet actuators. Hotwire measurements with an A.A. Lab Systems LTD. hotwire anemometer yielded the time-resolved oscillatory behavior of the jets and therefore, the sweeping frequency. For measurements of the thrust exerted by a row of actuators the model was mounted on a knife edge. An Interface ${ }^{\mathrm{TM}}$ load cell $(<0.18 \%$ full scale error) measured the forces in $x$-direction in absence of a free-stream. Both, the hotwire and load cell, data were recorded by a LabVIEW ${ }^{\mathrm{TM}}$ program. Additional postprocessing and visualization of most of the experimental data were exercised with Matlab $^{\mathrm{TM}}$. 


\section{PARAMETER OVERVIEW AND DISCUSSION}

Before entering the discussion of results this chapter provides an overview of the parameters involved in separation control with sweeping jet actuators. Similar to traditional active flow control (AFC) methods, a large number of parameters governs the aerodynamic characteristics of a particular lifting body. Equation (2) lists the most salient parameters governing the lift and divides them into model specific and AFC specific parameters.

$$
L=L\left(\begin{array}{c}
\underbrace{}_{\text {inf }}, \rho_{\text {inf }}, c, c_{\text {flap }}, b, \text { camber }, t, \alpha, \delta_{T S}, x_{\text {sep }} \\
\underbrace{x_{\text {act }} J, Q, U_{\text {jet }}, A_{\text {nozzle }}, \rho_{\text {jet }}, \Delta z, n, \alpha_{\text {jet }}, f_{\text {sweep }}, \theta_{\text {sweep }}}_{\text {model specific }}
\end{array}\right)
$$

This list is certainly not complete and does not account for certain factors (e.g., finite wing configurations). Note that the list also does not include any variation of the actuators' design which would exponentially enlarge the parameter space even further. The actuators' geometry has been left unchanged in this study. The parameter space exposed in Equation (2) is difficult to fully explore experimentally. Therefore, attempts need to be made to reduce the list of parameters. Certain simplification and assumptions can be introduced which make some of the listed parameters redundant. For example, if the actuators are distributed evenly along the span, the number of actuators is fully defined by the span and spacing. Also, if all actuators are the same size and equally supplied with air, the jet velocity and momentum can be excluded from the list. However, these simplifying assumptions may not be optimal for three-dimensional configurations 
(e.g., tapered wing) where the spacing and size of the actuators may change along the span for optimal performance.

For a reasonable experimental investigation the list of parameters needs to be reduced even further by fixing the value of some parameters for this study. For example, the exit angle at which the jets are emitted to the local surface was fixed to $30^{\circ}$ which agrees well with the coarsely optimized value determined by DeSalvo et al. ${ }^{[58]}$. This value was chosen based on experience with other actuation methods and in order to simplify the geometric integration of the actuators into the model. However, later studies by DeSalvo et al ${ }^{[59,60]}$ propose an almost tangential exit angle to be ideal which, due to geometric effects, is not entirely conclusive. For the range of relatively small exit angles the emitted jets are expected to attach to the surface immediately downstream of the exit ${ }^{[68]}$ (see Chapter 4.5). Therefore, the actuators' performance may not depend significantly on the exit angle as indicated by DeSalvo et al. ${ }^{[58]}$. However, studies of microjets ${ }^{[1-13]}$ suggest much larger exit angles around $90^{\circ}$, thereby penetrating further away from the model's surface and affecting the ideal actuation location. Although the investigation of the exit angle is a necessary component of understanding and optimizing separation control with sweeping jets, it is left to future studies with a more suitable experimental setup.

The experimental test matrix is mainly controlled by the range and increments of parameters tested. Table 1 provides an overview of test cases performed. The choice of particular parameter values is controlled by the experimental setup and further guided by the obtained results. Flow visualization experiments were conducted for selected cases of 
interest. The comparison between sweeping and non-sweeping jets is based on the $20 \%$ flap configuration.

Table 1 - Tested parameters for various flap configurations.

\begin{tabular}{|c|c|c|c|c|c|}
\hline FLAP CONFIGURATION & $60 \mathrm{~A}$ & $60 \mathrm{~B}$ & $40 \mathrm{~A}$ & $40 \mathrm{~B}$ & $20 \mathrm{~A}$ \\
\hline \multicolumn{6}{|l|}{ PARAMETERS TESTED (symbol, unit) } \\
\hline free stream velocity $\left(U_{i n f}, \mathrm{~m} / \mathrm{s}\right)$ & \multicolumn{2}{|c|}{20,30} & \multicolumn{3}{|c|}{30} \\
\hline Reynolds number $(\operatorname{Re},-)$ & \multicolumn{2}{|c|}{$4 \cdot 10^{5}, 6 \cdot 10^{5}$} & \multicolumn{3}{|c|}{$6 \cdot 10^{5}$} \\
\hline total chord length $(c$, in.) & \multicolumn{5}{|c|}{12} \\
\hline flap chord length $\left(c_{\text {flap }} / c, \%\right)$ & 60 & \begin{tabular}{|l|l}
60 \\
\end{tabular} & 40 & 40 & 20 \\
\hline flap angle $\left(\delta_{f}, \operatorname{deg}\right)$ & $10,20,30,40$ & $\begin{array}{c}10,20,25,30 \\
35,40,45\end{array}$ & \begin{tabular}{|l|}
$0,10,20,25$ \\
$30,35,40,45$
\end{tabular} & $\begin{array}{l}0,10,20,25 \\
30,35,40,45\end{array}$ & $\begin{array}{l}20,30,35 \\
40,45,50\end{array}$ \\
\hline angle of attack $(\alpha$, deg $)$ & 0 & \begin{tabular}{|l|l}
0 \\
\end{tabular} & \multicolumn{3}{|c|}{$\alpha$-sweep for $\Delta z=1$ in., $\left(\alpha=0^{\circ}\right.$ otherwise $)$} \\
\hline actuation location $\left(x_{a c t} / c_{\text {flap }}, \%\right)$ & $0,18,36$ & $0,3,12,18,36$ & $0,7,18$ & $0,4,18,36$ & $0,18,36$ \\
\hline jets' exit angle to local surface $\left(\alpha_{\text {jet }}\right.$, deg $)$ & \multicolumn{5}{|c|}{30} \\
\hline actuator spacing $(\Delta z$, in.) & \multicolumn{5}{|c|}{$0.5,1.0,1.5$} \\
\hline momentum coefficient $\left(C_{\mu}, \%\right)$ & \multicolumn{5}{|c|}{ 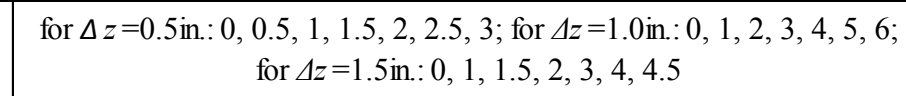 } \\
\hline
\end{tabular}

As depicted in Table 1 a further reduction in the parameter space is offered by dimensional analysis yielding some conventional non-dimensional coefficients (e.g., Reynolds number, $R e$, flowrate coefficient, $C_{Q}$, and momentum coefficient, $\left.C_{\mu}\right)$. The traditional definitions of $\operatorname{Re}$ and $C_{Q}$ are provided by Equations (3) and (4), respectively. The effect of Reynolds number is discussed in Chapter 4.2.

$$
\begin{gathered}
R e=\frac{U_{i n f} \cdot c}{v} \\
C_{Q}=\frac{Q}{A_{\text {ref }} U_{\text {inf }}}=\frac{Q}{b \cdot c \cdot U_{\text {inf }}}
\end{gathered}
$$

The definition of $C_{\mu}$ differs from its common appearance due to the discrete distribution of momentum. Equations (5)-(7) reveal the multitude of parameters involved. Equation (5) illustrates the general definition of $C_{\mu}$ without any simplifying assumptions. 
Note that the total chord length is considered for the reference area. However, the flap chord, $c_{\text {flap }}$, may be used instead to yield $C_{\mu \text { flap }}$ as discussed in Chapter 6.3.

$$
C_{\mu}=\frac{J}{1 / 2 A_{r e f} \rho_{i n f} U_{i n f}^{2}}=2 \frac{\dot{m} U_{j e t}}{b c \rho_{\text {inf }} U_{\text {inf }}^{2}}=2 \frac{\rho_{\text {jet }} Q U_{j e t}}{b c \rho_{\text {inf }} U_{\text {inf }}^{2}}
$$

In order to arrive at Equations (6) and (7), the assumption is made that the jet density at the exit is equal to the free-stream density. This assumption was also applied to Equation (4). The validity of this assumption is argued in Chapter 4.1. Additionally, the fact that the actuators (with constant exit area) are evenly spaced along the span is represented by $b=n \cdot \Delta z$. By expressing the momentum coefficient in terms of jet velocity (Equation (6)) or total flowrate (Equation (7)) the dependence on various parameters is illustrated. Equation (6) reveals that if the jet velocity were to be fixed (see Chapter 4.1) $C_{\mu}$ increases linearly with increasing actuator size and decreasing actuator spacing.

$$
C_{\mu}=2 \frac{n A_{\text {nozzle }} U_{j e t} U_{j e t}}{b c U_{\text {inf }}^{2}}=2 \frac{b / \Delta z A_{\text {nozzle }} U_{j e t}^{2}}{b c U_{\text {inf }}^{2}}=2 \frac{A_{\text {nozzle }}}{\Delta z c}\left(\frac{U_{j e t}}{U_{\text {inf }}}\right)^{2}
$$

Instead of considering a fixed jet velocity, a fixed total flowrate into the system may be considered (Equation (7)) which indicates that $C_{\mu}$ is proportional to $\Delta z$. Therefore, $C_{\mu}$ may be increased by enlarging the spacing between adjacent actuators even if the total flowrate remains constant. Throughout this study, values for $C_{\mu}$ (Table 1 ) appear larger compared to other AFC methods. However, from an application point of view, these values should be examined in terms of required flowrate. For example, considering continuous blowing through a continuous slot with the same height as the actuators used in this study, the value of $C_{\mu}$ for continuous blowing would be 20 times 
smaller if the same total flowrate were considered with sweeping jet actuators spaced at $\Delta z=1$ in. If the same momentum input is desired, continuous blowing would require $\sim 4.5$ times the total flowrate.

$$
C_{\mu}=2 \frac{Q^{Q} / n \cdot A_{\text {nozzle }}}{b c U_{\text {inf }}^{2}}=2 \frac{Q^{2}}{A_{\text {nozzle }} b / \Delta z S c U_{\text {inf }}^{2}}=2 \frac{\Delta z}{A_{\text {nozzle }} b^{2} c}\left(\frac{Q}{U_{\text {inf }}}\right)^{2}
$$

The performed test cases and the geometric properties of the model offer the investigation of parameters listed in Equation (8) either individually or combinations of them. Clearly not all of the listed parameters are independent. However, this list is intended as a summary of parameters and coefficients addressed in this study. Although the parameter space has been reduced, it allows for a comprehensive study of the dependence of the lift coefficient (and in part of the total drag coefficient) on the individual parameters and their relative significance.

$$
C_{l}=C_{l}\left(R e, \frac{c_{\text {flap }}}{c}, \alpha, \delta_{T S}, \frac{x_{\text {act }}}{c_{\text {flap }}}, C_{Q}, C_{\mu}, C_{\mu, \text { flap }}, \frac{U_{\text {jet }}}{U_{\text {inf }}}, f_{\text {sweep }}\right)
$$

The amount of test cases and parameters present the challenge of evaluating the data in a structured and organized manner. Based on the intended use of this actuation method a practical approach has been chosen. From the application point of view the subsequent chapters address four major questions:

1. How should actuation (with sweeping jet actuators) be applied?

2. Where should actuation (with sweeping jet actuators) be applied?

3. On what airfoil configuration should actuation (with sweeping jet actuators) be applied?

4. How can actuation (with sweeping jet actuators) be scaled? 
Some answers to these questions overlap due to the effect of the parameters on each other. However, they contribute to answering one encompassing question: How can a required lift be obtained most effectively and efficiently through the use of sweeping jets?

Since a desired lift may be achieved at various geometry and actuation configurations, some measure of efficiency has to be established. Therefore, the total drag at a specific lift is evaluated to yield the highest possible lift-to-drag ratio. The total drag is corrected for the expense of actuation in two ways. The first is by adding the inserted momentum which is assumed to be fully recovered in thrust. This common way of correcting the total drag yields a corrected lift-to-drag ratio which is referred to as the momentum efficiency, $\eta_{C_{\mu}}$ following the notation introduced by Seifert et al. ${ }^{[16]}$ (Equation (9)).

$$
\eta_{C_{\mu}}=\frac{C_{l}}{C_{d t}+C_{\mu}}
$$

The second way to account for the expense of actuation also follows the suggestions by Seifert et al. ${ }^{[16]}$ who introduced an input power coefficient, $C_{E}$, for the use of piezoelectric actuators. The power applied with sweeping jets is obtained from the pressure difference between supply and actuator outlet. Utilizing the previous definition and assumptions for $C_{\mu}$ yields the power coefficient in Equation (10). The power coefficient is added to the total drag assuming that if the energy expensed for the actuation system were to be used for an aircraft's engine, it would convert this energy fully into thrust. Of course this assumption is unrealistic; however, it provides an extreme 
case in evaluating the efficiency of actuation. The drag corrected by the power coefficient yields the power efficiency, $\eta_{C_{E}}$ (Equation (11)).

$$
\begin{gathered}
C_{E}=\frac{P}{1 / 2 A_{\text {ref }} \rho_{\text {inf }} U_{\text {inf }}^{3}}=\frac{\left(1 / 2 \rho_{\text {jet }} U_{j e t}^{2}\right) n A_{\text {nozzle }} U_{j e t}}{1 / 2 A_{\text {ref }} \rho_{\text {inf }} U_{\text {inf }}^{3}}=\frac{C_{\mu}}{2} \cdot \frac{U_{j e t}}{U_{\text {inf }}} \\
\eta_{C_{E}}=\frac{C_{l}}{C_{d t}+C_{E}}=\frac{C_{l}}{C_{d t}+\frac{C_{\mu}}{2} \frac{U_{j e t}}{U_{\text {inf }}}}
\end{gathered}
$$

Seifert et al. ${ }^{[16]}$ also defined a figure of merit which references the power efficiency (i.e., the corrected lift-to-drag ratio) to the baseline $L / D$ at the same geometric configuration. This measure is not discussed in this form because it is misleading. The desired lift may be achieved most efficiently (i.e., at the smallest corrected drag) at a different geometric configuration (e.g., flap deflection, incidence, and flap size). This measure is only used as a single, global quantity comparing the highest achievable $L / D$ with actuation and without actuation (Equation (12)).

$$
F M_{\max }=\frac{\eta_{\max }}{\left(\left(C_{l} / C_{d t}\right)_{\text {baseline }}\right)_{\max }}
$$

The two efficiencies addressed in this study certainly do not provide a complete evaluation of efficiency for the use of sweeping jet actuators. Many unfavorable factors, such as air supply efficiency, weight of the AFC system, and system integration and maintenance, are not included in this discussion. However, many favorable consequences of AFC, such as reduced wing size and subsequent reduction in weight, drag, and fuel 
consumption over an entire flight, are also not accounted for. A complete evaluation of efficiency will be necessary for the study of a specific application.

Although the specific geometry of the MFA may not be representative for other models, the observed trends and proposed coefficients may provide a guideline to the optimization and scaling of sweeping jet actuators for specific applications. 


\section{HOW SHOULD ACTUATION BE APPLIED?}

The first question to be answered is undoubtedly one of the most complex because it requires a detailed understanding of the properties of sweeping jet actuators and their role in separation control. First, the characteristics of sweeping jets in absence of a freestream are discussed based on hotwire and force measurements. Then the effects of Reynolds number and hysteresis are evaluated before the quantification of actuation intensity is addressed for changing actuator spacing. Unfortunately, no quantitative results for the underlying mechanisms of how sweeping jets provide the effective separation control are available to this date. However, qualitative results obtained from surface flow visualization and other indicative observations provide some initial insight. The last subsection of this chapter compares sweeping and non-sweeping jets as a failure mode example and to illustrate the benefits of the jets' sweeping motion.

\subsection{Characterization of Actuators}

The properties of similar sweeping jet actuators have been thoroughly investigated by Gregory et al. ${ }^{[33]}$. However, most of their efforts were directed at time series analyses through various experimental methods. Conversely, the current study focuses on the resolution of sweeping frequency, $f_{\text {sweep }}$, as a function of jet velocity, $U_{j e t}$, and on the thrust exerted by an array of these actuators.

Figure 5 illustrates an exemplary time series and corresponding spectrum recorded at an actuator's exit by a hotwire for a mid-range flowrate. The time series reveals the expected sinusoidal behavior of the sweeping motion which allows a distinctive definition of $f_{\text {sweep }}$. All actuators on all rows were verified to have similar oscillatory 
behavior. Based on the sweeping frequency the rows of actuators were also checked for flowrate uniformity.
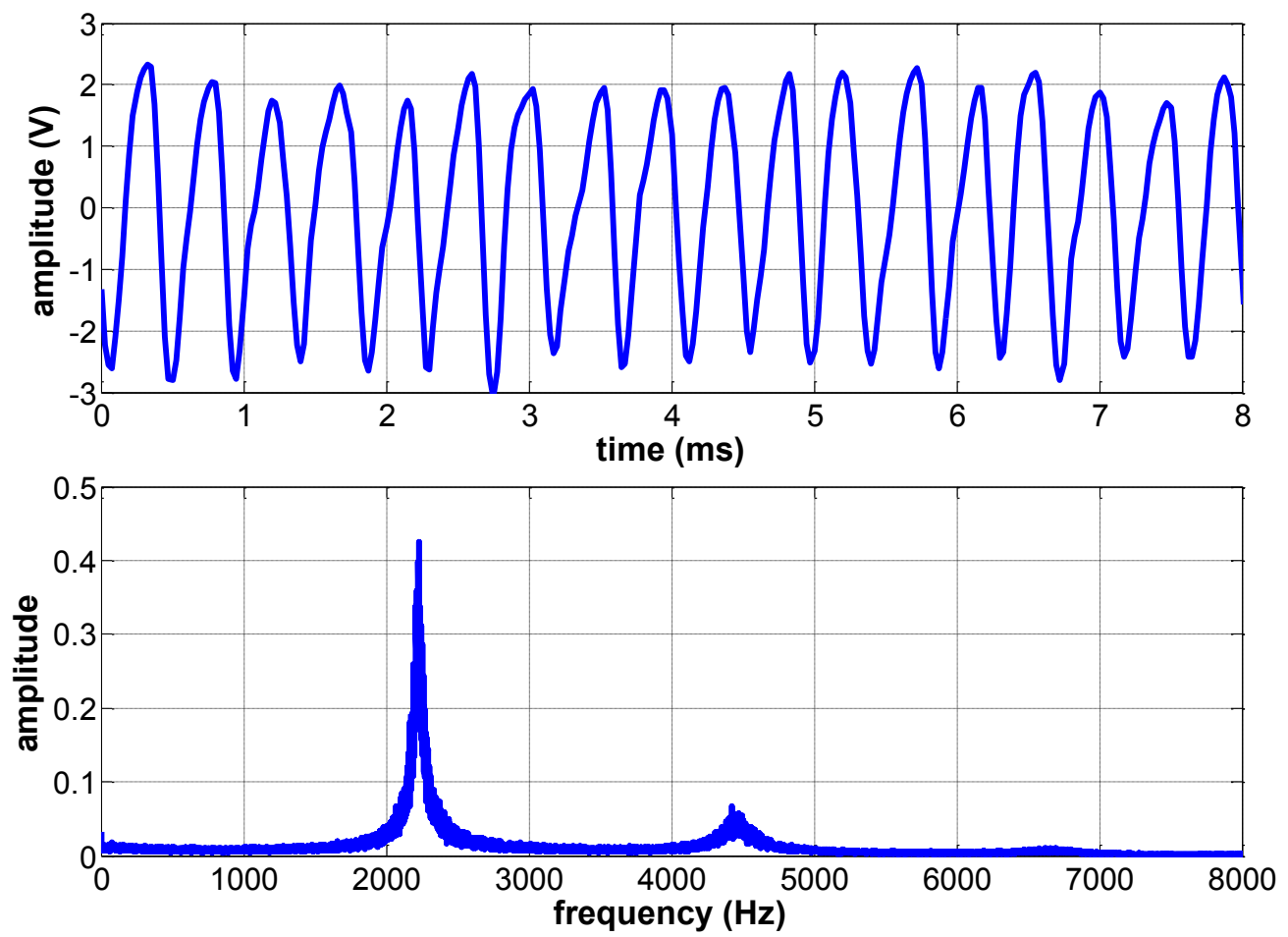

Figure 5 - Time series (top) \& spectrum (bottom) of exemplary sweeping jet actuator, $f_{\text {sweep }}=2.2 \mathrm{kHz}$.

Hotwire measurements were conducted for a wide range of flowrates and for different spacing between adjacent actuators to yield the sweeping frequency as a function of flowrate per actuator (Figure 6). The corrected flowrate obtained from Equation (1) is used to calculate the jet velocity at the exit. The measurement of exit velocity is difficult with a hotwire, because the high velocities require the necessary equipment to calibrate the hotwire for these speeds. Also, any measurements in close proximity to the exit are very sensitive to the proper positioning of a probe especially if the probe dimension is comparable to the actuator outlet dimension. Note that with increasing actuator spacing the supply pressure increases and therefore, the same 
corrected volume flowrate per actuator is achieved at different pairs of uncorrected volume flowrates and supply pressures. The almost perfect agreement between the various actuator spacings over the entire range of flowrates (Figure 6) proves the validity of Equation (1).

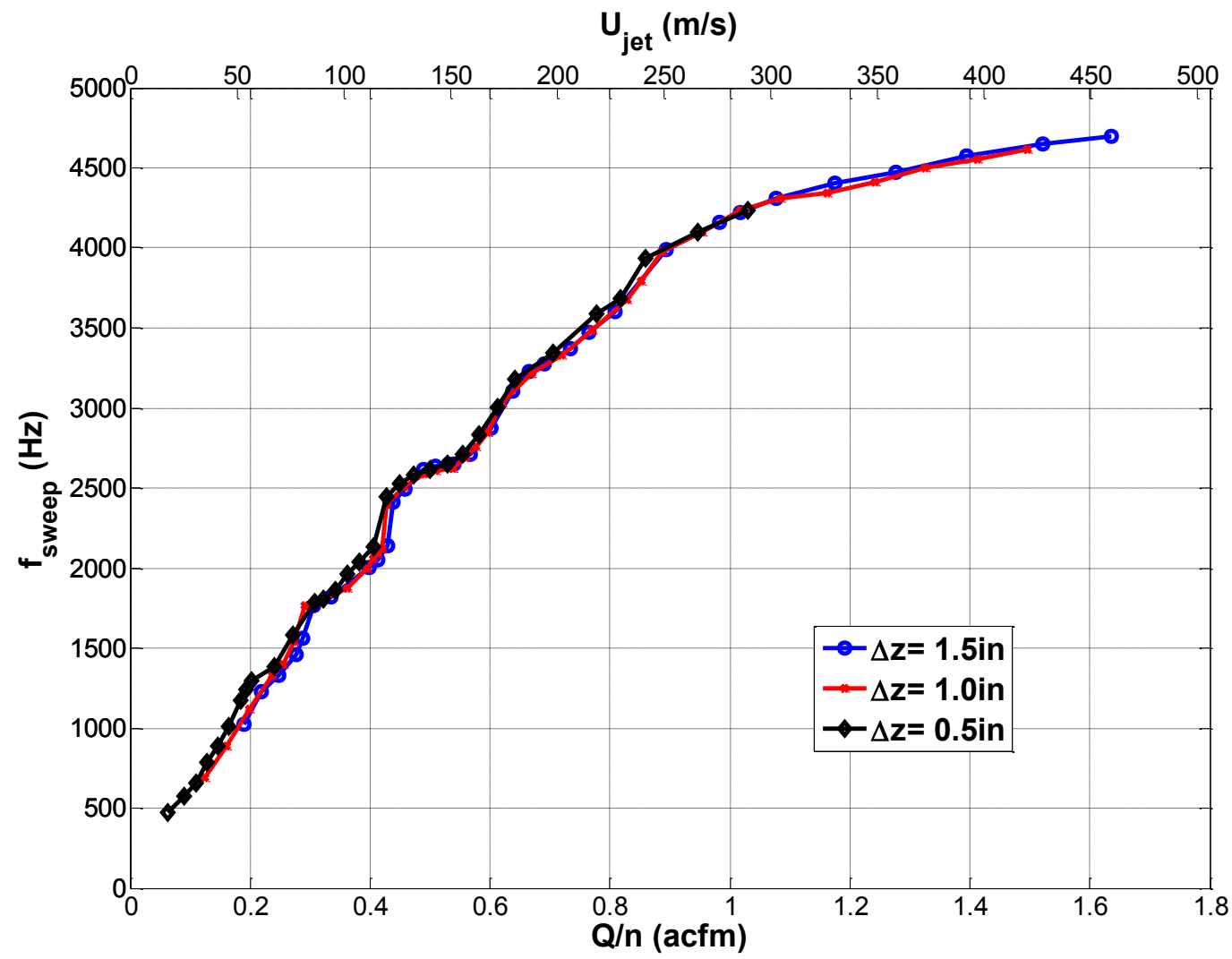

Figure 6 - Sweeping frequency vs. volume flowrate per actuator for various $\Delta z$.

The results shown in Figure 6 indicate that the sweeping frequency is solely a function of volumetric flowrate per actuator independent of the inlet conditions. For reference purposes the calculated jet velocities based on the corrected flowrate are plotted on a second $x$-axis implying that for subsonic jet velocities up to $U_{j e t} \approx 250 \mathrm{~m} / \mathrm{s}$ the sweeping frequency is almost linearly proportional to the flowrate and hence, to the jets' exit velocity. However, as the exiting jets are approaching sonic speed the frequency 
saturates close to $5 \mathrm{kHz}$ implying that the maximum exit velocity is limited to subsonic speed. A further increase in flowrate may only cause internal choking and increase in density of the emitted jet without further increase in $U_{j e t}$. Although numerical studies ${ }^{[51]}$ suggest that sweeping jets may be emitted at supersonic speed, it is yet to be verified experimentally with a similar actuator geometry. On a larger scale, single actuator Raman et al. ${ }^{[41]}$ achieved supersonic jet velocities which were calculated based on the pressure ratio assuming isentropic expansion. No precise measurements of the Mach numbers were conducted. They also noted a saturation of the sweeping frequency towards higher pressure ratios and the oscillations eventually ceased due to internal shocks. External shocks were also observed. Experimental work with micro jets also claim supersonic jet velocities ${ }^{[11-13]}$. However, the advantages or disadvantages of supersonic exit velocities have not been evaluated yet. Supersonic jets may not be desirable because shocks (internal and external) may add to the overall drag and reduce the overall efficiency of the AFC system.

The saturation of the sweeping frequency when approaching sonic speed also implies that the signal delivered through the feedback channels of the actuator is dependent on the internal speed. Therefore, one may conclude that a stream through the feedback channels causes the jet to switch and not just a pressure signal which would travel at the speed of sound independent of the provided flowrate. Flow visualization experiments inside a large-scale actuator support the argument that a stream is flowing through the feedback channels ${ }^{[69]}$. Guyot et al. ${ }^{[40]}$ demonstrated that the sweeping frequency of these fluidic devices, when supplied with different fluids, is a function of 
volume flowrate and therefore jet velocity rather than mass flowrate. Although for subsonic jet velocities the sweeping frequency appears to be a linear function of flowrate per actuator, there are distinctive deviations from this linear pattern. Internal transition from laminar to turbulent flow, as suggested by Gregory et al. ${ }^{[33]}$, does not provide a reasonable explanation because the jet Reynolds numbers at which these deviations occur are well beyond the conventional transition Reynolds numbers for jet flow. Since these deviations are consistent for different actuators and at different $\Delta z$ (and therefore supply pressure) it is ensured that no measurement error is the cause for these deviations. No reasonable explanation for these phenomena has been established yet.

Simultaneous hotwire measurements at the exit of adjacent actuators connected to the same pressure source do not suggest any communication or phase lock between them. This is supported by spectral analysis of instantaneous force measurements which do not indicate any dominant frequency. Although numerically an array of actuators can produce jets sweeping in phase ${ }^{[52]}$, any disturbance in the start-up process of the sweeping motion or in the actuators' geometry yields jets sweeping out of phase without recovery. Experimentally (and probably numerically), it is possible to produce jets sweeping in phase or shifted by $180^{\circ}$ by interconnecting the feedback channels of adjacent actuators $^{[69]}$. However, the effects of sweeping phase on separation control efficacy have not been investigated yet.

In order to assess the total momentum applied by a row of actuators in $x$-direction, the model was mounted on a knife edge and the applied force in $x$-direction recorded by a load cell. To avoid surface effects such as jet deflection or surface friction the entire flap 
was removed from the model allowing the sweeping jets to emanate from the flap shoulder into unobstructed space aligned with the load cell axis. Figure 7 presents the ratio between the measured and calculated forces as a function of flowrate per actuator for various $\Delta z$. The calculated jet velocity is shown for reference purposes. The agreement between the different actuator spacings is within $10 \%$ for $U_{j e t}<130 \mathrm{~m} / \mathrm{s}$ and even those differences decrease at higher flowrates. The ratio between measured and calculated jet force remains almost constant over the entire range of tested flowrates thereby supporting the validity of the momentum calculations. However, as the jets' velocity approaches sonic speed the force ratio drops indicating the onset of compressibility effects which reduce the validity of the momentum calculations.

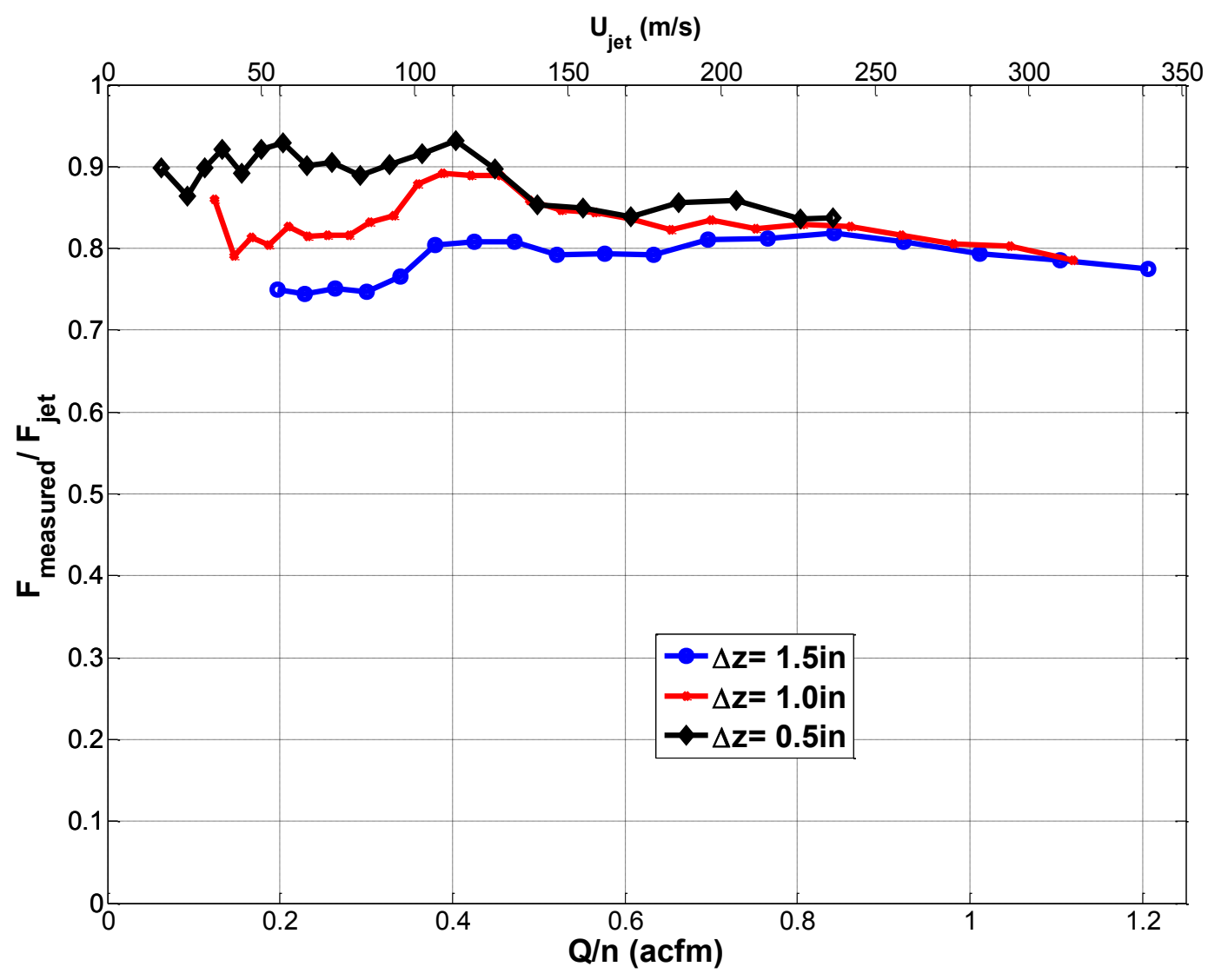

Figure 7 - Ratio of measured \& calculated jet momentum vs. flowrate per actuator. 
As the spacing between adjacent actuators increases, the measured jet momentum becomes smaller. This is especially true for smaller flowrates, implying that a possible interaction between neighboring jets decreases the maximum angle of sweep and thereby bounding more momentum in $x$-direction. The average measured jet momentum in $x$ direction is expected to be less than the calculated jet momentum due to the excursions of the sweeping jets away from the $x$-direction. Therefore, the measured momentum should be directly dependent on the sweeping angle, $\theta_{\text {sweep }}$, of the jet which may be approximated by a simplified mathematical model.
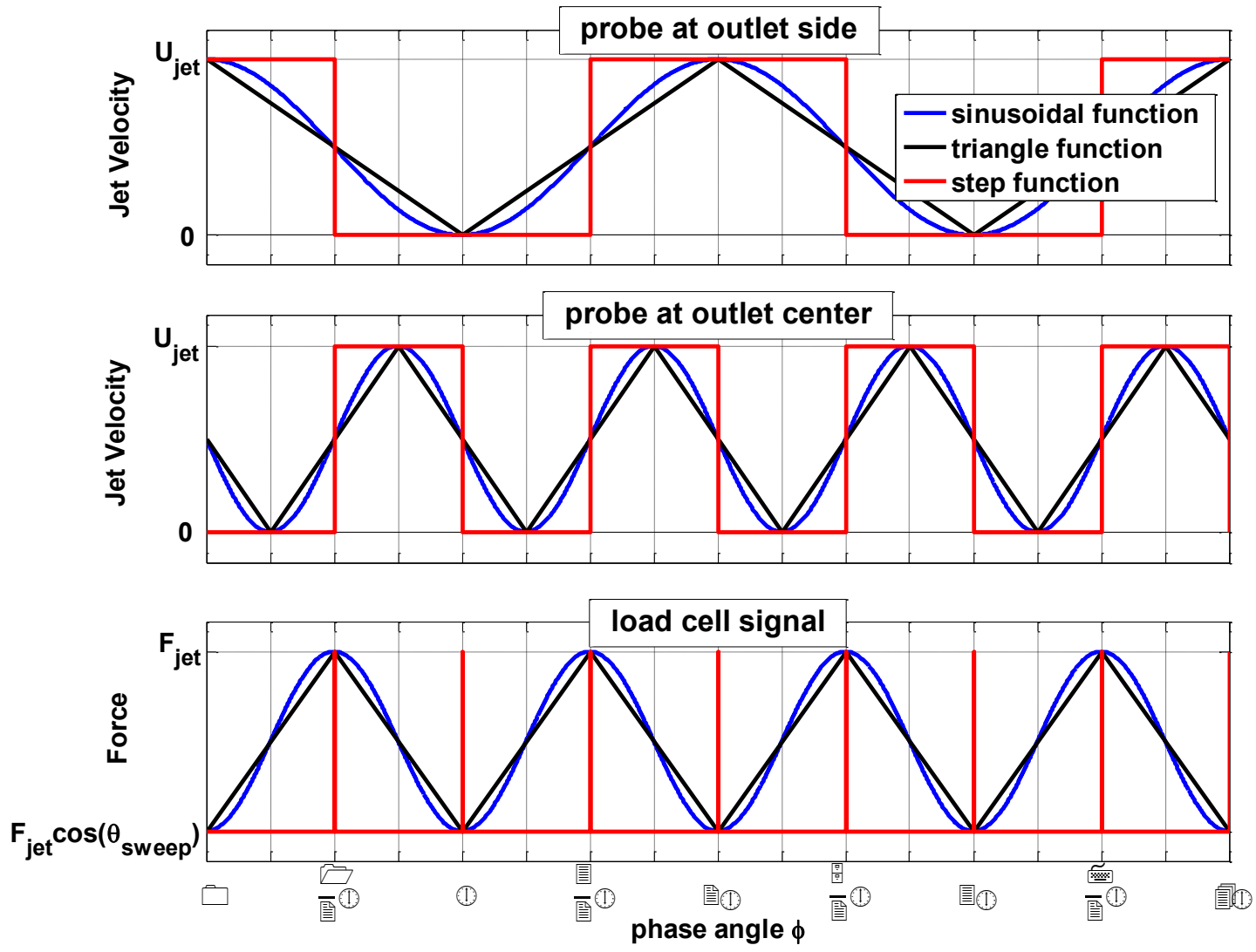

Figure 8 - Approximated hotwire and load cell time signals.

The time signal of a sweeping jet may be described by a sinusoidal behavior (Figure 8). For comparison, Figure 8 also includes a triangle and step function to model 
the sweeping behavior. The angle $\varphi(t)$ represents the phase of the jet throughout one oscillation cycle and ranges from 0 to $2 \pi$. At $\varphi(t)=0$ the jet resides at one end of the sweeping range. The jet's sweeping frequency is based on one period being the complete motion from one side to the other and back, which is correctly depicted in Figure 8 (top) when assuming that the jet's initial position coincides with the probe's position at the outlet's side. However, if the probe were positioned at the outlet's center with the same initial position of the jet the recorded sweeping frequency would be doubled (Figure 8, middle). According to the hotwire signal, the load cell signal for a single actuator would also yield twice the frequency. The maximum force, $F_{j e t}$, is recorded when the jet is aligned with the $x$-direction whereas the minimum force occurs when the jet is located at the side of the sweeping range (i.e., $F_{\text {jet }} \cos \left(\theta_{\text {sweep }}\right)$ ). According to Figure 8 (bottom) the instantaneous force in $x$-direction can be mathematically described by Equation (13) based on the sinusoidal sweeping motion.

$$
F_{\text {measured }}(t)=\underbrace{\frac{F_{\text {jet }}+F_{\text {jet }} \cos \theta_{\text {sweep }}}{2}}_{\text {function offset }}-\underbrace{\frac{F_{\text {jet }}-F_{\text {jet }} \cos \theta_{\text {sweep }}}{2}}_{\text {oscillation amplitude }} \underbrace{\cos (2 \varphi(t))}_{\text {oscillation phase }}
$$

If the measured force is averaged sufficiently long, it is independent of frequency and therefore, it can be obtained by averaging Equation (13) over one period.

$$
\overline{F_{\text {measured }}}=\frac{1}{T} \int_{0}^{T} F_{\text {measured }}(t) d t=\frac{1}{\pi} \int_{0}^{\pi} F_{\text {measured }}(\varphi) d \varphi
$$

Based on Equation (14) the average force ratio is defined as a function of sweeping angle for all wave forms shown in Figure 8. As expected the sinusoidal and triangular function yield the same average jet force. The force ratios are written in a way 
that allows for easy comparison with the extreme case of a step function. Equations (15) and (16) illustrate that the average jet force in $x$-direction is always larger than just the cosine component of the total jet force due the sweeping motion of the jet.

$$
\begin{gathered}
\text { sinusoidal function: } \frac{F_{\text {measured }}}{F_{\text {jet }}}=\cos \theta_{\text {sweep }}+\frac{1}{2}\left(1-\cos \theta_{\text {sweep }}\right) \\
\text { triangle function: } \frac{F_{\text {measured }}}{F_{\text {jet }}}=\cos \theta_{\text {sweep }}+\frac{1}{2}\left(1-\cos \theta_{\text {sweep }}\right) \\
\text { step function: } \frac{F_{\text {measured }}}{F_{\text {jet }}}=\cos \theta_{\text {sweep }}
\end{gathered}
$$

Equations (15) and (17) are plotted in Figure 9 for a wide range of sweeping angles. As the sweeping angle increases the step function approximation diverges significantly from the sinusoidal behavior. Although the outlet geometry of the actuator allows for a sweeping range of $\pm 60^{\circ}$, flow visualization experiments (see Chapter 4.5) indicate a sweeping angle of $\sim 35^{\circ}$ without significant dependence on flowrate ${ }^{[69]}$. For this sweeping angle the mathematical model predicts that $\sim 90 \%$ of the total jet momentum is averaged in $x$-direction which is close to the experimental data (Figure 7). Although the mathematical representation of the sweeping behavior provides valuable approximations of the average jet momentum applied in $x$-direction, it includes simplified assumptions and does not account for certain characteristics of the sweeping jets. For example, it was demonstrated by Gregory et al. ${ }^{[33]}$ that the sinusoidal behavior of the sweeping motion changes with flowrate. However, this simple exercise allows to estimate the axial force from the instantaneous time signal and sweeping angles or to estimate the sweeping characteristics from force measurements. 


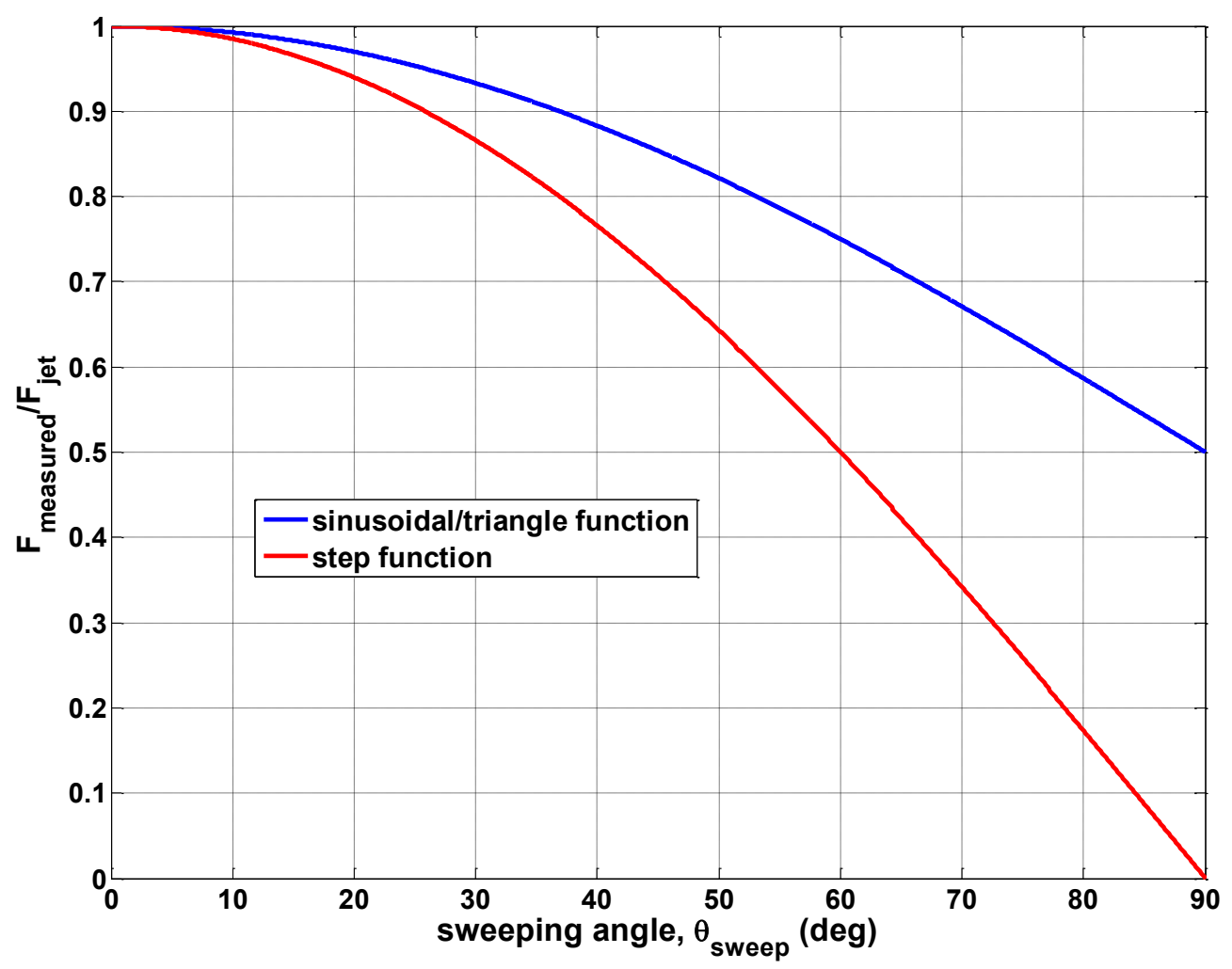

Figure 9 - Calculated force ratio as a function of sweeping angle.

From the aforementioned discussion the question arises whether to consider the total jet momentum or the averaged jet momentum in $x$-direction for the definition of $C_{\mu}$ with respect to separation control. In the presence of a free-stream the emitted jets are expected to be bent in streamwise direction and therefore, more momentum would be applied in $x$-direction. Also, from an efficiency perspective, the total injection of momentum needs to be considered independent of its direction. Because the presented data support the validity of the momentum calculation, the total, calculated momentum is considered to quantify the momentum coefficient. 


\subsection{Effects of Reynolds Number}
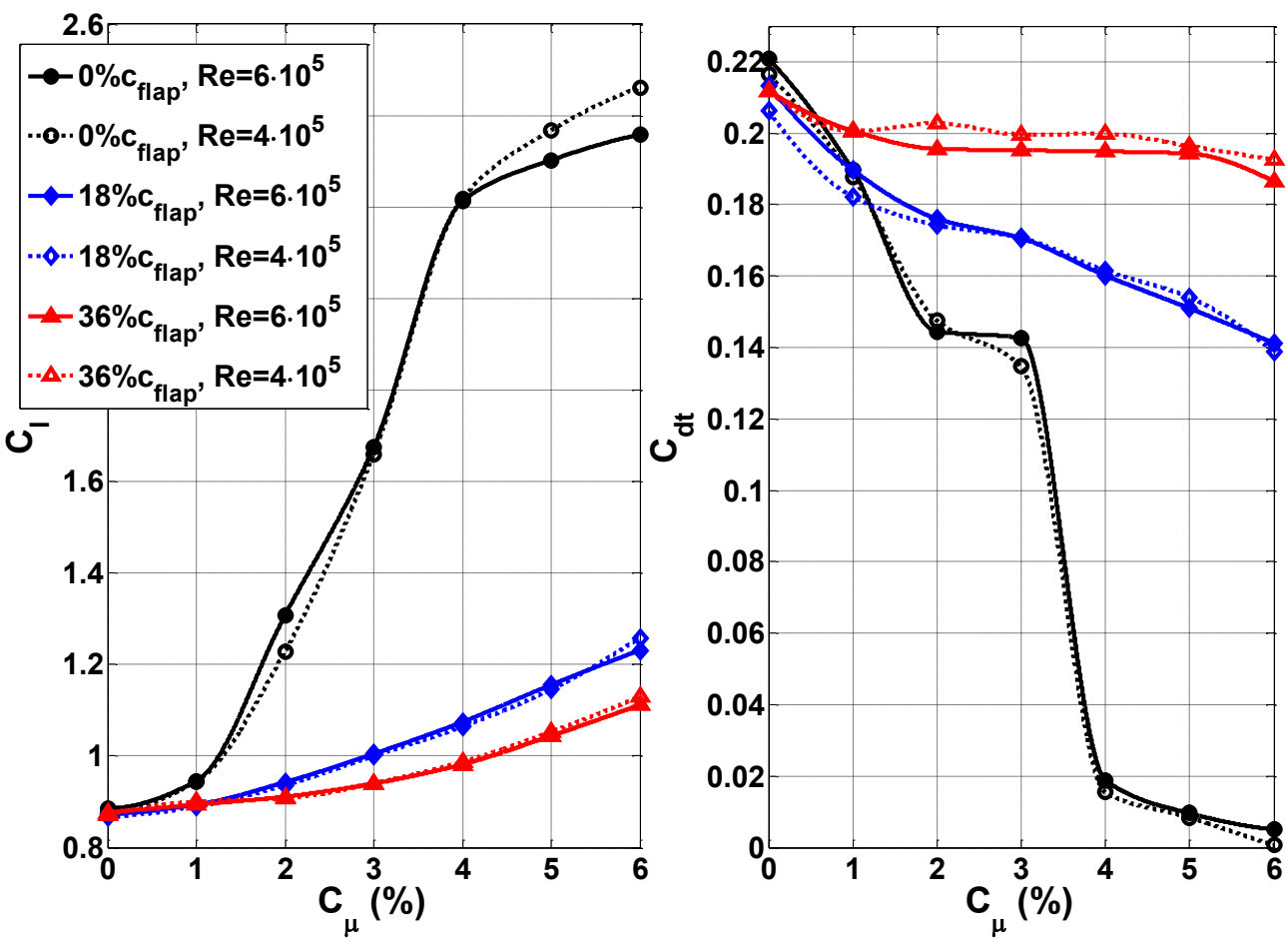

Figure 10 - Flap 60B, effects of $R e, \delta_{T S}=40^{\circ}, \alpha=0^{\circ}, \Delta z=1$ in.

This section addresses the effects of Reynolds number for a fixed actuation configuration (i.e., $\Delta z=$ constant). The baseline flow of every flap configuration was tested at various free-stream velocities to ensure $R e$ independence. The application of sweeping jets was also investigated at different free-stream velocities. Figure 10 illustrates the lift and drag as a function of momentum coefficient for different $R e$ and actuation locations. Only minor deviations between different $R e$ are evident. The total drag, in particular, indicates minor deviations because it is more sensitive to Re effects than lift. Other flap deflections and sizes also reveal only minor $R e$ effects (not shown) when maintaining the same $C_{\mu}$. The agreement between different $R e$ also supports the validity of the $C_{\mu}$ calculations because the jet velocity for $R e=6 \cdot 10^{5}$ is $50 \%$ larger than for $R e=4 \cdot 10^{5}$. Note 
that for a fixed actuator configuration and change in free-stream velocity the consideration of $C_{Q}$ is equivalent to $C_{\mu}$, which means that maintaining the same $C_{\mu}$ for changing $U_{\text {inf }}$ is equivalent to maintaining the same $C_{Q}$. If not stated otherwise, all results presented hereafter are based on $R e=6 \cdot 10^{5}$.

Because the lift obtained for a fixed actuator configuration is directly dependent on the momentum input, it is also dependent on the ratio between jet and free-stream velocity. However, due to the limited free-stream velocities of the wind tunnel, the current experiments are based on velocity ratios of the order of 10 for effective values of $C_{\mu}$. Assuming the jet velocity is bound by sonic speed, the velocity ratio may substantially drop for today's aircraft application. The importance of velocity ratio has been emphasized in studies focused on other active flow control methods. For example, Nagib et al. ${ }^{[7]}$ reported that periodic excitation is strongly dependent on $U_{j e t} / U_{\text {inf }}$ even when considering the same values of $F^{+}$and $C_{\mu}$. Therefore, an increase in the size of sweeping jet actuators to obtain similar values of $C_{\mu}$ or $C_{Q}$ for higher free-stream velocities may not be sufficient to yield similar improvements. A detailed investigation of jet velocity and velocity ratio is needed for proper scaling of sweeping jet actuators.

\subsection{Effects of Hysteresis}

The term hysteresis in aerodynamics refers to a path dependence in changing geometric parameters (e.g., incidence and flap deflection) or actuation parameters (e.g., momentum coefficient). Many actuation methods (e.g., continuous blowing ${ }^{[9]}$, continuous suction $^{[70]}$, and periodic excitation ${ }^{[15]}$ ) inherit a strong hysteresis in controlling flow separation, inferring that these methods require a much smaller level of actuation to 
maintain flow attachment than to reattach separated flow. This has implications for closed-loop control strategies and their capacity to only apply the minimum actuation level necessary. However, it also adds complexity to the control system and requires detailed knowledge of the hysteresis behavior.
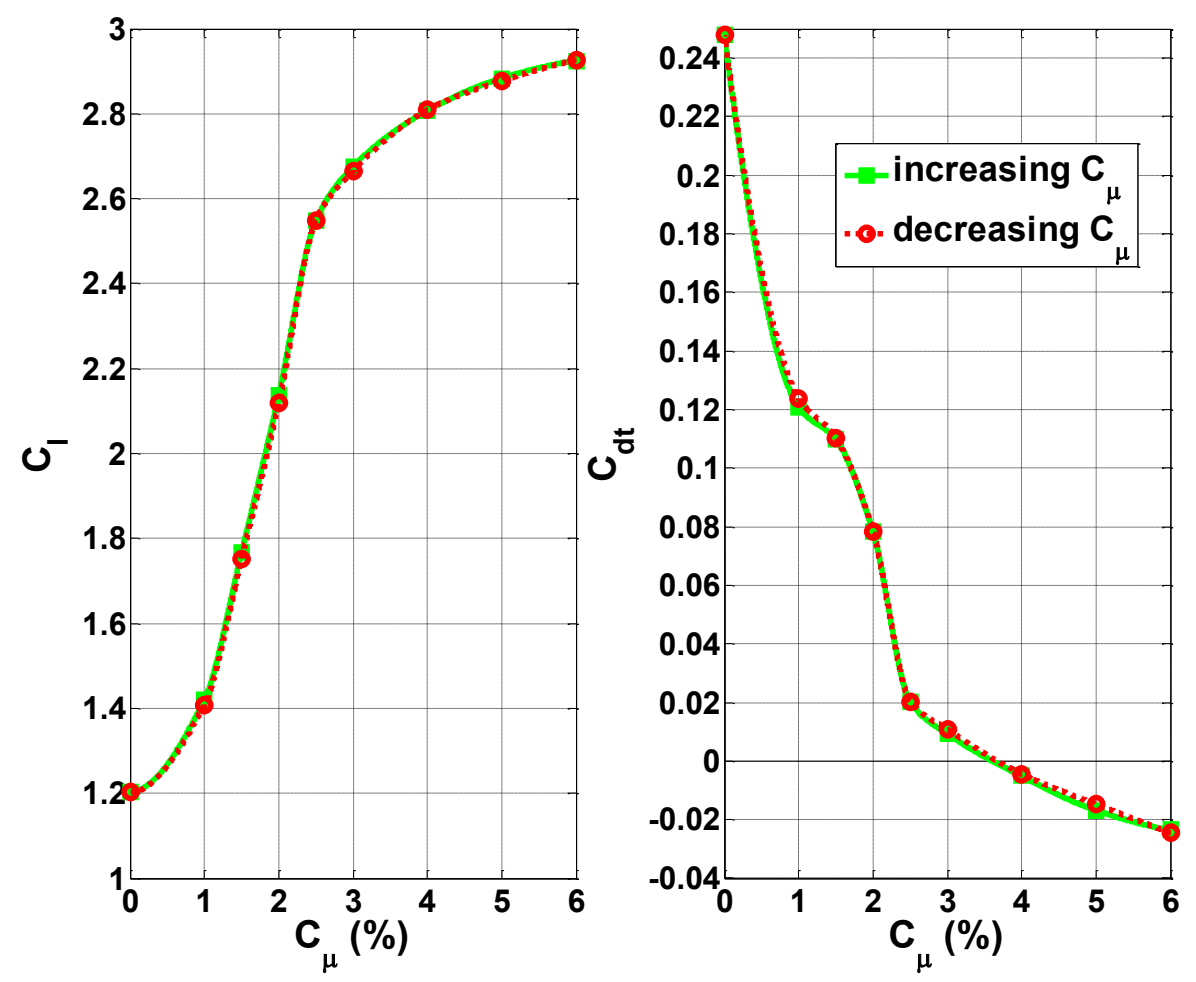

Figure 11 - Flap 40A, hysteresis of changing $C_{\mu}, \delta_{T S}=45^{\circ}, \alpha=0^{\circ}$, AFC @ 7\% $c_{\text {flap }}, \Delta z=1$ in.

For separation control with sweeping jet actuators hysteresis is investigated for changing $C_{\mu}$ at a fixed incidence and for changing incidence at a fixed $C_{\mu}$. Figure 11 illustrates the behavior of lift and drag at $\alpha=0^{\circ}$ if the momentum input is increased and decreased. No hysteresis is evident. The data acquisition was carefully performed to avoid any effects of the experimental methods. Further, the data are repeatable and the same observations are verified for other flap sizes and deflections (e.g., Figure 23). The absence of hysteresis for changing $C_{\mu}$ suggests that the underlying mechanisms of 
separation control with sweeping jets are fundamentally different than continuous blowing and suction, and periodic excitation. These methods target spanwise structures in the flow to control separation whereas sweeping jets target streamwise structures (see Chapter 4.5). It also suggests that sweeping jets are not only effective due to the inserted momentum, but also due to the enhancement or formation of beneficial structures in the flow. If their effectiveness were solely based on the ejected momentum, the fact that separated flow requires more energy to reattach than to stay attached would yield a hysteresis loop. Some evidence for these different underlying mechanisms is presented in Chapter 4.5.

The hysteresis of geometric changes at fixed values of $C_{\mu}$ is illustrated in Figure 12. Solid lines represent an increase in $\alpha$ and dashed lines represent a decrease in $\alpha$. As expected for the baseline flow, the stall angle is $3^{\circ}$ larger when increasing the incidence as opposed to decreasing the incidence. Because actuation is applied from the flap, no significant stall delay is achieved and therefore, the separation and reattachment of the flow from the main element is unaffected by actuation and occurs at the same incidence as for the baseline. Although the flap deflection is held constant, the flow over the flap starts separating before the main element stall as the incidence is increased. Besides small deviations at $C_{\mu}=1 \%$ no hysteresis for flap stall is evident. The current model did not allow for controlled and automated changes of $\delta_{T S}$ while operating the wind tunnel. Therefore, the hysteresis of flap stall could not be investigated in more detail. Future experiments aimed at delaying main element stall may provide additional information about hysteresis effects. 

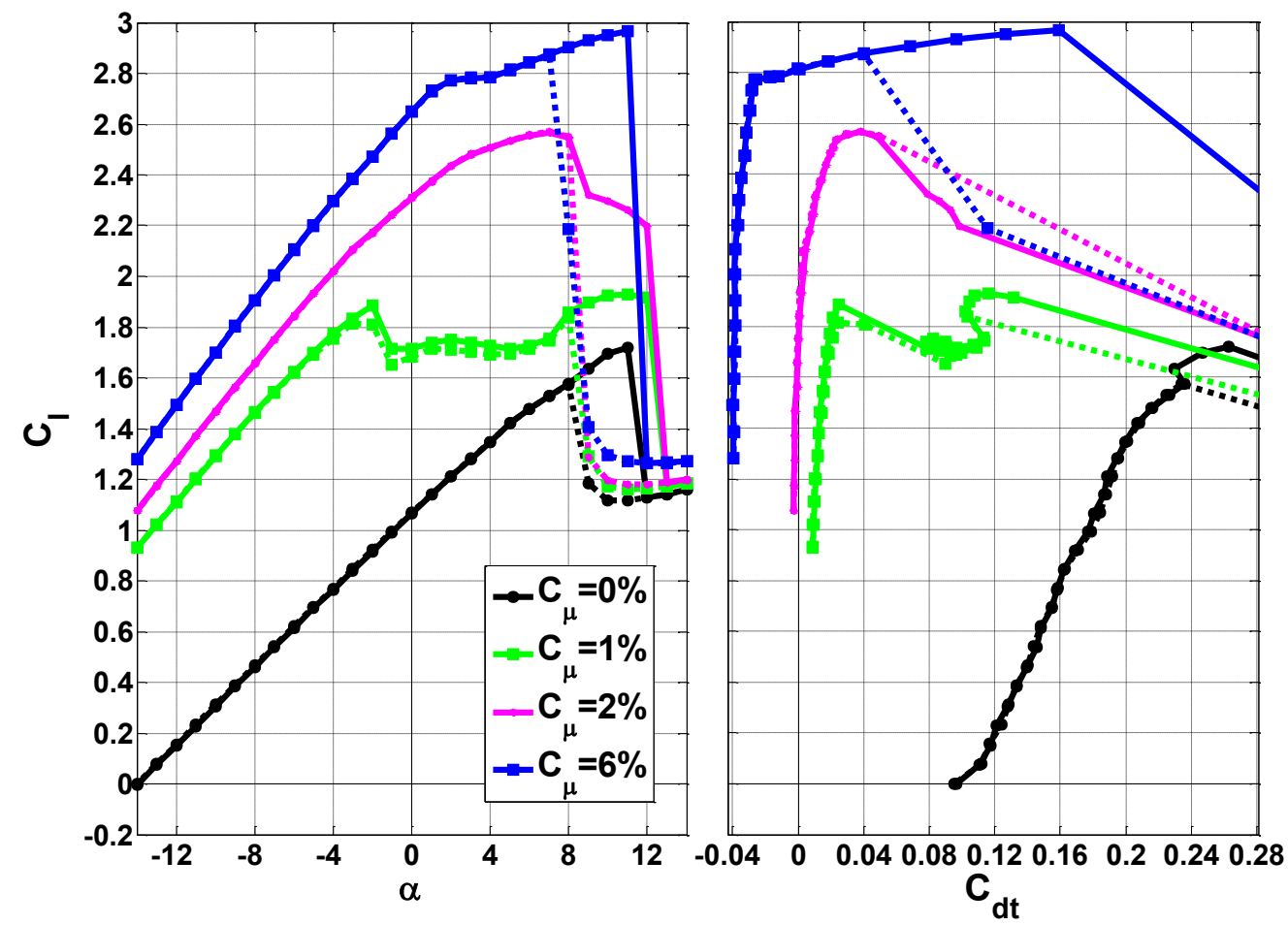

Figure 12 - Flap $40 \mathrm{~A}$, hysteresis of changing $\alpha, \delta_{T S}=40^{\circ}$, AFC @ $7 \% c_{\text {flap }}, \Delta z=1 \mathrm{in}$.

The absence of hysteresis with respect to flap deflection and momentum input simplifies the application of sweeping jet, because the sequence and the direction of changing geometric and actuation parameters is irrelevant to obtain expected flow conditions. Therefore, no complex sequencing and dynamic response as well as closedloop control mechanisms are necessary to achieve optimal performance.

\subsection{Quantification of Actuation Intensity}

The preceding discussion of Reynolds number (Chapter 4.2) establishes that the momentum coefficient governs the model's lift for changing free-stream velocities for a fixed actuator configuration (i.e., $\Delta z=$ constant). However, due to the discrete distribution of momentum with sweeping jets, the question arises whether $C_{\mu}$ is also a governing parameter if the distance between adjacent actuators is changed. 

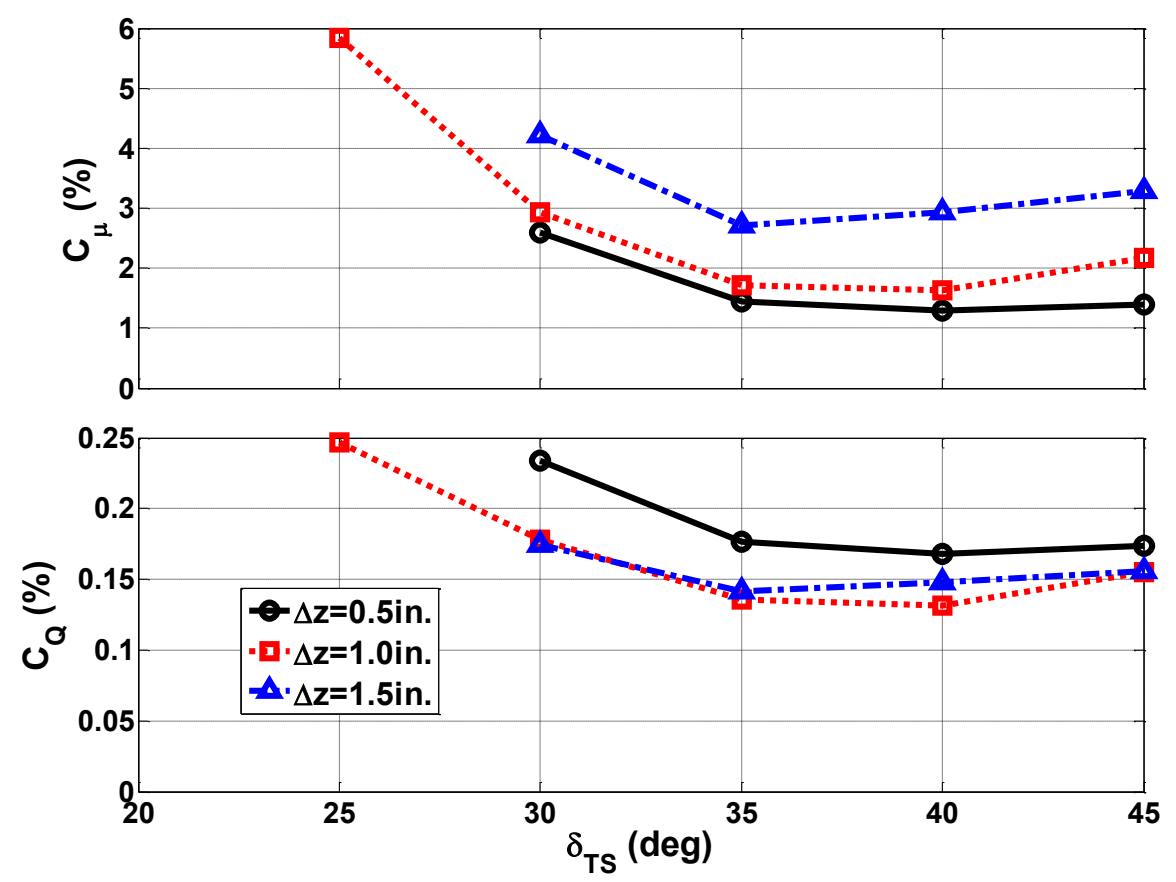

Figure 13 - Flap 40B, required $C_{\mu} \& C_{Q}$ for fixed $C_{l}=1.5, \alpha=0^{\circ}, \mathrm{AFC} @ 4 \% c_{\text {flap }}$.

Figure 13 illustrates the values of $C_{\mu}$ and $C_{Q}$ required to generate $C_{l}=1.5$ as a function of $\delta_{T S}$, inferring that neither $C_{\mu}$ nor $C_{Q}$ are suitable to collapse the data onto a single curve for different values of $\Delta z$. The smallest spacing is most effective when considering the same momentum input; however, the opposite is true when a constant $C_{Q}$ is considered. Note that in some instances the spacing of $\Delta z=1.5 \mathrm{in}$. is less effective than $\Delta z=1$ in. implying that the maximum spacing between actuators is limited. This may be due to limitations in the spanwise extent of the jet penetration at the prescribed velocity ratio. Figure 13 also illustrates how a desired lift can be obtained at various flap deflections with significantly different levels of actuation. For both, $C_{\mu}$ and $C_{Q}$, the minimum is achieved at $\delta_{T S}=40^{\circ}$. For smaller flap deflections a much higher level of actuation is required due to the limited geometric potential to produce lift. For higher flap deflections the actuation intensity increases again because it becomes more difficult to 
overcome separation. The discussion of airfoil configuration, including flap size, shape and deflection, is detailed in Chapter 6.

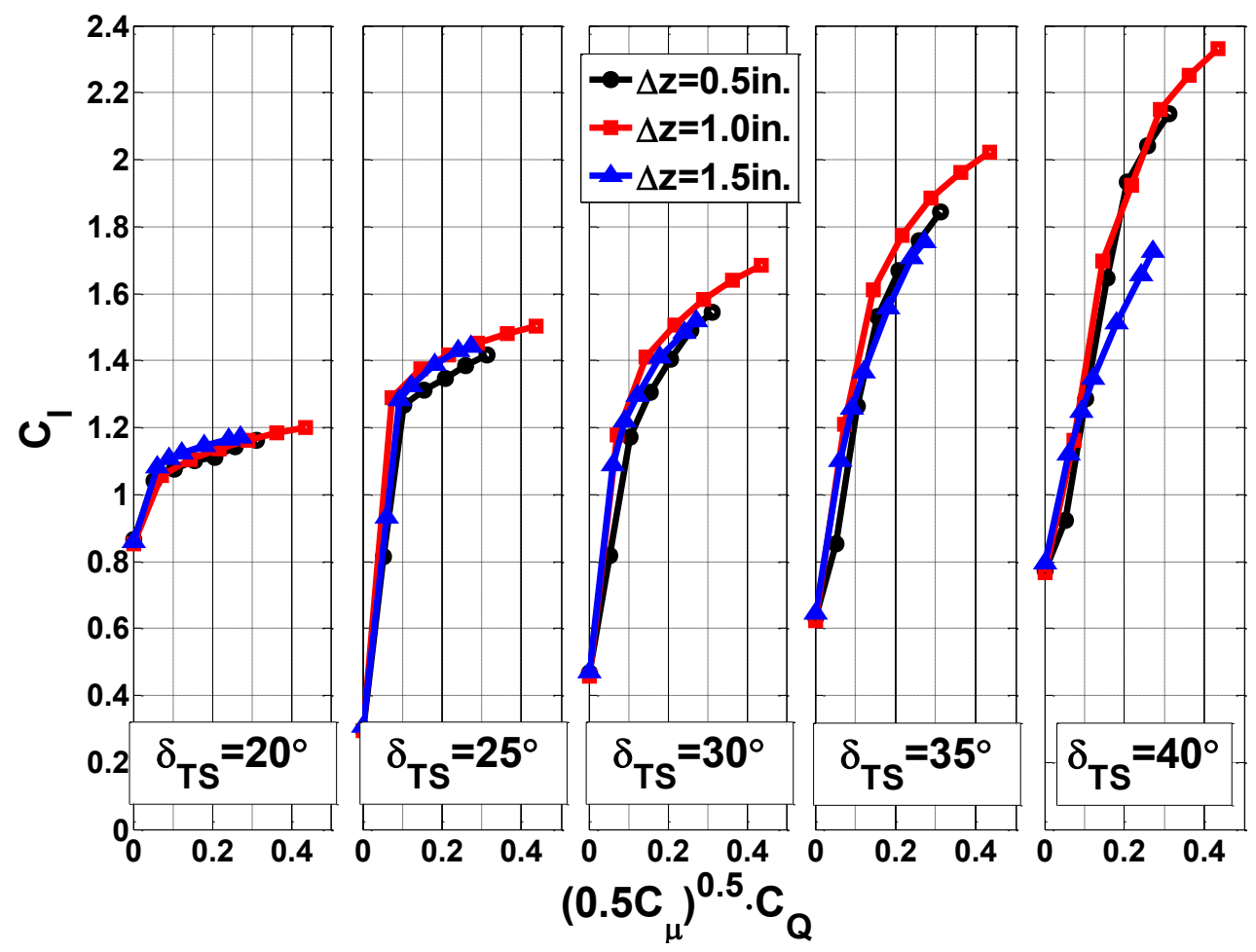

Figure 14 - Flap 40B, actuation intensity for various $\Delta z, \alpha=0^{\circ}, \mathrm{AFC} @ 4 \% c_{f l a p}$.

Because neither $C_{\mu}$ nor $C_{Q}$ appear suitable as a governing parameter by itself, various combinations of the two were investigated (e.g., the combination proposed by Attinello ${ }^{[71]}: 0.5 C_{\mu} C_{Q}$ ). For a wide range of configurations the most success is achieved with the combination proposed in Figure 14. The multiplication of $C_{Q}$ with the square root of $C_{\mu}$ collapses the data well for different nozzle intervals. This coefficient applies for a wide range of flap deflections. However, the data for $\Delta z=1.5 \mathrm{in}$. start deviating at high flap deflections possibly due to the limitations in sweeping extent and exit velocity. Figure 15 and Figure 16 also verify the coefficient $\left(0.5 C_{\mu}\right)^{0.5} \cdot C_{Q}$ for various actuation locations and flap sizes, respectively. Only actuation from the flap shoulder resembles 
larger deviations for $\Delta z=0.5 \mathrm{in}$. due to additional geometric effects specific to this location (Figure 15). The details of these effects are addressed in Chapter 5.1.
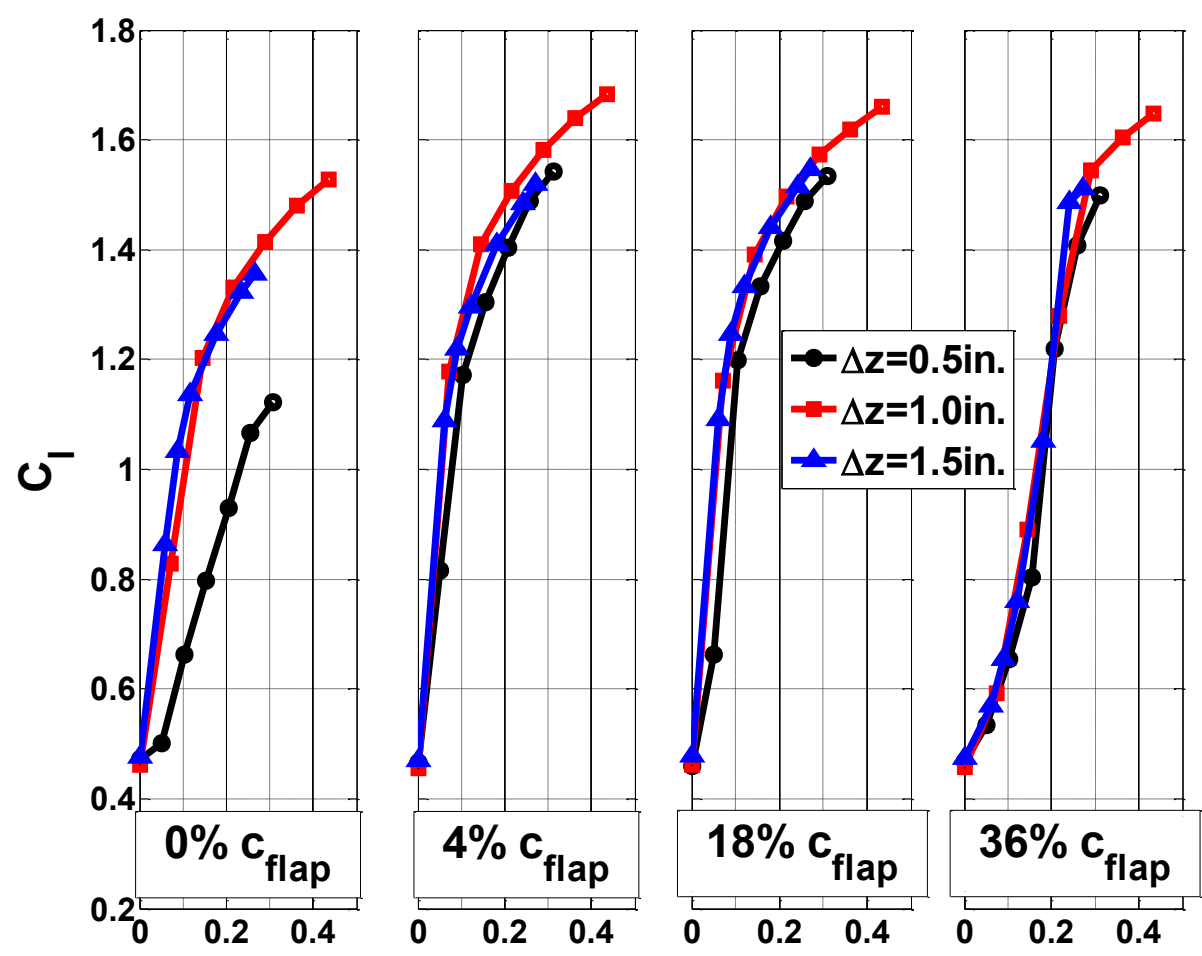

$\left(0.5 C_{\mu}\right)^{0.5} \cdot C_{Q}$

Figure 15 - Flap 40B, actuation intensity for various $\Delta z, \alpha=0^{\circ}, \delta_{T S}=30^{\circ}$.

The proposed coefficient also holds for various flap sizes (Figure 16). However, for the smallest flap size of $20 \% c$ significant deviations are evident for $\Delta z=1.5 \mathrm{in}$. Due to the limitations in spanwise extension of the sweeping jets, the area not covered by the jets at this large spacing is a substantial portion of the small flap. Therefore, the effectiveness in controlling separation may be reduced. Further evidence supporting this argument is presented in Chapter 4.6.2. Although the applicability of $\left(0.5 C_{\mu}\right)^{0.5} \cdot C_{Q}$ is validated for various flap deflections, flap sizes, and actuation locations, it remains to be verified for larger size models and actuators. The total drag data cannot be collapsed by this 
coefficient because the reduction in drag is directly related to the inserted momentum ${ }^{[66,67]}$. Once the flow is fully attached, the additional momentum input is almost fully recovered in thrust which is addressed in several subsequent sections.
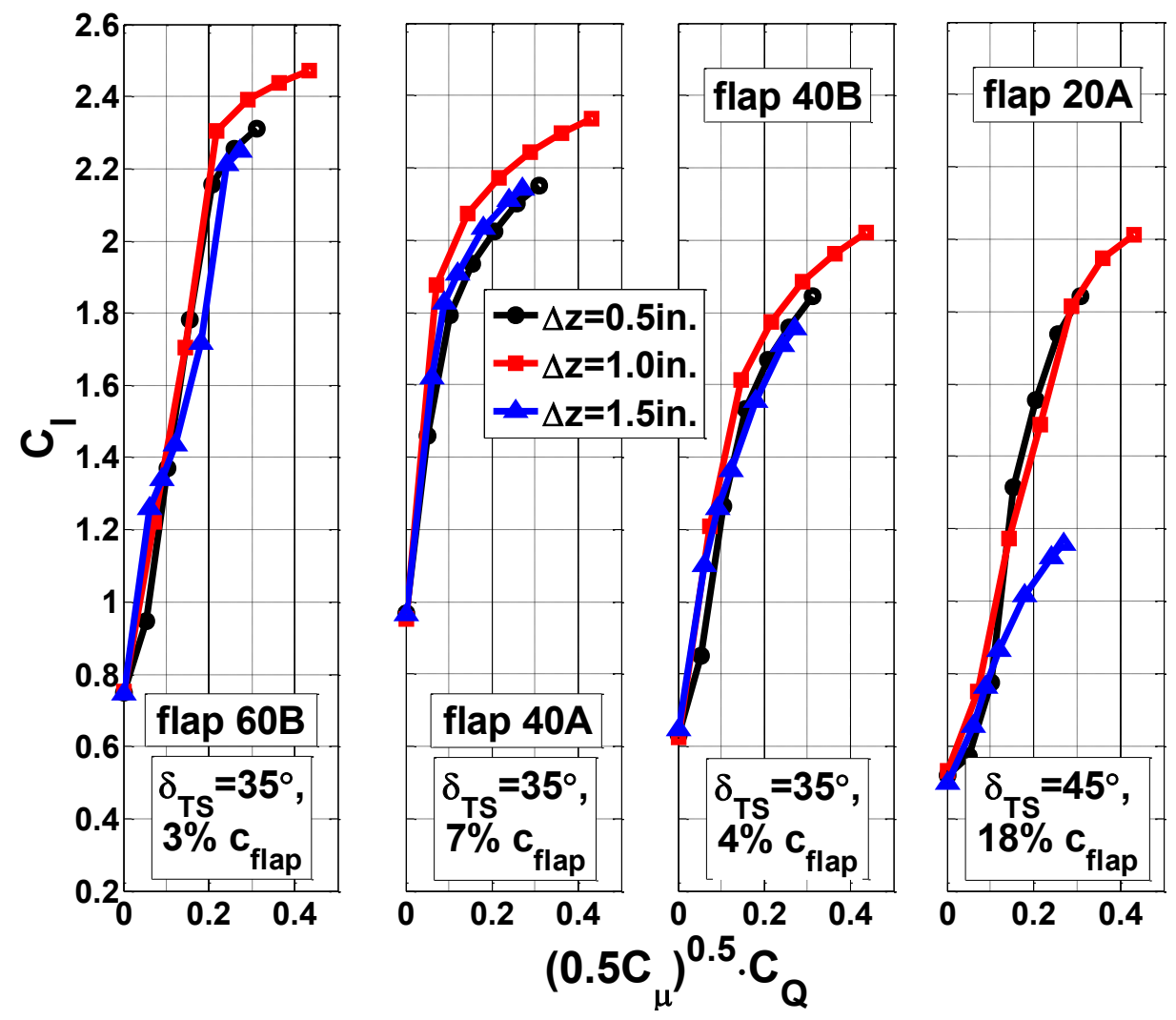

Figure 16 - Actuation intensity for various $\Delta z \&$ flap sizes, $\alpha=0^{\circ}$.

The proposed coefficient provides useful indications to aid the scaling of sweeping jet actuators. The combination of $C_{\mu}$ and $C_{Q}$ may also be expressed only in terms of $C_{Q}$ (Equation (18)) or $C_{\mu}$ (Equation (19)). Equation (18) indicates that the actuation intensity may be increased more effectively by increasing $C_{Q}$ and not by increasing $\Delta z$. For example, if $C_{Q}$ is doubled the coefficient $\left(0.5 C_{\mu}\right)^{0.5} \cdot C_{Q}$ is quadrupled and if $\Delta z$ is doubled the coefficient only increases by $41 \%$. Increasing $C_{Q}$ instead of $\Delta z$ also reduces the total drag which is directly related to the momentum input applied. 
Doubling $C_{Q}$ increases $C_{\mu}$ by a factor of four while doubling $\Delta z$ only increases $C_{\mu}$ by a factor of two. Note that the actuation intensity is still dominated by the velocity ratio squared and therefore by the momentum input. Only the weight of the area ratio is increased by its square root.

$$
\begin{gathered}
\left(0.5 C_{\mu}\right)^{0.5} C_{Q}=\left(\frac{C_{Q}^{2}}{n A_{\text {nozzle }} / A_{\text {ref }}}\right)^{0.5} C_{Q}=\left(\frac{A_{\text {ref }}}{n A_{\text {nozzle }}}\right)^{0.5} C_{Q}^{2}=\left(\frac{c \Delta z}{A_{\text {nozzle }}}\right)^{0.5} C_{Q}^{2} \\
\left(0.5 C_{\mu}\right)^{0.5} C_{Q}=\left(0.5 C_{\mu} \cdot \frac{n A_{\text {nozzle }}}{A_{\text {ref }}} C_{\mu}\right)^{0.5}=\left(0.5 \frac{n A_{\text {nozzle }}}{A_{\text {ref }}}\right)^{0.5} C_{\mu}=\left(0.5 \frac{A_{\text {nozzle }}}{c \Delta z}\right)^{0.5} C_{\mu}
\end{gathered}
$$

The coefficient $\left(0.5 C_{\mu}\right)^{0.5} \cdot C_{Q}$ also has implications for the dependence on the sweeping frequency. Assuming a constant value of $\left(0.5 C_{\mu}\right)^{0.5} \cdot C_{Q}$, the total flowrate into the system is only reduced by $16 \%$ when $\Delta z$ is doubled. However, because the number of actuators is halved, the flowrate per actuator is increased by $68 \%$. Recalling the almost linear relationship between sweeping frequency and flowrate per actuator the sweeping frequency is significantly higher for the larger actuator spacing. This indicates that the effectiveness of the sweeping jets does not significantly depend on the sweeping frequency when being of the order of $\mathrm{kHz}$. This argument is supported by DeSalvo et al. ${ }^{[60]}$ who base their discussion on $F^{+}$being an order of magnitude higher than the commonly used $F^{+}=O(1)$. As long as the sweeping frequency is an order of magnitude higher than any dominant frequencies in the natural flow, the exact value of $f_{\text {sweep }}$ is not important. However, a detailed understanding of the transient processes in controlling flow separation with sweeping jets is necessary to further unpack this discussion. 


\subsection{Surface Flow Visualization}

In order to fully address the question of how actuation with sweeping jets should be applied, a detailed understanding of the underlying mechanisms controlling separation is necessary. Although no time-resolved data of the complete flow field is currently available, the quantitative discussion of global governing parameters is complemented by surface flow visualization results which provide suggestions for these mechanisms. Some selective photographs are shown for the flap configuration 40B with actuation from the flap shoulder at $\delta_{T S}=30^{\circ}$ and $\Delta z=1$ in. This flap deflection appears to be most suitable and of most interest due to the presence of strongly separated flow, large effectiveness of actuation, and reasonable gravity effects. The angle of attack is fixed at $0^{\circ}$. Since surface flow visualization only provides time-averaged information of the flow's traces on the surface, its interpretation involves some ambiguity. Structures away from the surface may only be evident through their traces on the surface. All photographs only show the model's flap or enlargements thereof. The free-stream is always directed from the top to the bottom in the pictures. Each photograph contains a horizontal scale which is also valid for the vertical direction.

Figure 17 illustrates the entire length of the flap for the baseline case. Note the indication of the main element's and flap's trailing edge, as these are omitted in subsequent figures. The flow is clearly separated as suggested by the pressure data (not shown). However, the separation location (marked, thick white line) appears to be distinctively downstream of the discontinuity at the flap shoulder and therefore downstream of the actuation location. The upstream thin white line is caused by an 
accumulation of fluid running toward the inside of the flap shoulder cavity. This line is not present when applying less fluid or applying it more carefully. A balance between gravity, forcing the fluid downstream, and re-circulation, forcing the fluid upstream, yields the somewhat random structures close to the flap's trailing edge.

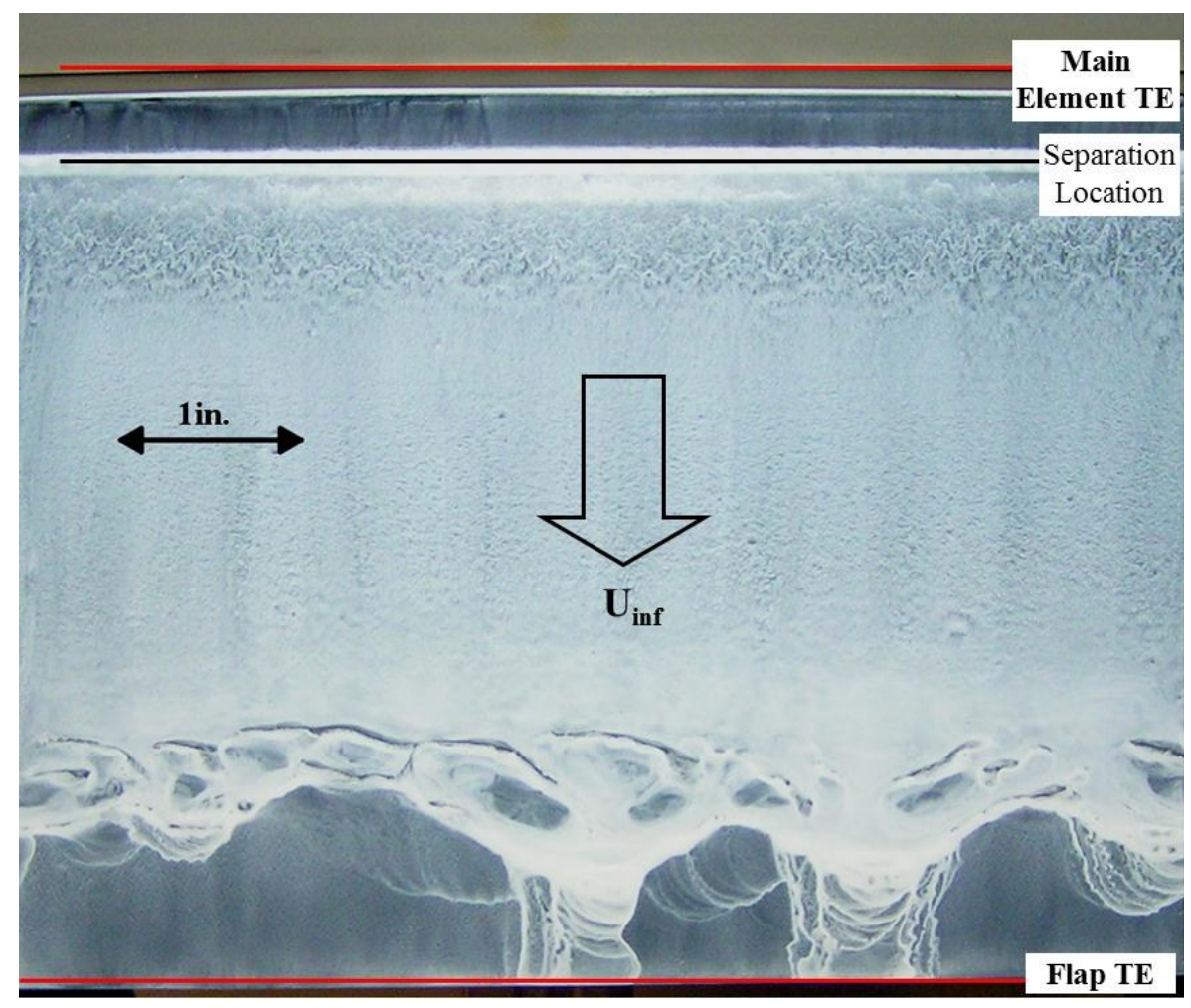

Figure 17 - Surface flow visualization, $\alpha=0^{\circ}, \delta_{T S}=30^{\circ}$, baseline.

Applying actuation with $C_{\mu}=3 \%$ yields a very different picture than the baseline by attaching the flow over the flap (Figure 18, left). The vast accumulation of fluid due to recirculation is prevented. The interesting part of Figure 18 (left) is revealed when enlarging the region just downstream of the actuation location (Figure 18, right) where a continuous stream of fluid appears to be wrapped up in some form of necklace vortex in between adjacent actuators. The necklace vortex is caused by the formation of a pair of 
counter-rotating vortices normal to the surface. The vortices likely originate from the high velocity jets and manifest themselves at the boundary of the jets' sweeping range. The initially normal vorticity is being bent in streamwise direction by the free-stream evident by surface traces reaching all the way to the flap's trailing edge (Figure 19). A wake downstream of the vortex pair is also apparent.

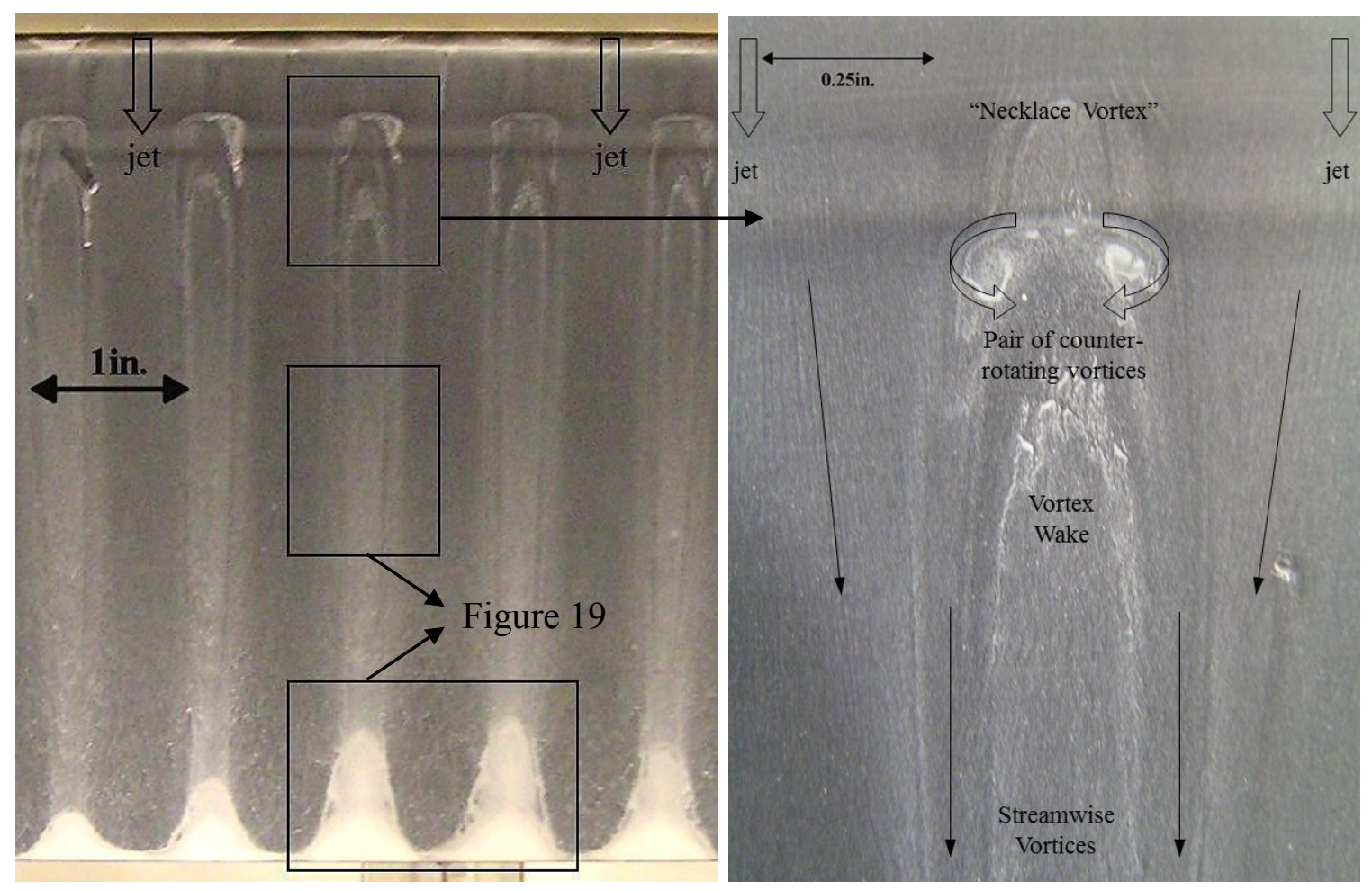

Figure 18 - Surface flow visualization, $\alpha=0^{\circ}, \delta_{T S}=30^{\circ}, \mathrm{AFC} @ 0 \% c_{\text {flap }}, C_{\mu}=3 \% \Delta z=1$ in., entire flap (left), close-up view at actuator exit (right).

The location where the pair of vortices forms agrees well with the expected interaction zone of adjacent jets considering a sweeping range of $\pm 40^{\circ}$. The location of the vortex pair does not change noticeably by increasing $C_{\mu}$ (Figure 20, right), and neither does the sweeping angle. This suggests that the vortices form due to the interaction of neighboring jets. As one jet is emitted at an angle to the free-stream direction it will eventually propagate into the impact zone of its neighboring jet. The according adverse 
pressure gradient pushes the fluid away from the jet's initial direction and therefore, rolls up into a vortex. The same scenario affects the neighboring jet forming a vortex with opposite sense of rotation. The normal vorticity forming through this interaction is then bent in streamwise direction developing a pair of counter-rotating streamwise vortices, which moves high velocity fluid from the outer flow region to the surface and low velocity fluid away from the surface. Although the initial location of the pair of vortices does not seem to change, the initial distance between the two vortices appears to become smaller with increasing $C_{\mu}$ due to the jet's increasing velocity (cf. Figure 18, right and Figure 20, right).
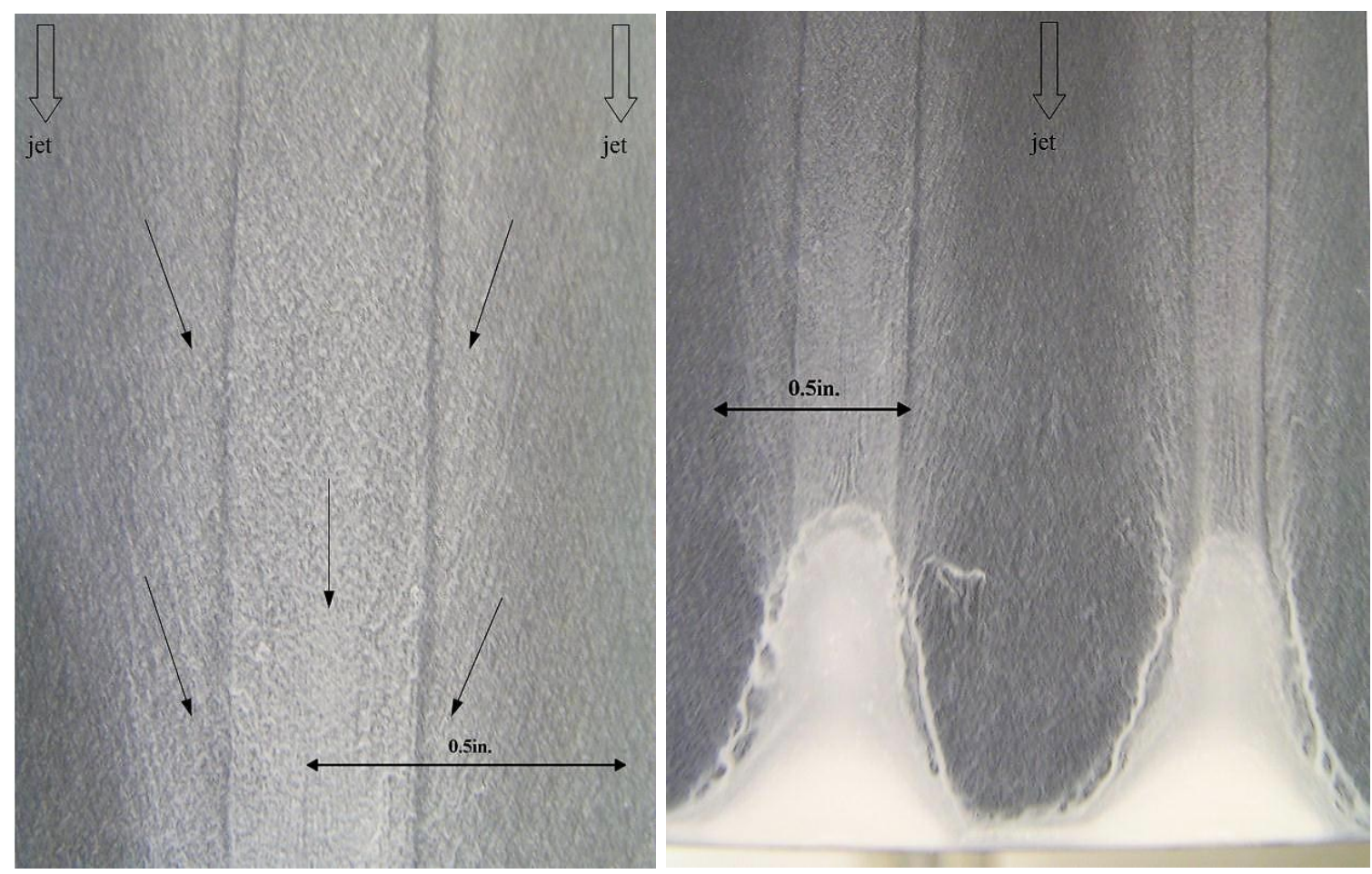

Figure 19 - Surface flow visualization, $\alpha=0^{\circ}, \delta_{T S}=30^{\circ}, \mathrm{AFC} @ 0 \% c_{f l a p}, C_{\mu}=3 \%, \Delta z=1$ in., streamwise streaks down to flap TE.

As the jets sweep toward the symmetry line of the actuator, the downstream distance before interacting with the vortex pair increases, thereby continuously feeding 
into the streamwise vorticity over a long downstream distance (Figure 19, left). This pattern is repetitive along the span. Figure 19 visualizes the traces of the suggested streamwise vortices reaching all the way to the flap's trailing edge. Due to the sense of rotation more fluid is pushed toward the middle of the vortex pair thereby providing a brighter region in between them. The direction of the time-averaged surface traces indicate that the jet flow is moving toward the vortices at an angle as expected from the jets' sweeping motion. The average direction of the flow in between the vortices appears to be in streamwise direction. Potential flow theory for these pairs of counter-rotating vortices suggests that, depending on their strength, and initial distance from one another and from the wall, they will move closer together and away from the wall which is evident in Figure 18. This is even more apparent when increasing $C_{\mu}$ to $6 \%$ (Figure 20). The separation from the surface and eventually the flap's trailing edge is resembled by the wavy accumulation of fluid at the trailing edge. These regions become smaller with increasing $C_{\mu}$ (cf. Figure 18, left and Figure 20, left), suggesting that the pair of streamwise vortices is maintained further downstream and at a higher strength.

Although the location where the vortex pair originates does not change with $C_{\mu}$, the surrounding flow patterns appear altered (cf. Figure 18, right and Figure 20, right). For a higher $C_{\mu}$ a zone of strong flow entrainment is noticeable upstream of the vortex pair (Figure 20, right) and the details of the vortex wake are not as pronounced. This may be explained, in part, by the uncertainty of the flow visualization technique. Because the high velocity jets quickly push the fluid away before it can dry, small details may not be visible. 

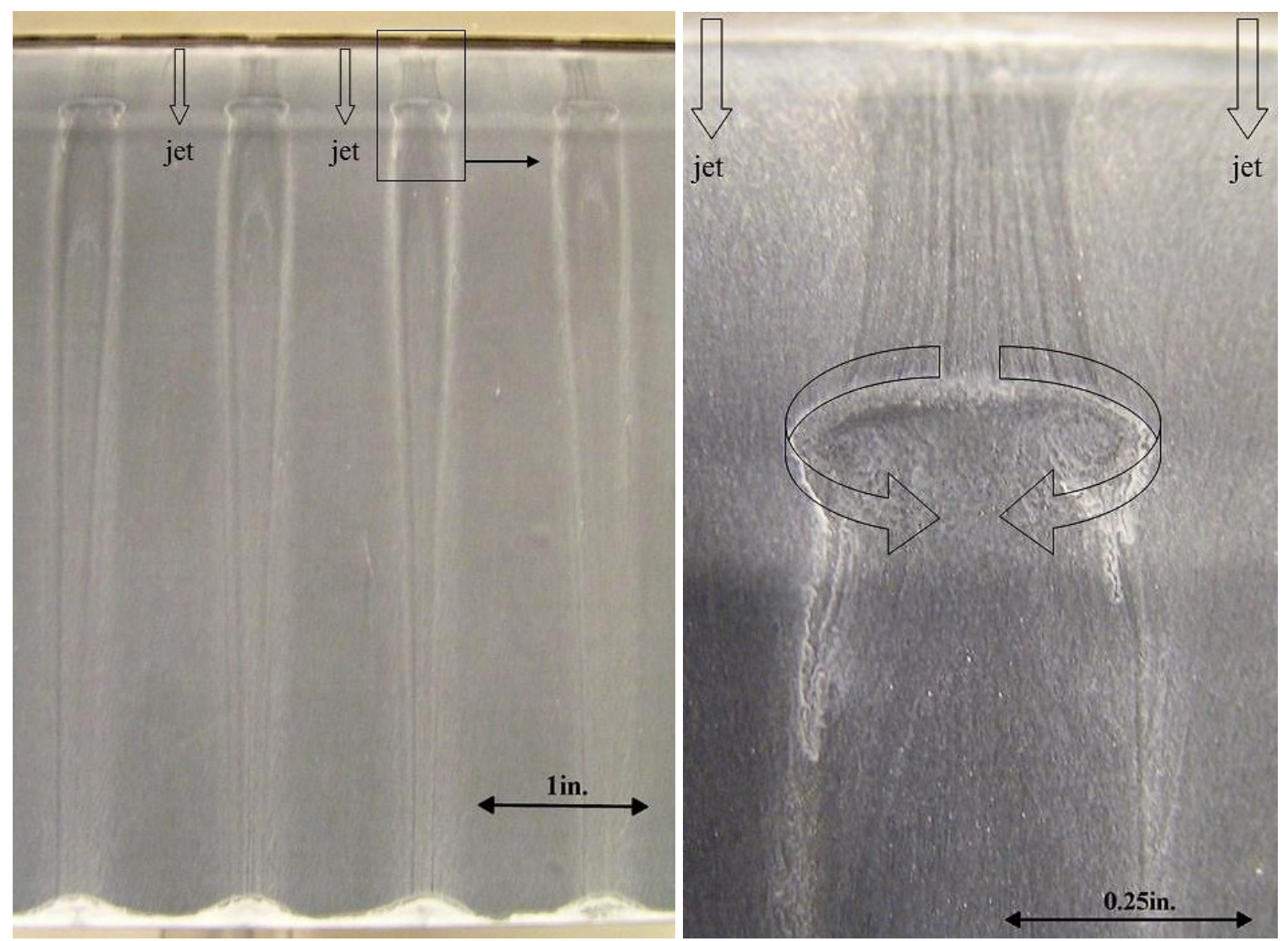

Figure 20 - Surface flow visualization, $\alpha=0^{\circ}, \delta_{T S}=30^{\circ}, \mathrm{AFC} @ 0 \% c_{f l a p}, C_{\mu}=6 \%, \Delta z=1$ in., entire flap (left), close-up view at actuator exit (right).

The discussion presented in this section is supported by experiments with microjets. Although these jets do not inherit any sweeping motion, their mechanisms may be similar to sweeping jets. $\operatorname{Kumar}^{[12]}$ presented evidence for streamwise vortices through surface flow visualization and cross-sectional PIV measurements. He also reasons that the streamwise vortices enhance the mixing between the boundary layer and the outer flow regime and thereby control flow separation. Additional data and flow visualization pictures are discussed in the following chapter with respect to a comparison between sweeping and non-sweeping jets. 


\subsection{Sweeping vs. Non-Sweeping Jets}

The study of sweeping jets begs the question of why sweeping jets ought to be used for separation control instead of just steady, non-oscillating jets. Other researchers ${ }^{[11-13,59,60]}$ have already demonstrated promising results with steady jets. For example, DeSalvo et al. ${ }^{[59,60]}$ claim that steady jets from diverging nozzles perform better than the particular sweeping jet actuators they were using. Therefore, this chapter presents a quantitative and qualitative comparison between sweeping and non-sweeping jets. This discussion also provides an example for a possible failure mode of sweeping jet actuators due to clogged feedback channels. The flap configuration 20A with actuation from $18 \% c_{f l a p}$ was chosen for this comparison because multiple models of this flap were available and actuation from this location is most effective with sweeping jets. In order to inhibit the jets' oscillations, an access to each feedback channel was drilled through which the channels were carefully filled with epoxy. It was ensured that no epoxy entered the main chamber of the actuators to allow for a proper comparison. The access holes were sealed and sanded to provide a smooth surface finish.

\subsubsection{Quantitative Comparison}

The first step of comparing sweeping with non-sweeping jets is based on a quantitative discussion. If separation control with non-sweeping jets is comparable to sweeping jets, the properties discussed in the previous chapters may also apply. The coefficient $\left(0.5 C_{\mu}\right)^{0.5} \cdot C_{Q}$ governing the actuation intensity with sweeping jets is evaluated in Figure 21. The agreement between different actuator spacings is only satisfactory for small levels of actuation. The more actuation is applied, the more the curves diverge 
identifying actuation with $\Delta z=0.5 \mathrm{in}$. as the most effective with the highest lift improvement.
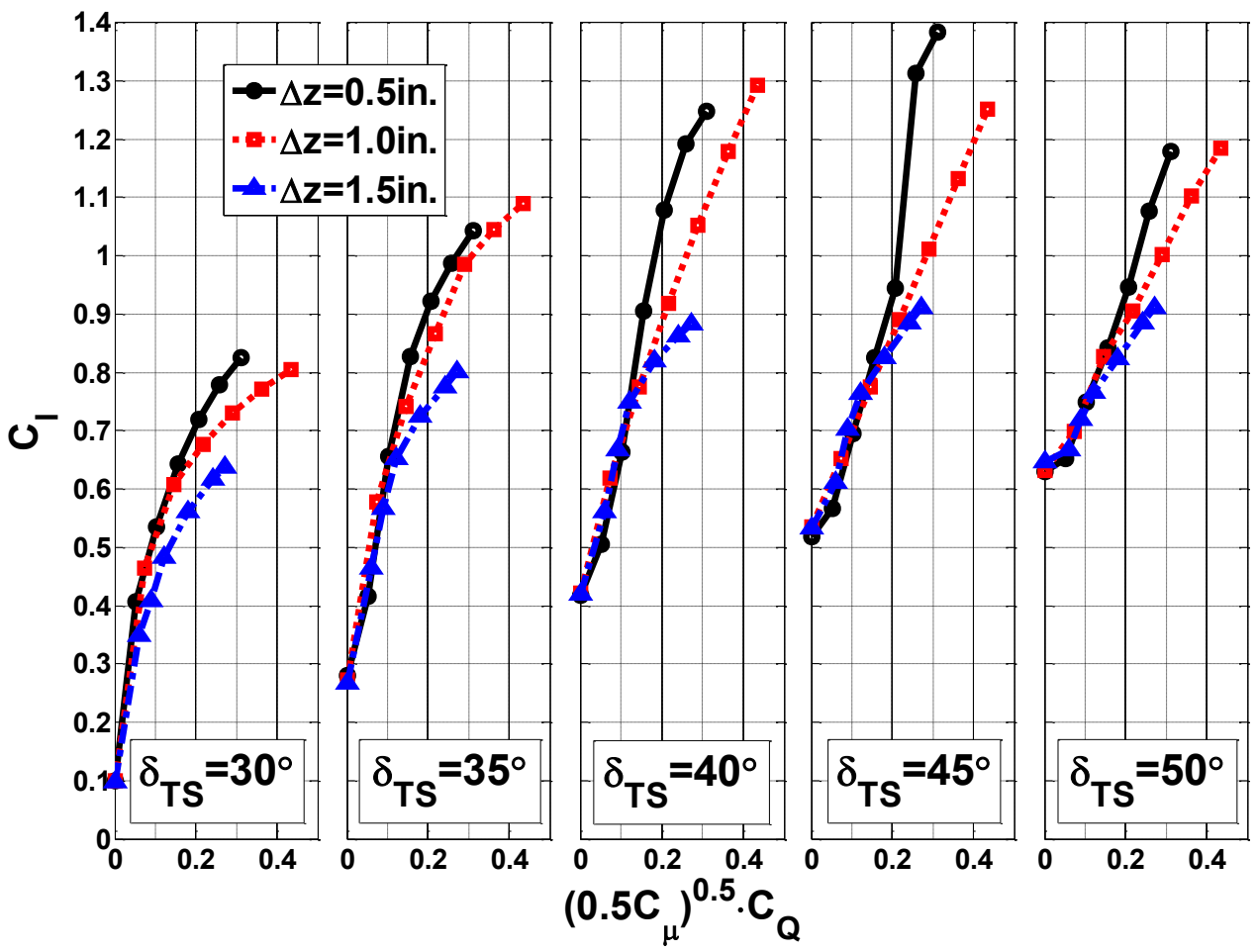

Figure 21 - Flap 20A, lift vs. actuation intensity, $\alpha=0^{\circ}$, non-sweeping jets, AFC @ 18\% $c_{\text {flap }}$.

Since the coefficient $\left(0.5 C_{\mu}\right)^{0.5} \cdot C_{Q}$ does not seem to be applicable for nonsweeping jets other coefficients were tested. The momentum coefficient results in even larger differences between various $\Delta z$ (not shown). The best agreement, especially for higher levels of actuation, is provided by the flowrate coefficient, $C_{Q}$ (Figure 22). This implies that for non-sweeping jets to be effective an increase in $\Delta z$ has to be compensated by a proportional increase in flowrate per actuator and therefore jet velocity. If fewer jets control separation, the jets need to be stronger accordingly. The trend that the effectiveness of steady jets is reduced with increasing distance between them indicates the main difference with sweeping jets. The impact area of steady jets is strongly 
dependent on the jets' strength and spacing whereas the impact area of sweeping jets is enhanced by the sweeping motion, which yields more effective and efficient separation control. Additional evidence for a higher efficiency is presented shortly.

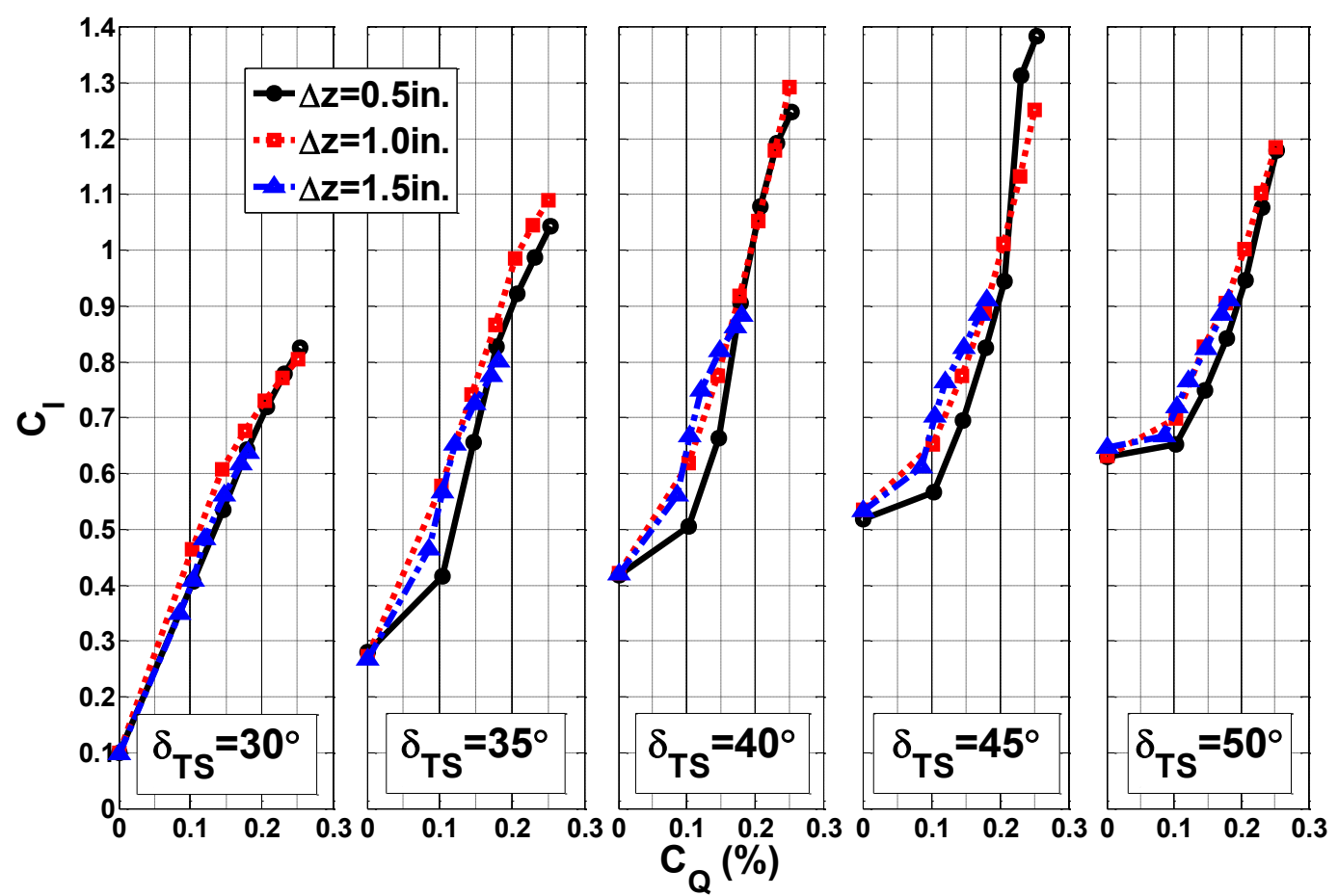

Figure 22 - Flap 20A, lift vs. flowrate coefficient, $\alpha=0^{\circ}$, non-sweeping jets, AFC @ $18 \% c_{\text {flap }}$.

Another useful comparison between sweeping and non-sweeping jets is illustrated in Figure 23 which addresses hysteresis effects. As shown in Chapter 4.3, no $C_{\mu}$ hysteresis in lift and drag is evident for sweeping jets. Minor hysteresis effects are apparent for non-sweeping jets especially in the drag data. However, unlike traditional flow control methods, the hysteresis behavior indicates a better performance when increasing $C_{\mu}$ instead of decreasing $C_{\mu}$. The absence of strong hysteresis behavior for both sweeping and non-sweeping jets indicates that both methods inherit similar underlying mechanisms as suggested by $\operatorname{Kumar}^{[12]}$. The qualitative discussion of these 
mechanisms is addressed in more detail in the subsequent chapter. Figure 23 also shows that actuation with sweeping jets requires a smaller momentum input to attach the flow and yields a much larger lift. This implies that even though both methods are capable of attaching the flow over the flap, sweeping jets achieve higher lift improvements due to an increased upstream effect. Although the lift values differ, the total drag values are comparable at high momentum input. Note that the inserted momentum with sweeping jets is entirely recovered in thrust once the flow over the flap is fully attached. This further supports the validity of the $C_{\mu}$ calculations (Chapter 4.1).
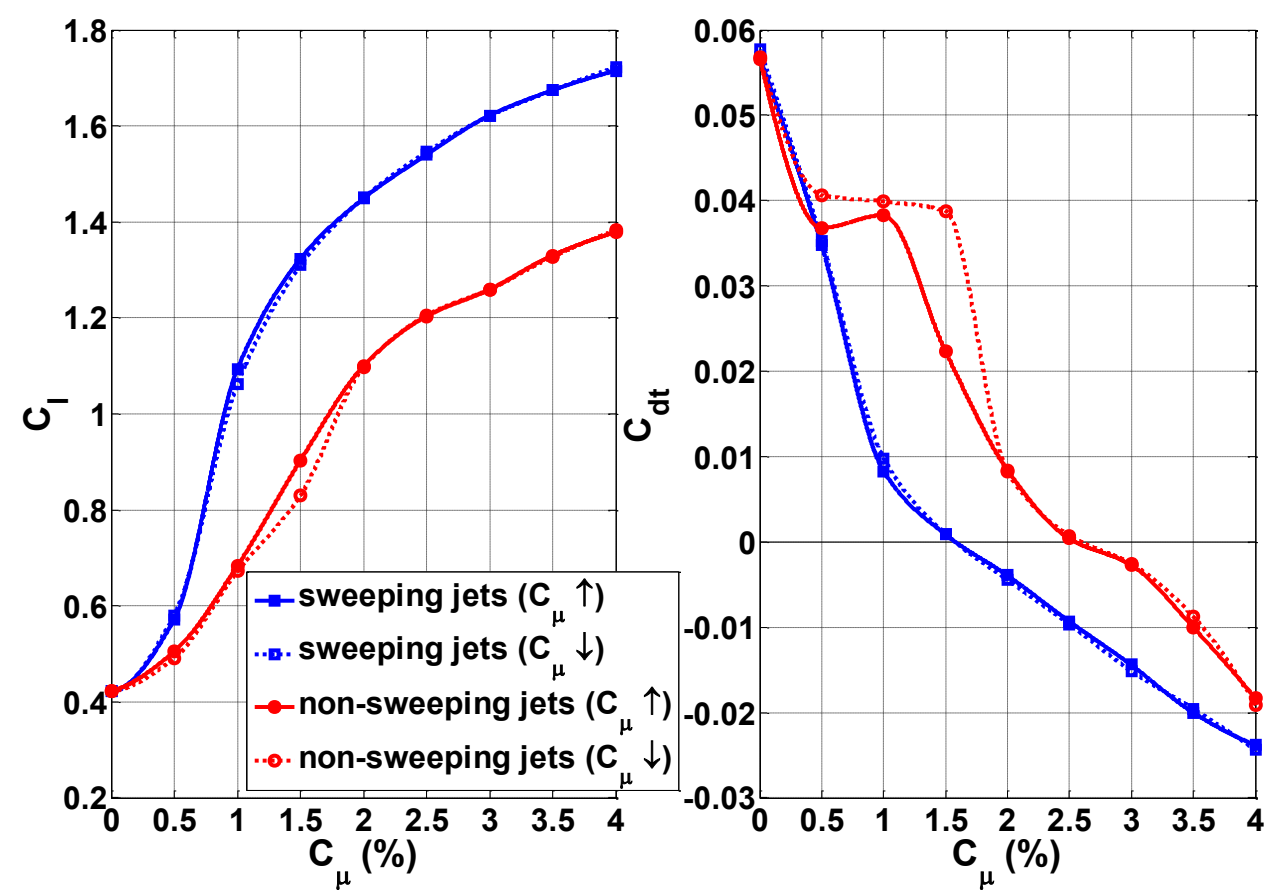

Figure 23 - Flap 20A, hysteresis of changing $C_{\mu}, \delta_{T S}=40^{\circ}, \alpha=0^{\circ}, \mathrm{AFC} @ \sim 20 \% c_{\text {flap }}, \Delta z=1 \mathrm{in}$.,

Although both methods yield improvements in lift and drag, the main question is which method provides these improvements most efficiently. The efficiency is evaluated based on the smallest corrected drag at a prescribed lift value. The first assessment of efficiency is summarized in Figure 24 which is based on the momentum efficiency $\eta_{C_{\mu}}$ as 
defined by Equation (9). Here the momentum input is added to the drag. For a desired lift value the combination of $C_{\mu}$ and $\delta_{T S}$, which yields the smallest corrected drag (i.e., $C_{d t}+C_{\mu}$ ) and therefore the highest value of corrected lift-to-drag ratio (i.e., $\eta_{C_{\mu}}$ ), is found for $\Delta z=0.5 \mathrm{in}$. and $\alpha=0^{\circ}$. Actuation with sweeping jets is quickly identified as the more efficient method. Not only do sweeping jets yield a smaller corrected drag and require a smaller $C_{\mu}$, they also provide lift values almost 50\% larger than with non-sweeping jets. However, compared to the baseline, even actuation with steady jets doubles the achievable lift which is supportive for reliability arguments concerning the possible failure mode of clogged feedback channels.

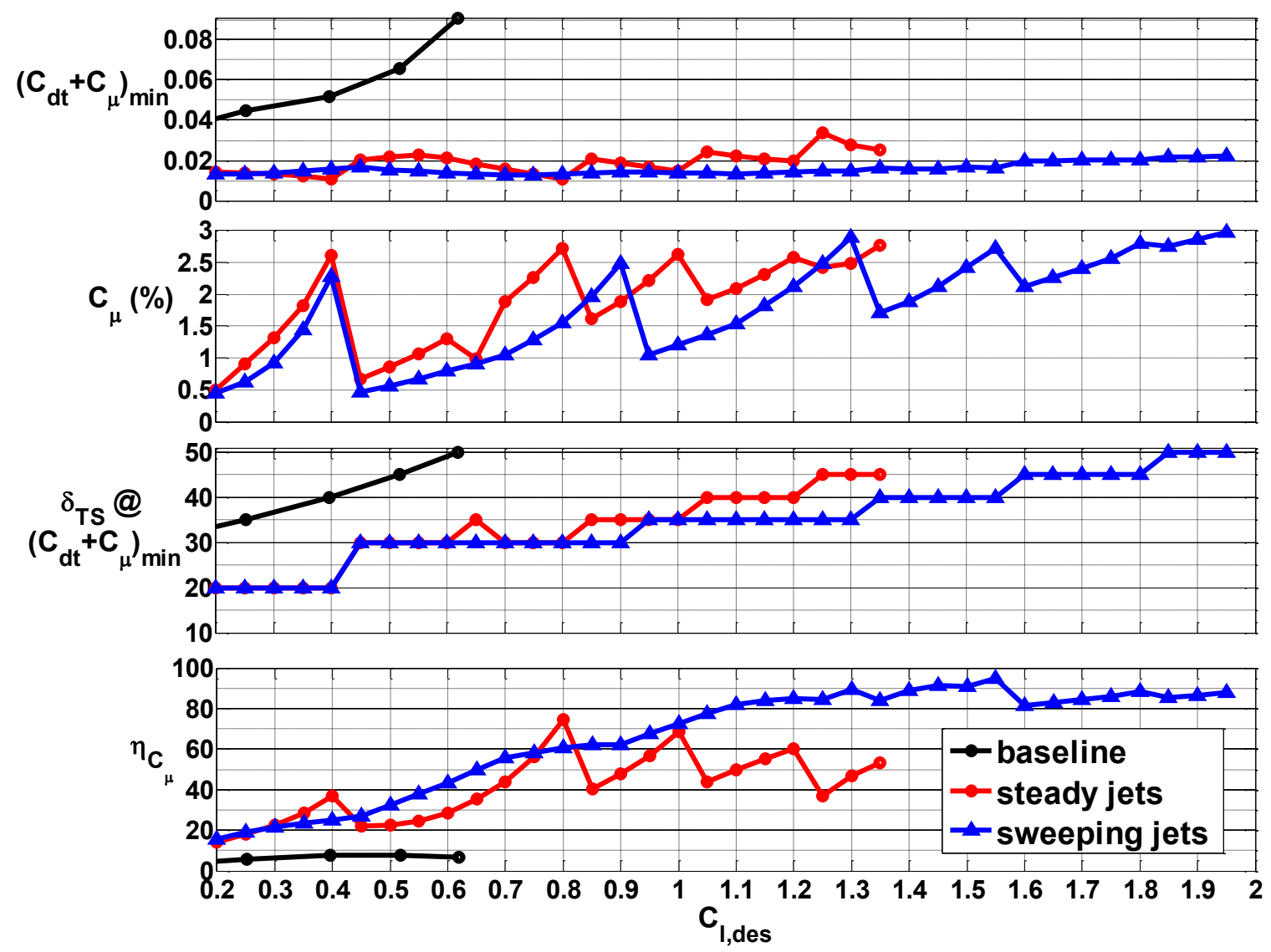

Figure 24 - Flap 20A, sweeping vs. non-sweeping jets with $\left(C_{d t}+C_{\mu}\right)_{\min }$, AFC @ 20\% $c_{f l a p}, \Delta z=0.5 \mathrm{in}$. 
Both actuation methods resemble a somewhat discontinuous pattern in corrected drag, momentum input, and $\eta_{C_{\mu}}$ with increasing lift. These discontinuities are consistent with the increase in flap deflection. At a constant $\delta_{T S}$ an increase in $C_{l, d e s}$ requires a higher momentum input to obtain this value. Once the flow is fully attached and further improvements are limited at the particular flap deflection, $\delta_{T S}$ increases to a higher value which in turn yields a sufficient lift at a smaller $C_{\mu}$. However, the larger flap deflection also causes an increase in drag. Due to the limited effectiveness of non-sweeping jets, the jumps in $\delta_{T S}$ occur at smaller $C_{l, d e s}$ than for sweeping jets. Note, that the maximum $\delta_{T S}$ for non-sweeping jets is $45^{\circ}$, whereas sweeping jets are able to attach the flow at $\delta_{T S}=50^{\circ}$, thereby yielding additional improvements in lift.

Similar observations regarding efficiency are evident when correcting the drag by the input power coefficient, $C_{E}$, instead of $C_{\mu}$ (Figure 25). The according lift-to-drag ratios obtained with the corrected drag are defined by the power efficiency, $\eta_{C_{E}}$ (Equation (11)). Because $C_{E}$ not only accounts for the inserted momentum, but also the respective velocity ratio (see Equation (10)), the results are not as optimistic as for $\eta_{C_{\mu}}$. The minimal corrected drag is found at higher flap deflections because a desired lift is achieved at smaller $C_{\mu}$ and therefore smaller $C_{E}$. Due to accounting for the expense of actuation at a higher cost, the corrected drag values yield smaller values of $\eta_{C_{E}}$ than for $\eta_{C_{\mu}}$. Note that the discontinuities in $\left(C_{d t}+C_{E}\right), C_{\mu}$, and $\eta_{C_{E}}$ are again consistent with the increases in $\delta_{T S}$, which are explained by the same reasoning as for Figure 24 . 


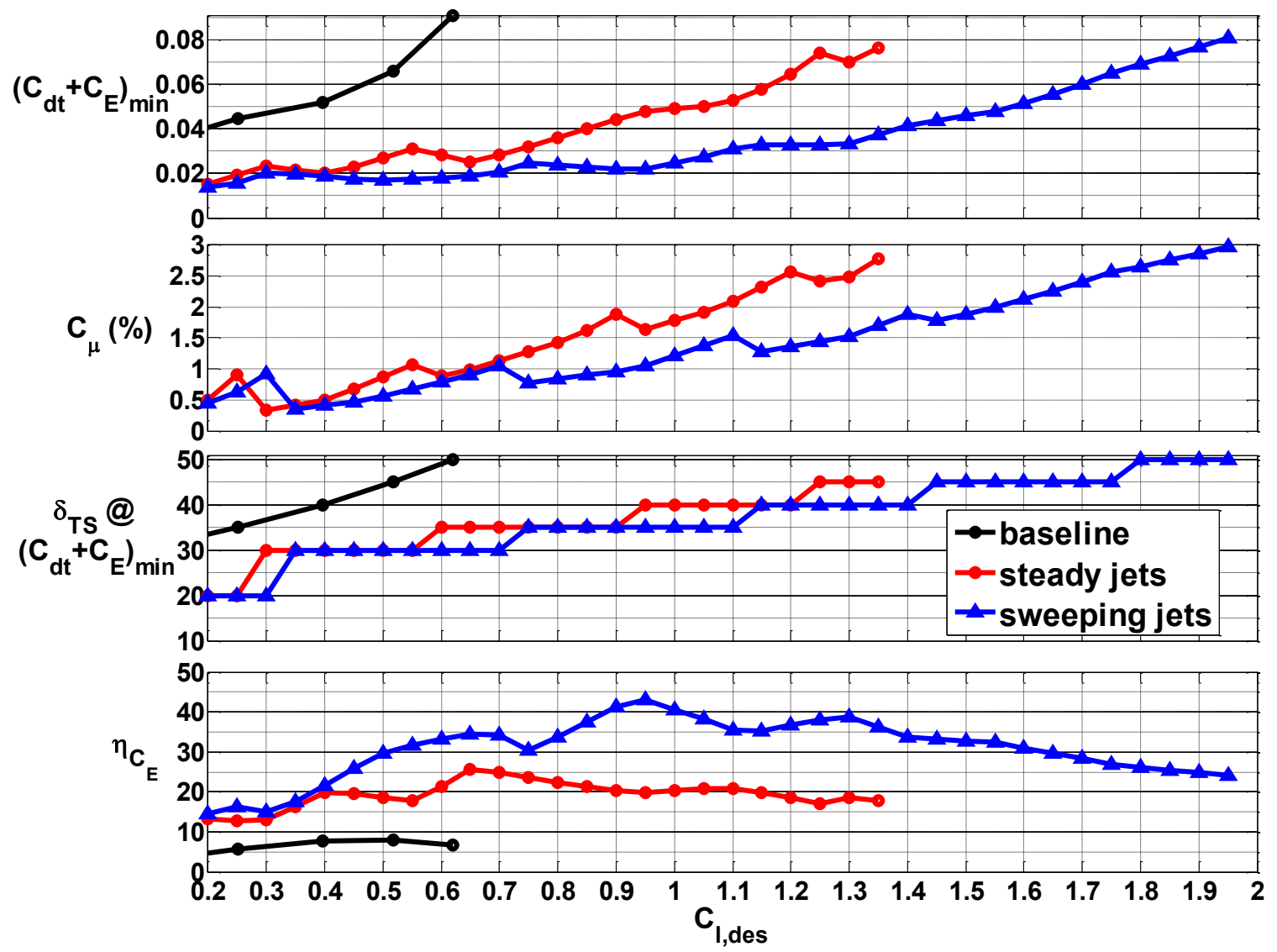

Figure 25 - Flap 20A, sweeping vs. non-sweeping jets with $\left(C_{d t}+C_{E}\right)_{\min }$, AFC $@ \sim 20 \% c_{f l a p}, \Delta z=0.5 \mathrm{in}$.

The efficiency comparison between sweeping and non-sweeping jets is summarized in Table 2 which lists the maximum momentum and power efficiency from the entire range of lift coefficients and for various actuator spacings. In addition, the maximum figure of merit is listed which defines the ratio between maximum corrected $L / D$ (i.e., $\eta_{C_{\mu}}$ or $\eta_{C_{E}}$ ) and maximum baseline $L / D$ (Equation (12)). Table 2 also indicates the lift at which these maximum values are achieved. By these numbers it is eminent that separation control with sweeping jets is far superior to non-sweeping jets. Not only do sweeping jets yield higher levels of efficiency, they also achieve these levels at a significantly higher lift. It is evident that the measure of efficiency based only on $C_{\mu}$ is far 
more optimistic than based on $C_{E}$. However, even if $\eta_{C_{E}}$ is considered as the more realistic efficiency parameter, substantial improvements are achieved compared to the baseline. However, only for $\Delta z=0.5 \mathrm{in}$. do non-sweeping jets provide valuable improvements. As the spacing increases their performance reduces to levels comparable to the baseline. Although both actuation methods achieve their highest efficiency at $\Delta z=0.5$ in., the criteria for an efficient performance strongly depends on the specific application. Limitations in available flowrate and requirements in $C_{l, \max }$ may identify a larger spacing between sweeping jets as more efficient because of the greater effectiveness in attaching the flow and achieving a higher lift.

Table 2 - Flap 20A, maximum efficiency comparison, sweeping vs. non-sweeping jets.

\begin{tabular}{|c|c|c|c|c|c|c|c|}
\hline & \multirow{2}{*}{\begin{tabular}{|c|} 
baseline \\
$(L / D)_{\max }$ \\
$C_{1}$
\end{tabular}} & \multicolumn{3}{|c|}{$\eta_{c_{\mu, \max }}\left(\mathrm{FM}_{\max }, \mathrm{C}_{\mathrm{l}}\right)$} & \multicolumn{3}{|c|}{$\eta_{\mathrm{CE}, \max }\left(\mathrm{FM}_{\max }, \mathrm{C}_{\mathrm{l}}\right)$} \\
\hline & & $\Delta \mathrm{z}=0.5 \mathrm{in}$ & $\Delta z=1$ in. & $\Delta z=1.5$ in. & $\Delta z=0.5 \mathrm{in}$ & $\Delta z=1$ in & $\Delta z=1.5$ in. \\
\hline sweeping jets & $8,0.5$ & $95(12.1,1.55)$ & $75(9.3,1.65)$ & $31(4.1,0.8)$ & $43(5.5,0.95)$ & $25(3.1,0.95)$ & $11(1.5,0.6)$ \\
\hline non-sweeping jets & $8,0.5$ & $74(9.3,0.8)$ & $42(5.1,0.8)$ & $13(1.6,0.6)$ & $26(3.2,0.65)$ & $9(1.1,0.55)$ & $8(1,0.45)$ \\
\hline
\end{tabular}

Additional evaluations of efficiency are discussed in Chapter 6 when comparing various geometric configurations.

\subsubsection{Qualitative Comparison}

The quantitative discussion of the previous chapter is complemented by a qualitative comparison to identify why sweeping jets are superior to non-sweeping jets in controlling flow separation. The comparison is based on similar surface flow visualization experiments as presented in Chapter 4.5. The photographs discussed in this chapter illustrate the $20 \%$ flap downstream of the actuation location at $\sim 20 \% c_{\text {flap }}$. The free-stream is directed from the top to the bottom of the pictures. Horizontal scales are 
included which also apply for the vertical direction. In order to reveal the most apparent differences, the highest available value of flowrate and momentum is chosen for each actuator spacing.
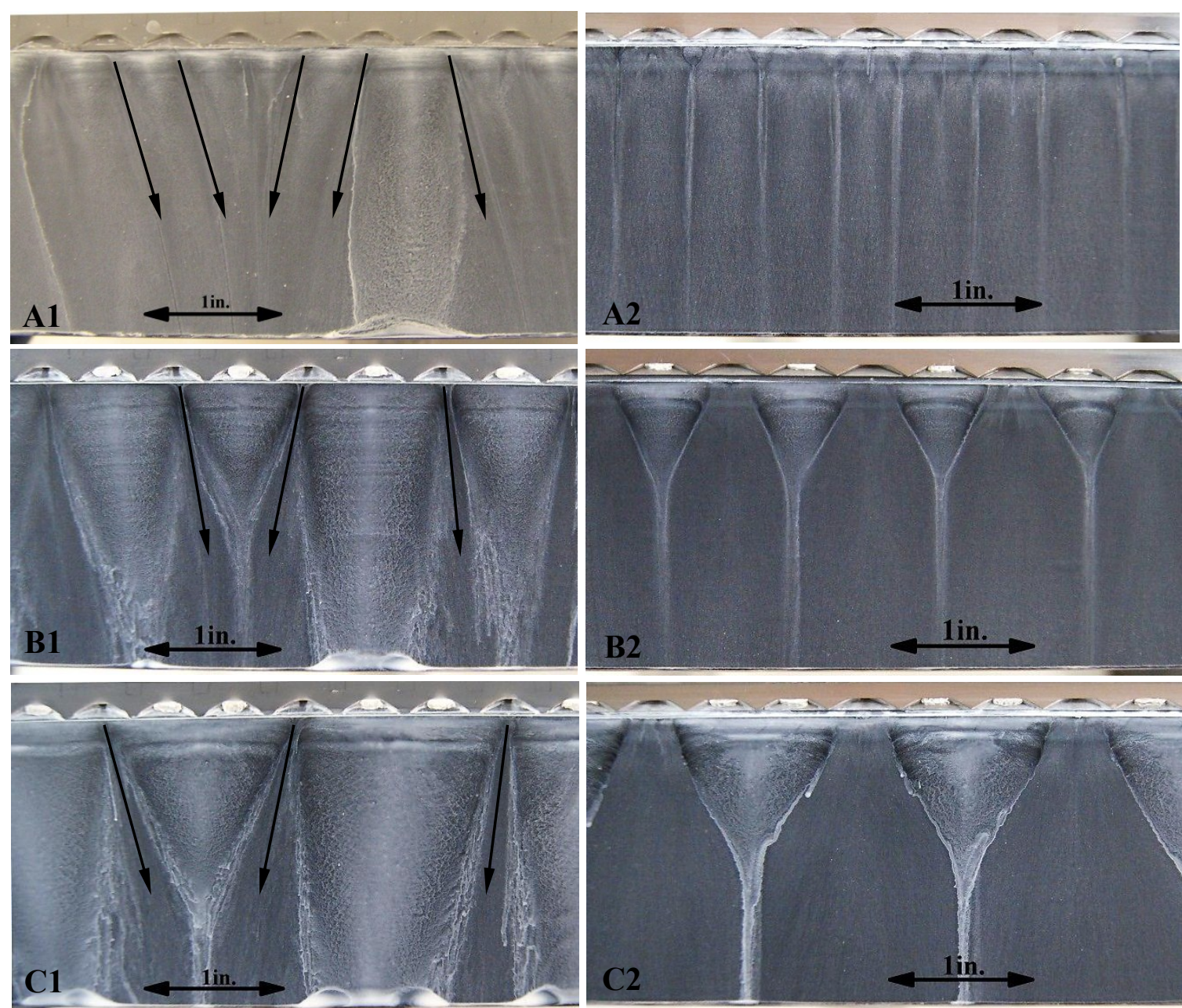

Figure 26 - Flap 20A, surface flow visualization, no free-stream, AFC @ 20\% $c_{\text {flap }}$, entire flap, left: non-sweeping jets, right: sweeping jets, A) $\Delta z=0.5 \mathrm{in} ., Q \leftrightarrow C_{\mu}=3 \%$ @ $R e=6 \cdot 10^{5}$, B) $\Delta z=1$ in., $Q \leftrightarrow C_{\mu}=6 \%$ (a) $R e=6 \cdot 10^{5}$, C) $\triangle z^{\prime}=1.5 \mathrm{in} ., Q \leftrightarrow C_{\mu}=4.5 \% @ R e=6 \cdot 10^{5}$.

The differences between sweeping and non-sweeping jets are first discussed in absence of a free-stream for all actuator spacings (Figure 26). The pictures on the left illustrate non-sweeping jets, whereas the pictures on the right resemble sweeping jets. For different values of $\Delta z$ the same actuators are shown to track their individual behavior. 
Although the feedback channels are sealed, the non-sweeping jets do not exit straight from the actuator due to the bistable mode of internal flow attachment. As the jet passes through the actuator's main chamber (see Figure 1), it attaches to either side and remains there. Therefore, the jet is emitted at an angle. Whether the jet exits to the right or left is dependent on small variations in each actuator's geometry. As depicted in Figure 26 (left), the exit direction does not change with flowrate or actuator spacing. This random behavior yields different size areas along the span which are not covered by the jets. Therefore, significant portions of the flap may not be directly impacted by non-sweeping jets especially for large dz. In contrast, sweeping jets provide large area coverage due to their sweeping motion and show a homogeneous and repetitive pattern along the span. Especially for $\Delta z=1.5 \mathrm{in}$., significant differences in area coverage are evident. However, even for sweeping jets with $\Delta z=1.5 \mathrm{in}$. the area not directly reached by the jets' sweeping motion resembles a significant portion of the total flap size. The size of the area not covered by the jets depends on the sweeping angle and the distance between adjacent actuators. For $\Delta z=0.5 \mathrm{in}$. the jets intercept close to the exit, whereas for $\Delta z=1.5 \mathrm{in}$. this point moves substantially downstream (Figure 26, right). For these particular actuators, the sweeping angle does not appear to change with flowrate per actuator and therefore, the location of where the jets' intercepts is only dependent on $\Delta z$.

The inherent differences between sweeping and non-sweeping jets are also apparent in the presence of a free-stream (Figure 27) with the flap's top surface being deflected by $40^{\circ}$. The photographs in Figure 27 illustrate the same section of the flap and therefore the same actuators as in Figure 26. The baseline case is omitted because the 
flow is entirely separated over the flap and the resulting surface flow visualization is similar to the picture previously shown in Figure 17. Although non-sweeping jets spaced at $\Delta z=0.5 \mathrm{in}$. are capable of controlling flow separation over wide regions of the flap, some areas of accumulated fluid and even reversed flow are evident. These areas increase in size and move further upstream with increasing actuator spacing. For $\Delta z=1.5 \mathrm{in}$. (Figure 27, C1) convincing evidence for reversed and separated flow is depicted.
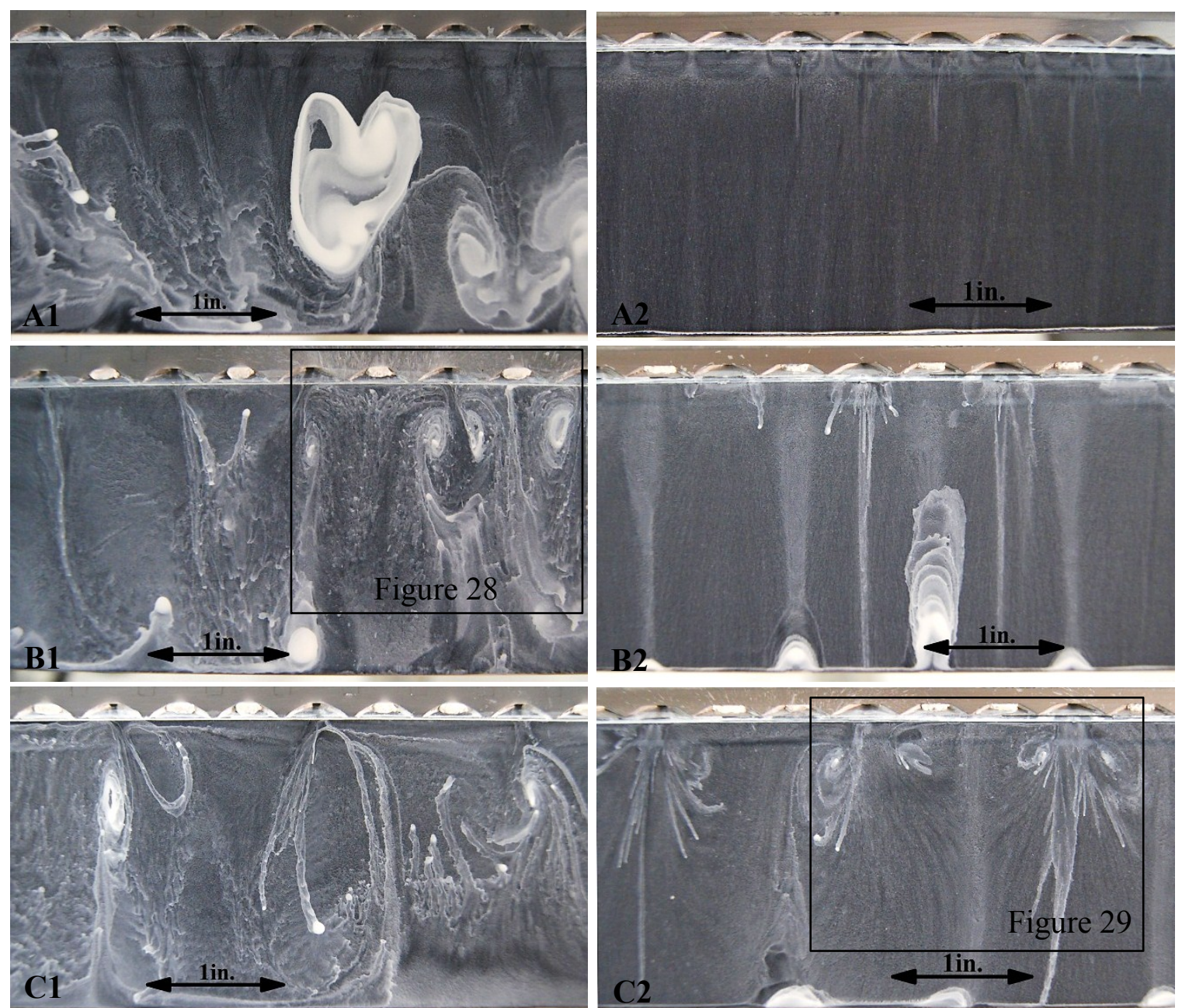

Figure 27 - Flap 20A, surface flow visualization, $\alpha=0^{\circ}, \delta_{T S}=40^{\circ}$, AFC @ 20\% $c_{\text {flap }}$, entire flap, left: non-sweeping jets, right: sweeping jets, A) $\Delta z=0.5$ in., $C_{\mu}=3 \%$, B) $\Delta z=1$ in., $C_{\mu}=6 \%$, C) $\Delta z=1.5$ in., $C_{\mu}=4.5 \%$. 
Even at first glance, it is apparent that sweeping jets yield cleaner pictures with less accumulated fluid and more organized patterns (Figure 27, right). For $\Delta z=0.5 \mathrm{in}$. the flow is completely attached over the flap. Streamwise patterns in between adjacent actuators indicate the surface traces of streamwise vortices as suggested in Chapter 4.5. These traces are evident for all actuator spacings. However, the onset of the streamwise vortices only becomes evident for larger $\Delta z$, because more space is available for these structures to manifest themselves. Similar to the findings discussed in Chapter 4.5, a wavy pattern of accumulated fluid is noticeable at the flap's trailing edge which marks the departure of the pairs of streamwise vortices away from the surface.

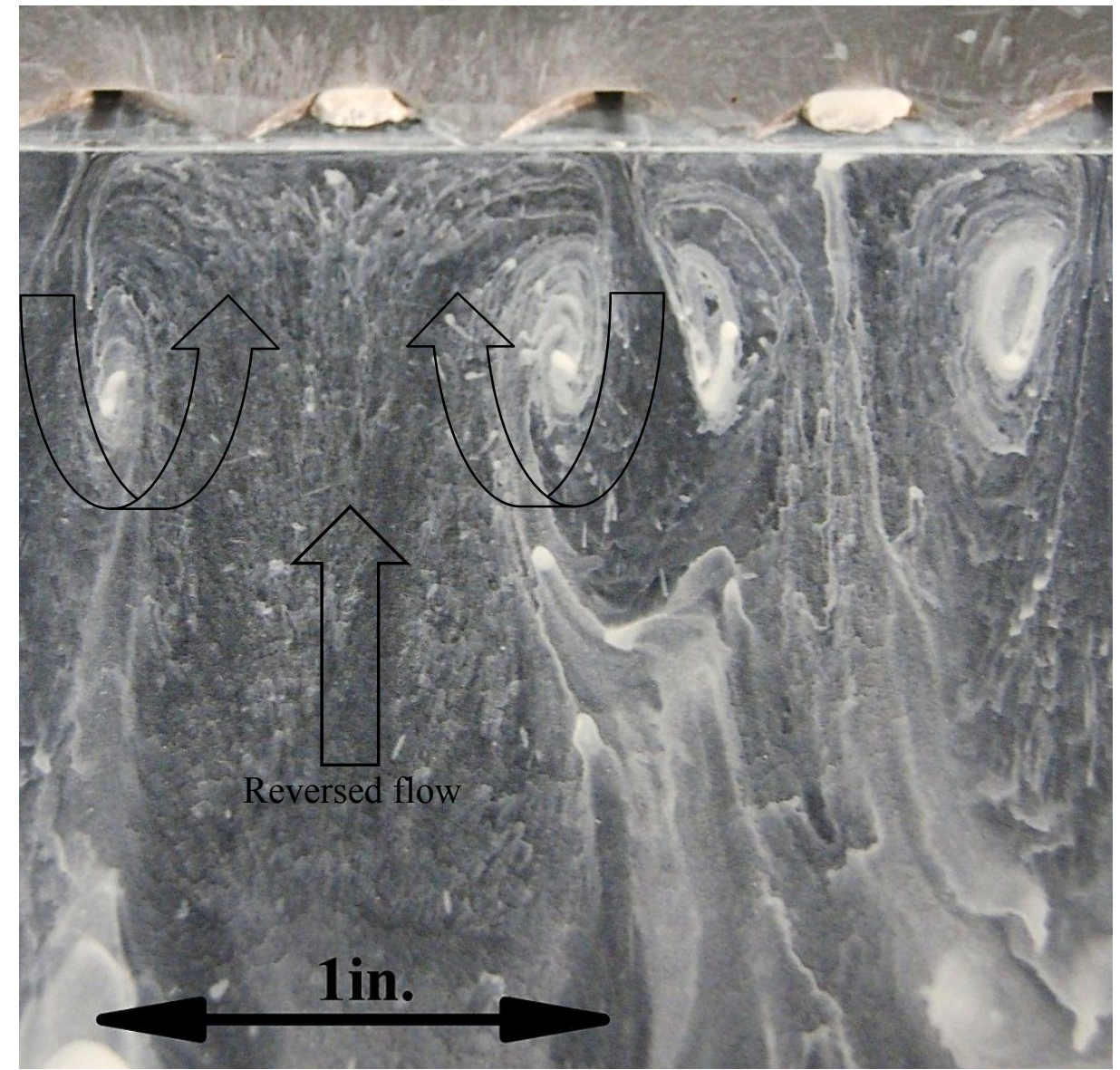

Figure 28 - Flap 20A, surface flow visualization, $\alpha=0^{\circ}, \delta_{T S}=40^{\circ}$, AFC with non-sweeping jets $@ \sim 20 \%$ $c_{\text {flap }}, \Delta z=1$ in., $C_{\mu}=6 \%$, close-up view of actuator outlet. 
Additional details are provided by enlarging some areas of interest from Figure 27. First, the region downstream of actuators spaced at lin. is enlarged for non-sweeping jets (Figure 28). Each jet appears to form a pair of normal vortices which is eventually bent by the free-stream to form a pair of streamwise vortices as suggested by Kumar ${ }^{[12]}$, who studied this behavior in conjunction with microjets. In between adjacent actuators, the normal vorticity causes an upstream flow which would counteract the intention to control flow separation. Since the visualizing fluid was only applied downstream of the actuators, the spray of fluid visible upstream of the actuators also indicates reversed flow. Therefore, the effectiveness of non-sweeping jets is significantly reduced as the distance between them increases. For sweeping jets spaced at $\Delta z=1$ in. no evidence is found for this behavior. However, for $\Delta z=1.5$ in. some indication of reversed flow is noticeable from minor splashes of fluid upstream of the actuators (Figure 29). This also supports the previous argument that the spanwise spacing between actuators is limited for sweeping jets to be effective. Nevertheless, these observations are restricted to the vicinity of the actuators. Farther downstream, the effect of the neighboring jet inhibits any reversed flow. Similar to non-sweeping jets, normal vortices are formed at the edge of the jets' sweeping range which are bent in streamwise direction to form pairs of streamwise vortices. These vortices push fluid toward the center between adjacent actuators where it is moved downstream by the sweeping jets and the free-stream. These observations are consistent with the discussion presented in Chapter 4.5. 


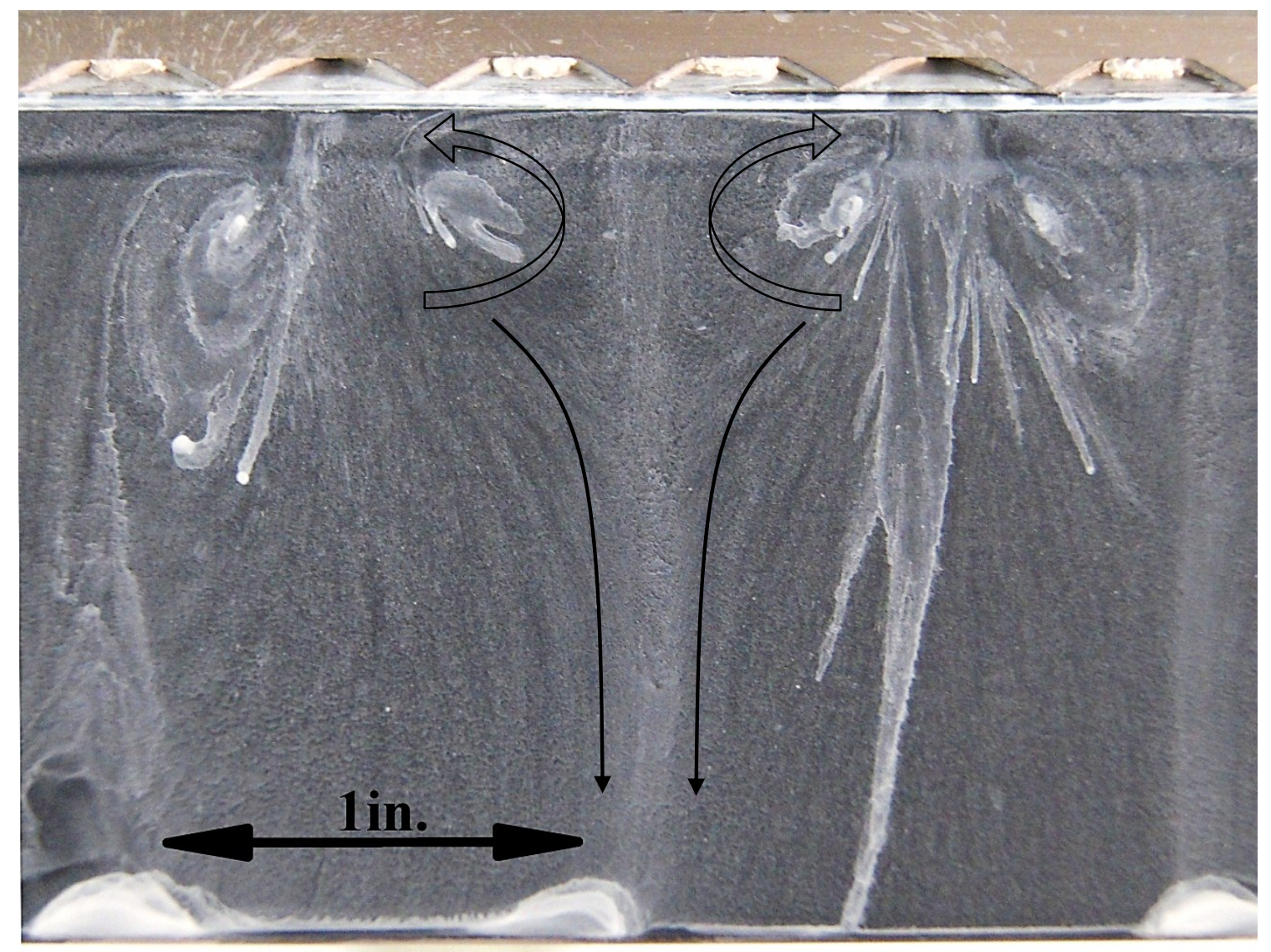

Figure 29 - Flap 20A, surface flow visualization, $\alpha=0^{\circ}, \delta_{T S}=40^{\circ}$, AFC with sweeping jets $@ \sim 20 \% c_{f l a p}$, $\Delta z=1.5$ in., $C_{\mu}=4.5 \%$, entire flap.

Although both sweeping and non-sweeping jets appear to inherit their potential to control separation from streamwise vortices that they form, sweeping jets institute their superior performance through the increased area coverage and the interaction between adjacent jets. These properties allow sweeping jets to be spaced at larger distances and therefore, the total flowrate can be reduced. A comparison between steady jets, exiting in a straight direction from an orifice, and sweeping jets may be warranted to irrefutably prove the superior performance of sweeping jets. 


\section{WHERE SHOULD ACTUATION BE APPLIED?}

The previous chapters discuss results for actuation from particular locations without justifying the choice of location. The following subsections address observations regarding actuation from a single row of actuators at various locations and simultaneous actuation from multiple rows of actuators.

\subsection{Actuation from a Single Location}

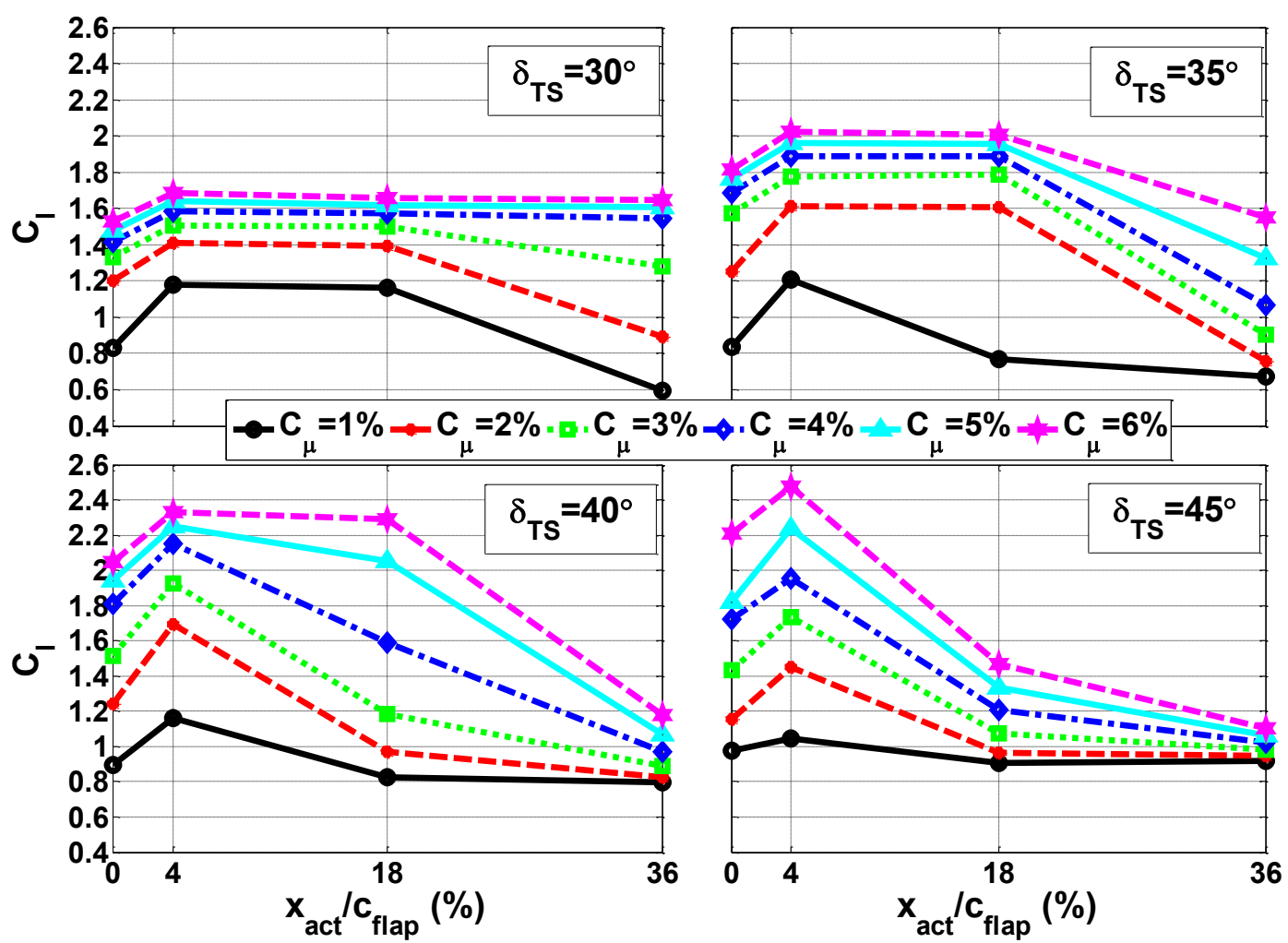

Figure 30 - Flap 40B, lift vs. actuation location, $\alpha=0^{\circ}, \Delta z=1 \mathrm{in}$.

Various actuation locations were tested for different flap deflections and flap sizes. Although the resolution in actuation location is coarse, some dominant trends and observations are revealed. Figure 30 illustrates the lift as a function of actuation location for various flap deflections for $\alpha=0^{\circ}$ and $\Delta z=1$ in. This spacing is the most effective for a 
wide range of $C_{\mu}$. For small $\delta_{T S}$ only minor differences between the various locations are evident because the flow is easily attached at low momentum from any location. As the flap's deflection increases actuation from $4 \% \quad c_{\text {flap }}$ emerges as being superior. Discrepancies are noted with actuation from the flap shoulder which are addressed shortly. Since none of the lines for various $C_{\mu}$ are intersecting at any $\delta_{T S}$, it can be concluded that the ideal actuation location is independent of flap deflection and momentum input, at least within the coarse range of locations tested. Nagib et al. ${ }^{[7]}$ indicate that for conventional active flow control methods the actuation location is dependent on flap deflection since the location changes as the flap is deflected. If this claim applies to separation control with sweeping jets remains to be verified by a finer resolution in actuation location. If the flow is fully attached, all locations on the flap yield comparable lift values. However, the further actuation is applied downstream on the flap, the higher the momentum necessary to attach the flow. Therefore, it may be stated that the achievable lift at a certain flap deflection is independent of actuation location; however, it is strongly dependent on actuation intensity. Note that once the flow over the flap is fully attached the additional improvements in lift are marginal. This discussion is continued in more detail in Chapter 6.1.

The trends regarding actuation location are unchanged when considering different distances between adjacent actuators (Figure 31). Applying actuation on the flap close to the flap's leading edge is most effective for all $\Delta z$. However, the differences between the various actuation locations are more pronounced for $\Delta z=0.5 \mathrm{in}$ and $1.5 \mathrm{in}$., independent of whether $C_{\mu}$ or $C_{Q}$ is considered as the comparing parameter. This implies that the 
effectiveness in controlling flow separation over the flap becomes more sensitive to actuation location the more actuation is applied at its non-optimal configuration (i.e., actuator spacing and size). This observation may be advantageous for scaling and optimization processes because without optimizing the actuator configuration, differences in performance of various actuation locations can be more easily detected.
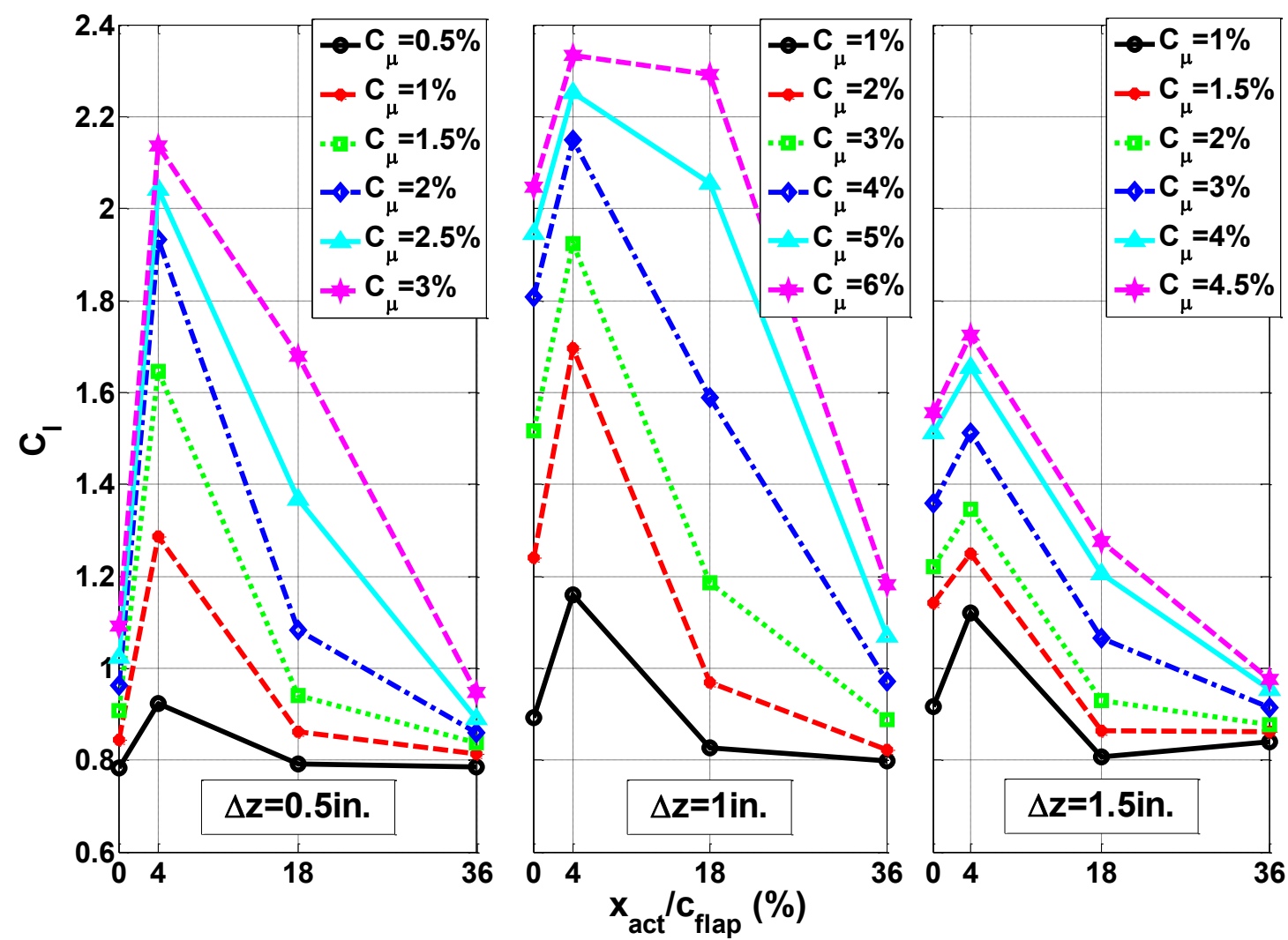

Figure 31 - Flap 40B, lift vs. actuation location, $\alpha=0^{\circ}, \delta_{T S}=40^{\circ}$.

The statement that actuation is most effective when applied from the flap and close to its leading edge is verified for all flap geometries and sizes tested (Figure 32). However, it appears that actuation from locations farther downstream becomes more effective as the flap size decreases. Since the various flap sizes inherit different geometric properties, the comparison is made in reference to the performance of the flap shoulder. 
Considering the two extreme cases of a $60 \%$ flap and a $20 \%$ flap reveals that for the $60 \%$ flap actuation from $18 \% c_{\text {flap }}$ is ineffective in controlling separation when compared to the flap shoulder, whereas actuation from $22 \% c_{\text {flap }}$ on the $20 \%$ flap is superior to actuation from the flap shoulder. This implies that referencing actuation location as a percentage of the flap's chord is not appropriate. Instead an absolute distance on the flap normalized by a common reference (e.g., the total chord length, $c$ ) appears more suitable. For example, comparing the $12 \%, 18 \%$, and $40 \% c_{\text {flap }}$ location on the $60 \%, 40 \%$, and $20 \%$ flap, respectively, indicates a similar performance in reference to the flap shoulder. As shown in Figure 32, all these locations have the same total distance from the shoulder (i.e., $7.2 \% c$ ).
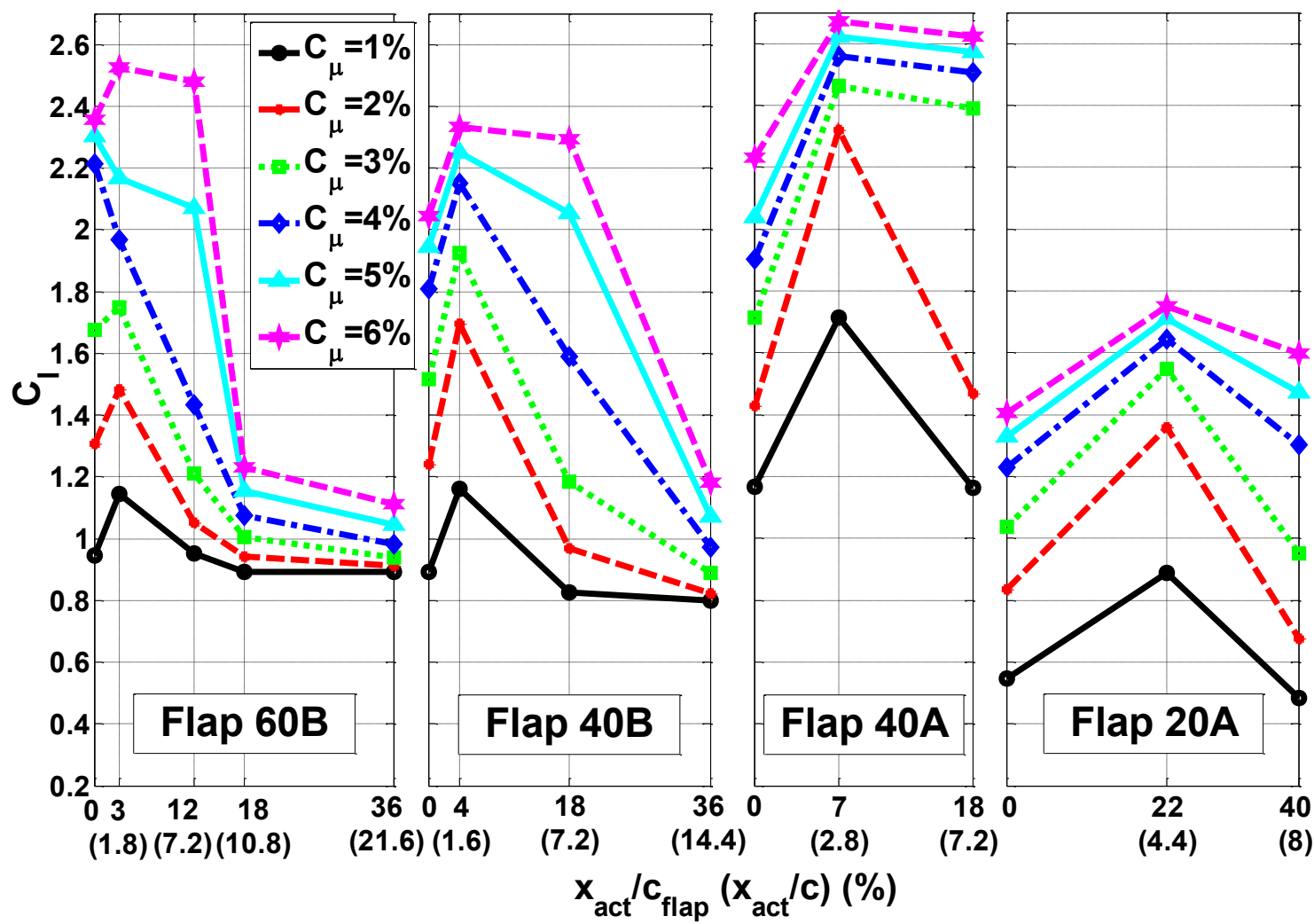

Figure 32 - All flaps, lift vs. actuation location, $\alpha=0^{\circ}, \delta_{T S}=40^{\circ}, \Delta z=1$ in. 
For the use of microjets, Kumar ${ }^{[12]}$ indicated that the jets' penetration depth to the separation shear layer affects the ideal actuation location which is dependent on the jets' exit angle, actuator size, and velocity ratio. Therefore, other actuator configurations may yield a different optimal actuation location and/or enhanced performance in controlling flow separation. Unfortunately, the resolution in actuation location and flap size in the current study is insufficient to pinpoint these trends more precisely.

As mentioned before, discrepancies are evident regarding actuation from the flap shoulder. The observed trend that actuation is most effective the closer it is applied to the leading edge of the flap would suggest that actuation from the flap shoulder is most effective. However, this is not the case. Several reasons may explain the reduced effectiveness of this location. First, the location itself may not be ideal for controlling separation over the flap. As indicated by Nagib et al. ${ }^{[7]}$ and $\mathrm{Chen}^{[9]}$, the ideal location for continuous blowing to be effective is downstream of the separation location. Although the comparison between sweeping jets and continuous blowing may not hold significant merit, it is suggested in the previous paragraph that actuation applied a certain distance downstream of the separation location (i.e., the flap shoulder) is most effective in controlling separation. Since the flap shoulder either coincides with the separation location or is even upstream of the separation location at smaller flap deflections, the effectiveness of actuation from this location may be reduced. 

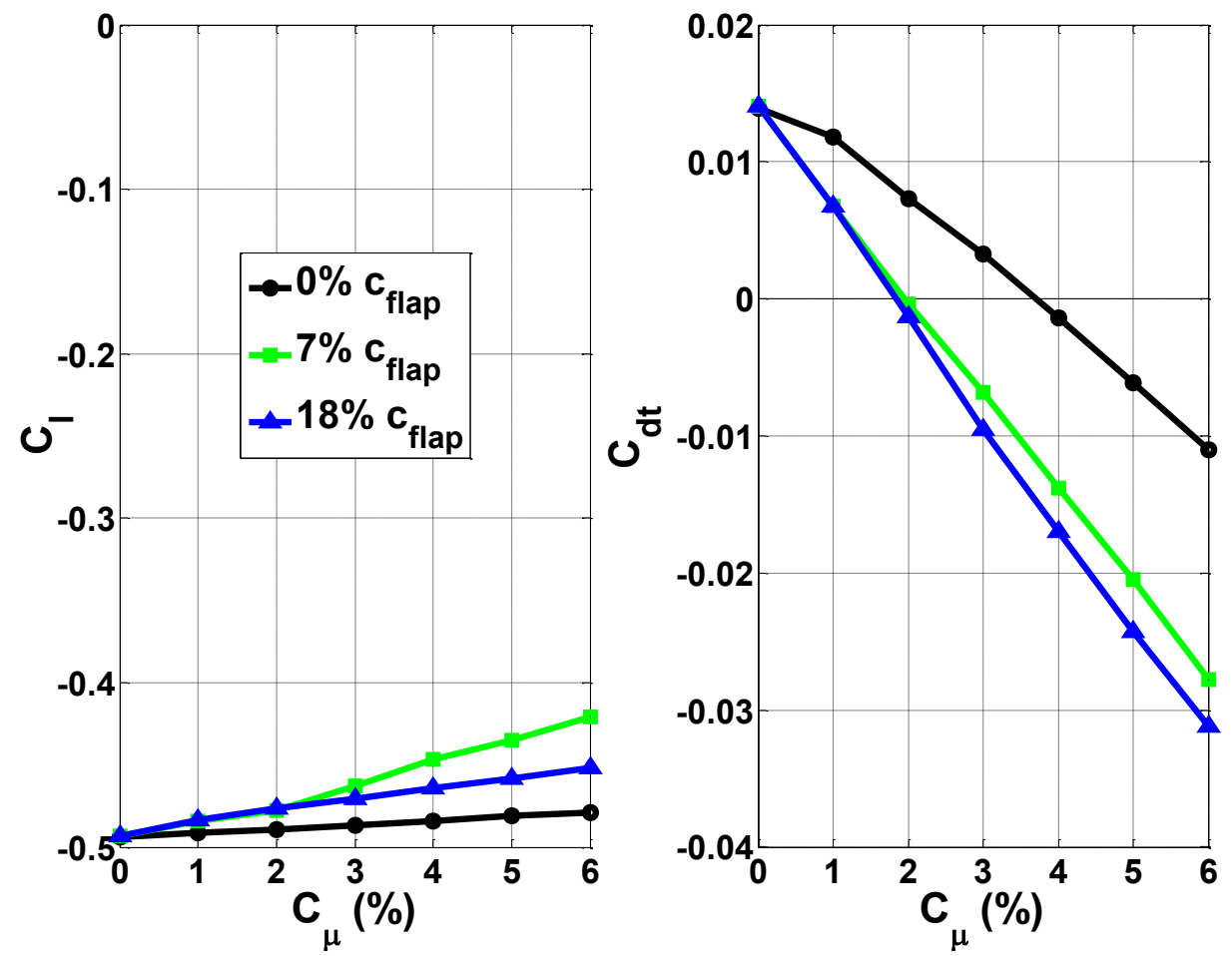

Figure 33 - Flap $40 \mathrm{~A}$, lift $\&$ total drag vs. $C_{\mu}, \alpha=0^{\circ}, \delta_{T S}=0^{\circ}, \Delta z=1$ in.

Further, more obvious explanations for the discrepancies may be based on geometric differences. The jets' exit angle of $30^{\circ}$ is referenced to the local surface which, in case of the flap shoulder, is the main element's top surface. Therefore, the angle between the jets and the flap's top surface is increasing as the flap is deflected. Another major difference to the locations on the flap is the downstream curvature of the flap's leading edge. Depending on $\delta_{T S}$, at least $30^{\circ}$ of curvature has to be overcome to meet the straight top surface of the flap. Although both effects are coupled, there is evidence that the curvature plays a more significant role. If the exit angle were to be the major reason, it would be expected that actuation from the flap shoulder becomes less effective as the flap deflection is increased. However, Figure 30 does not suggest any additional loss in effectiveness as $\delta_{T S}$ increases, even though at $\delta_{T S}=45^{\circ}$ the angle between the jets emitted 
from the flap shoulder and the flap's top surface is equal to $75^{\circ}$. This also implies that exit angles significantly larger than $30^{\circ}$ may be of interest and should be investigated in future experiments. In fact, Kumar et al. ${ }^{[12]}$ suggest that exit angles around $90^{\circ}$ are most effective for separation control with microjets. In order to decouple the effects of exit angle and curvature, the case of $\delta_{T S}=0^{\circ}$ is discussed for which the jets' exit angle is the same for all locations (Figure 33). For the $40 \%$ simple flap configuration with $\delta_{T S}=0^{\circ}$ the flow is fully attached around the airfoil. Due to the negative camber the airfoil yields a negative lift (Figure 33). The local acceleration of the flow increases the lift when applying actuation which is depicted in the $C_{p}$-distributions in Figure 34 (left). Actuation from $7 \% c_{\text {flap }}$ yields slightly more lift improvement than the $18 \% c_{\text {flap }}$ location because of its larger upstream effect. By this explanation actuation from the flap shoulder should provide the highest gain in lift which is not the case. Note that the gap between the main element and the flap is sealed internally and externally at the model's bottom surface. Thus, the only difference between the three locations is the downstream curvature. Since the changes in lift are marginal and the resolution in pressure distribution insufficient in the vicinity of the actuation location, the discussion based on lift may not be conclusive. However, the arguments become more convincing when considering the total drag. The drag reductions obtained from inserting momentum from the flap are comparable. The $18 \% c_{\text {flap }}$ location provides slightly smaller drag due to its shorter distance to the trailing edge and therefore, smaller skin friction losses, which is evident in the wake profiles shown in Figure 34 (right). The recovery of momentum when inserted from the flap shoulder is significantly less than from the other locations which cannot be explained by 
the additional skin friction losses. The respective wake (or jet) profile is shallower and its center is shifted further upwards than with actuation from the flap. This also implies that the jets, departing the flap's trailing edge, are further away from the surface and may not even be attached to it. Further support for this claim is noticed at the lower end of the velocity profile where remnants of a velocity deficit are evident. Despite the close proximity and the same exit angle for all three locations, the different behavior of actuation from the flap shoulder leads to the conclusion that the downstream curvature is the main cause for the reduced effectiveness of this location.
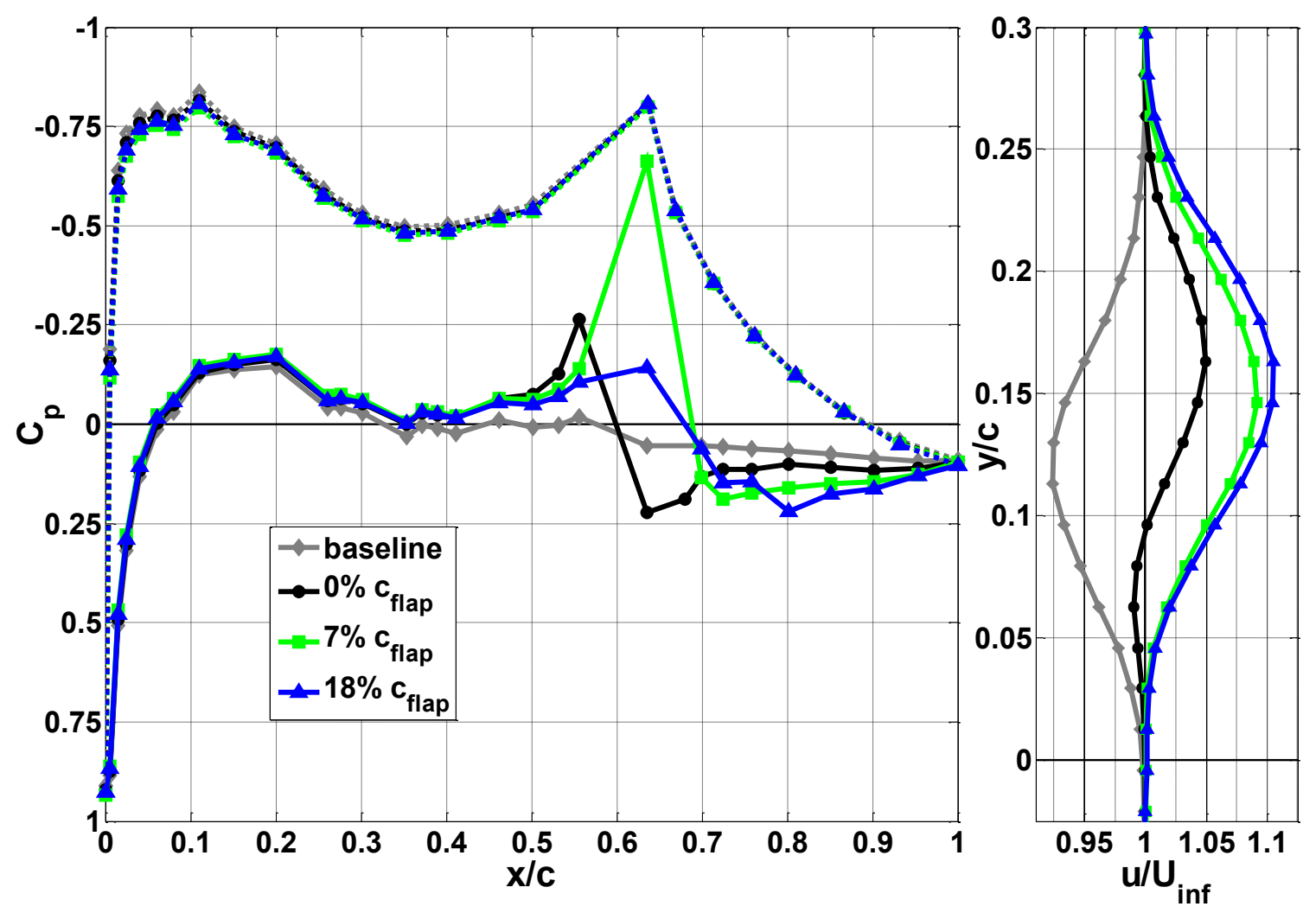

Figure 34 - Flap 40A, $C_{p}$-distributions \& wake profiles, $\delta_{T S}=0^{\circ}, \alpha=0^{\circ}, C_{\mu}=6 \%, \Delta z=1$ in.

The observation that actuation from the flap is most effective the closer it is applied to the flap's leading edge, can be summarized by a single coefficient. Figure 35 
illustrates that the coefficient $C_{\mu} \cdot\left(100-x_{a c t} / c_{f l a p}\right)^{0.5}$ collapses the lift curves from various locations once the flow is fully attached. It also depicts that significantly more momentum is required to attach the flow the farther downstream actuation is applied on the flap. Although this coefficient suggests that actuation from the flap shoulder should be most effective, it does not hold for this location due to the previously discussed differences to actuation from the flap. The applicability of the coefficient $C_{\mu} \cdot(100-$ $\left.x_{a c t} / c_{f l a p}\right)^{0.5}$ is verified for different flap sizes as well (not shown).
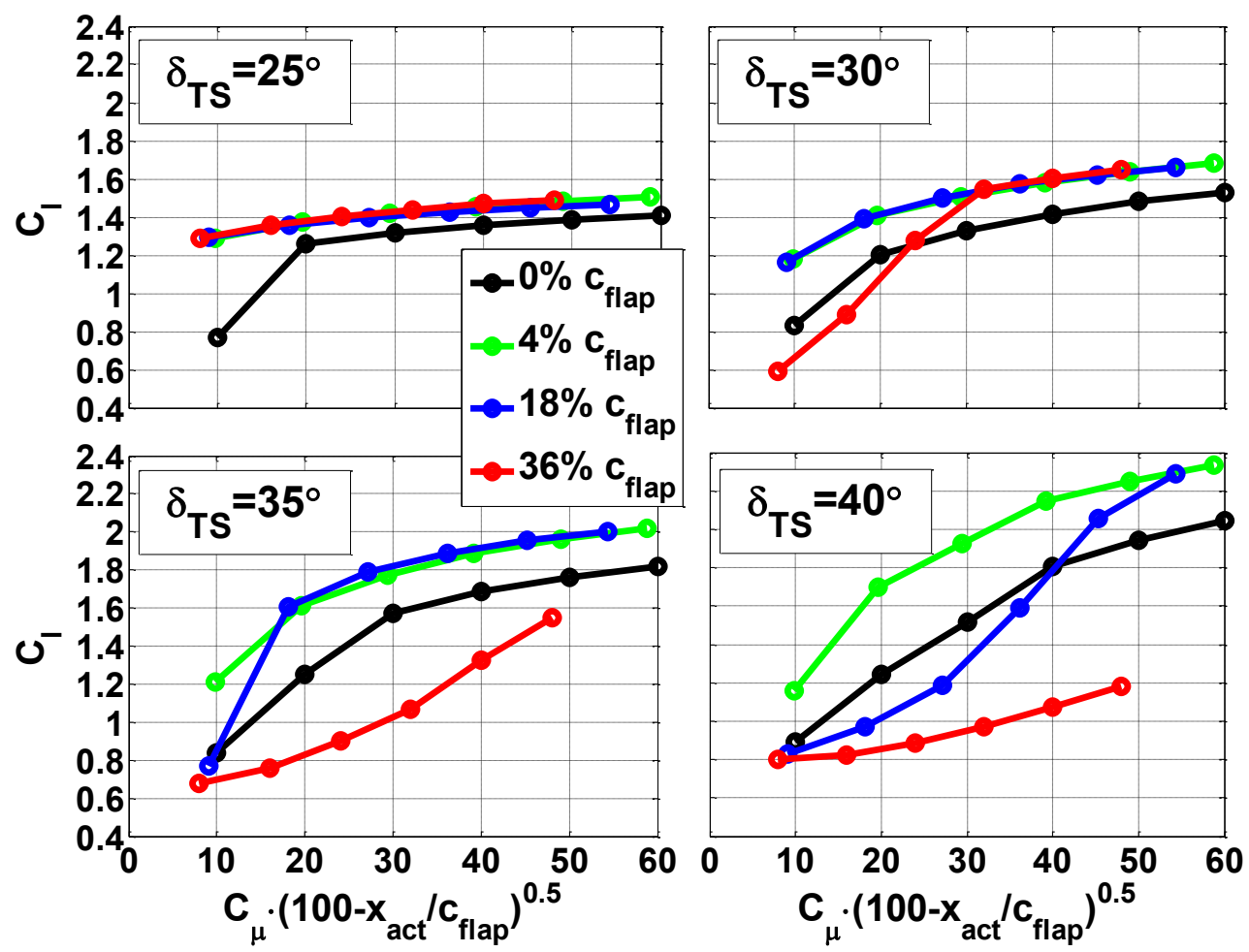

Figure 35 - Flap 40B, lift vs. $C_{\mu}\left(100-x_{a c} / c_{f l a p}\right)^{0.5}, \alpha=0^{\circ}, \Delta z=1$ in.

The discussion of parameters affecting actuation location generates some uncertainty and leaves many unanswered questions. Therefore, a more detailed investigation targeting the dependence of actuation location on flap deflection, curvature, exit angle, actuator size, and velocity ratio is warranted. Since the current study is 
focused on separation control over a deflected flap, the delay of stall over an airfoil's main element requires a different optimized actuation location which may be affected by similar parameters (e.g., exit angle and downstream curvature).

\subsection{Actuation from Multiple Locations}

Besides the investigation of a single row of actuators, various combinations of simultaneous actuation from two rows of actuators were tested on the flap configuration 40B. An exemplary portion of this data set is shown in Figure 36 for $\Delta z=1$ in. and at $\delta_{T S}=40^{\circ}$ which inherits strongly separated flow over the flap. The most effective locations (i.e., $0 \%$ and $4 \% c_{\text {flap }}$ ) are simultaneously actuated in conjunction with other locations. Note that the actuators located at the flap shoulder are shifted in $z$-direction compared to the actuators on the flap whereas the actuators in all rows on the flap are aligned. Independent of which combination of rows is actuated, the achieved lift is comparable to actuation from a single row at $4 \% c_{\text {flap }}$ when considering the same momentum input (Figure 36, left). Note that single row actuation from $36 \% c_{\text {flap }}$ becomes significantly more effective if actuation from $4 \% c_{\text {flap }}$ is added. In order to actuate with the same $C_{\mu}$ simultaneous actuation from two rows requires $41 \%$ more total flowrate into the system. Therefore, actuation from a single row is more effective when considering the required flowrate (Figure 36, right). Although a higher flowrate is necessary to obtain improvements similar to a single location, this result implies that a reduced velocity ratio can still be effective in conjunction with an increased flowrate. With two rows of actuators the same $C_{\mu}$ is maintained at a $29 \%$ smaller velocity ratio. This may have significant implications for scaling efforts. For instance, if the exit velocity of the jets is 
limited either by the actuator per se or by intention, the necessary values of $C_{\mu}$ have to be achieved by increasing the actuator size and the flowrate. Due to higher free-stream velocities on full-scale models, the velocity ratio may be significantly reduced which may not be detrimental if the flowrate into the system is increased accordingly. This argument is supported by the discussion in Chapter 4.5 which suggests that the formation of streamwise vortices is the underlying mechanism controlling separation. The strength of these vortices is dependent on the jets' velocity and flowrate. Even though the jets' velocity may be limited, the flowrate can be increased by enlarging the actuators to provide the necessary vortex strength.
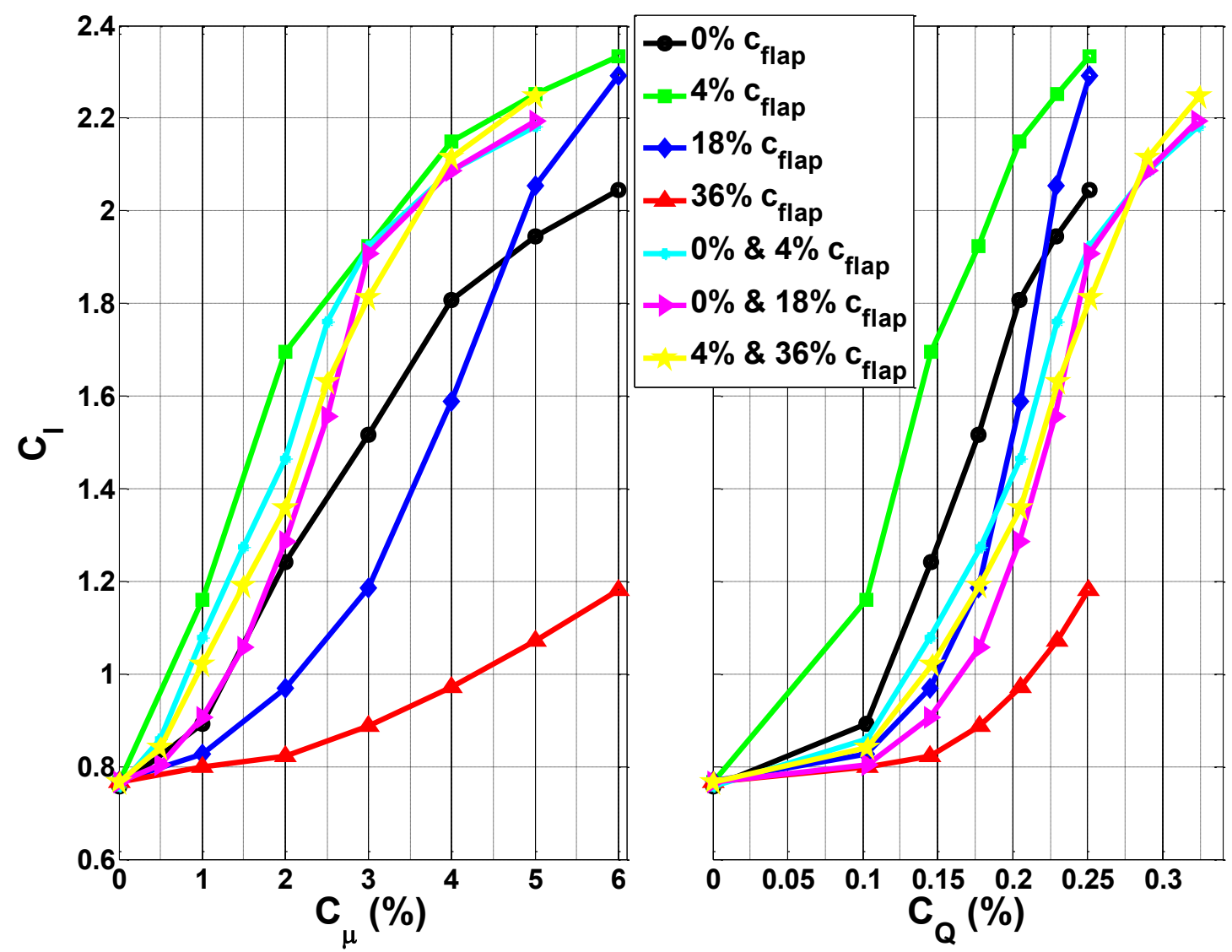

Figure 36 - Flap 40B, lift vs. $C_{\mu} \& C_{Q}, \alpha=0^{\circ}, \delta_{f}=40^{\circ}, \Delta z=1$ in. 
Although simultaneous actuation from multiple locations does not appear useful for the current setting, it may be an option for large-scale applications. The use of multiple locations provides a possibility to obtain desired values of $C_{\mu}$ or $C_{Q}$ if the increase in actuator size is either limited or unpractical. An additional row of actuators farther downstream on the flap may also enhance the overall penetration length of the sweeping jets and the streamwise vortices they form. A detailed investigation of penetration length in conjunction with flap and actuator size has not yet been undertaken. 


\section{ON WHAT AIRFOIL CONFIGURATION SHOULD ACTUATION BE APPLIED?}

The main goal of active separation control is to provide a required lift as effectively and efficiently as possible, which strongly depends on the particular airfoil configuration (i.e., flap deflection, flap size, angle of attack, and airfoil shape). This chapter addresses the effects of geometric parameters to aid the choice of an appropriate geometry. First, a detailed comparison between experimental and numerical data is presented to establish whether potential flow solutions are a useful tool to predict the achievable performance of a particular geometry. The following subsections individually discuss the effects of flap deflection, flap size, and incidence, as these parameters strongly influence the attainable lift and the respective drag. Besides further aiding the scaling of sweeping jet actuators, the presented trends are intended as basic guidelines to meet a desired performance with the most appropriate geometric configuration.

\subsection{Comparisons between Numerical and Experimental Data}

The following two sections discuss whether the achievable lift through actuation with sweeping jets can be predicted by a potential flow solution. Since sweeping jet actuators continuously emit a high velocity jet that rapidly oscillates in spanwise direction, the resulting effect on the average flow may be similar to a steady, twodimensional jet emitted from a continuous slot. Due to the jets' high velocity, it may be possible to achieve lift values which exceed inviscid flow predictions based on the jet-

flap principle ${ }^{[61-65]}$. In order to enlighten this discussion, the inviscid solutions provided 
by XFOIL ${ }^{[73]}$ are compared to experimental data at $\alpha=0^{\circ}$. Actuation from the most effective location and with $\Delta z=1$ in. is considered because this configuration yields the highest lift improvements and provides the largest range of momentum coefficients. Two flap configurations are investigated for this comparison. First, the configuration 40B with a cambered flap is discussed (Chapter 6.1.1) followed by the configuration 40A which has a symmetric flap (Chapter 6.1.2).

\subsubsection{Cambered Flap}
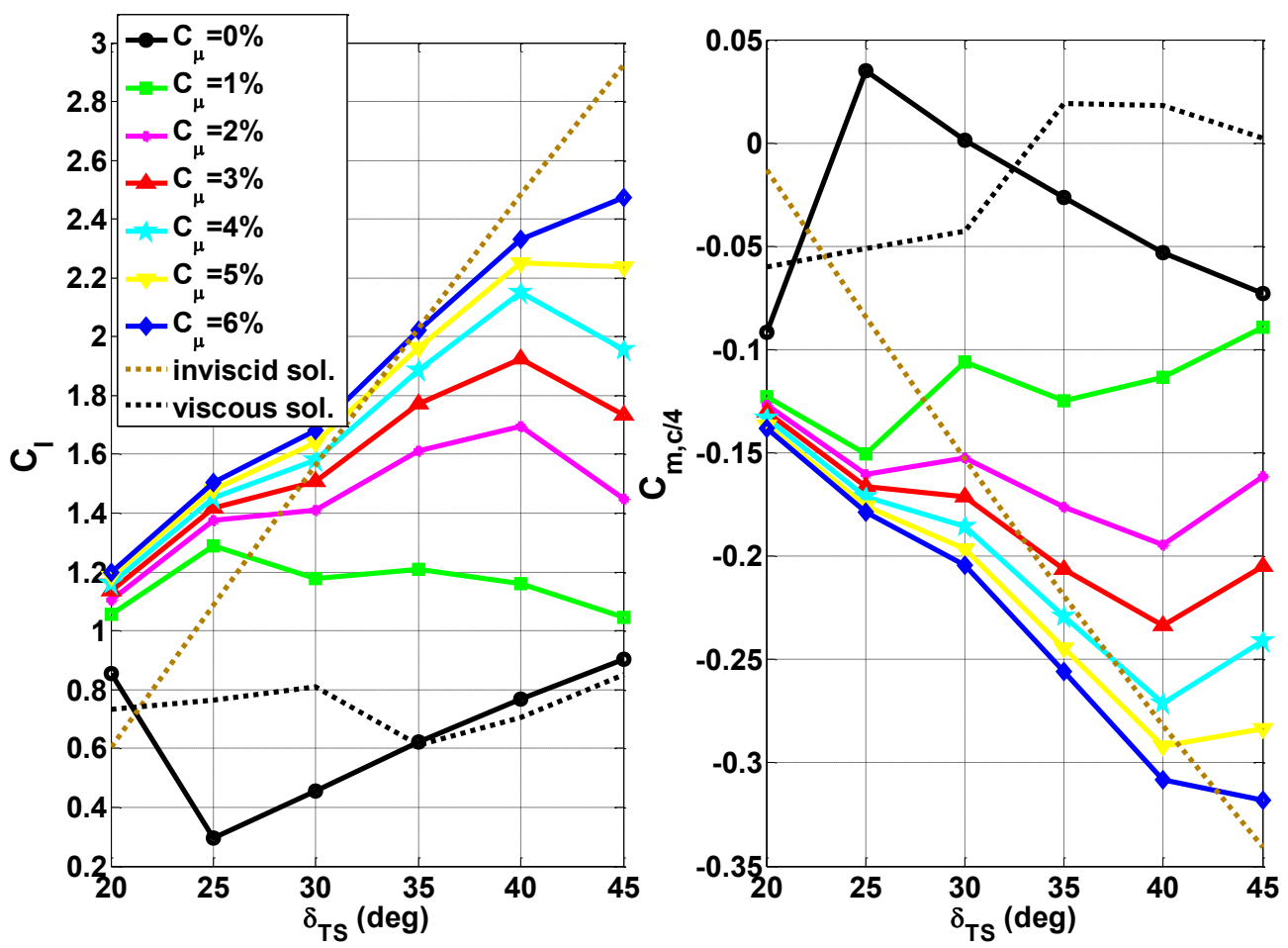

Figure 37 - Flap 40B, lift and moment vs. $\delta_{T S}, \alpha=0^{\circ}$, AFC @ 4\% $c_{f l a p}, \Delta z=1$ in.

For the flap configuration $40 \mathrm{~B}$ with actuation from $4 \% c_{\text {flap }}$, the lift and moment around the quarter chord are compared to the inviscid and viscous flow solution (Figure 37). As expected from potential flow theory, the lift and moment coefficient change linearly with flap deflection. The linear behavior is also evident for the experimental data 
for sufficiently high $C_{\mu}$. However, the slopes are different. For $\delta_{T S} \leq 30^{\circ}$, the lift obtained experimentally exceeds the simple potential flow solution that does not take jet entrainment into account. This may be interpreted at high input of momentum as creating supercirculation based on the jet-flap principle. At higher flap deflections more momentum is required to overcome separation and the remainder of the momentum input is insufficient to do so. Therefore, the experimental data falls below the predicted lift. The limitations of XFOIL are immediately noticeable when comparing the viscous baseline data in Figure 37. The predicted lift coefficients agree with the experiments as long as the flap deflection angles are well below or well above the threshold values for natural flow separation from the flap. The moment coefficient around the quarter chord point reveals even more pronounced deviations between calculations and experiments, suggesting that XFOIL does not predict separation well.

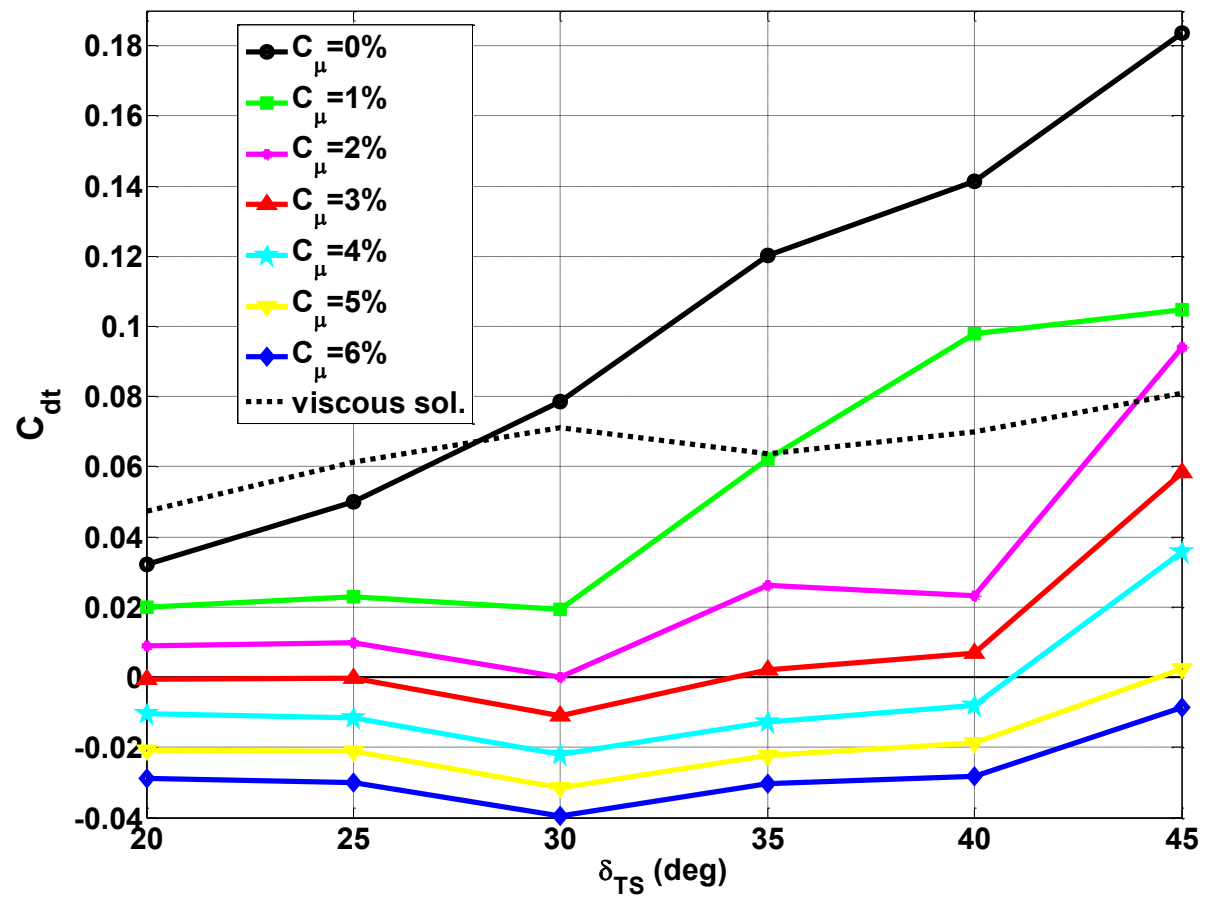

Figure 38 - Flap 40B, drag vs. $\delta_{T S}, \alpha=0^{\circ}$, AFC @ 4\% $c_{\text {flap }}, \Delta z=1$ in. 
Deviations between experiments and viscous predictions are also evident in the drag data (Figure 38). Although the agreement is close for small $\delta_{T S}$, XFOIL significantly underestimates the drag at higher $\delta_{T S}$. More interesting in Figure 38 is the total drag when applying actuation. As mentioned previously and indicated by the lift and moment data in Figure 37, the improvements are marginal once the flow over the flap is fully attached and any additional increase in $C_{\mu}$ is almost fully recovered in thrust. Since more momentum is necessary to attach the flow at larger $\delta_{T S}$, it would be expected that the total drag is increasing with $\delta_{T S}$. Instead, the drag is almost constant over the entire range of flap deflections with a minimum at $\delta_{T S}=30^{\circ}$. The explanation for this unexpected behavior is found in the particular geometry of the flap. In order to form the flap's straight upper surface, the bottom surface of the strongly converging trailing edge segment is deflected upwards by $34^{\circ}$ (see Figure 3 ). Therefore, the flow from this segment's bottom surface still separates for $\delta_{T S}=20^{\circ}$. Although actuation prevents separation from the flap's top surface, it is unable to control separation from the bottom surface. Later discussions of $C_{p}$-distributions provide further evidence for this behavior. The flow separation from the bottom surface adds to the total drag at small $\delta_{T S}$ and high $C_{\mu}$. As the flap deflection increases, the bottom surface of the trailing edge segment becomes more aligned with the free-stream and the flow remains attached thereby decreasing the total drag. However, now more momentum is necessary to control flow separation from the flap's top surface and consequently, less additional momentum is available to be recovered in thrust. This negates the drag reduction from attached flow at the bottom surface. A further increase in $\delta_{T S}$ maintains this balance until most of the 
momentum is required to control separation from the flap's top surface. A similar discussion is presented for a symmetric flap in the following chapter.

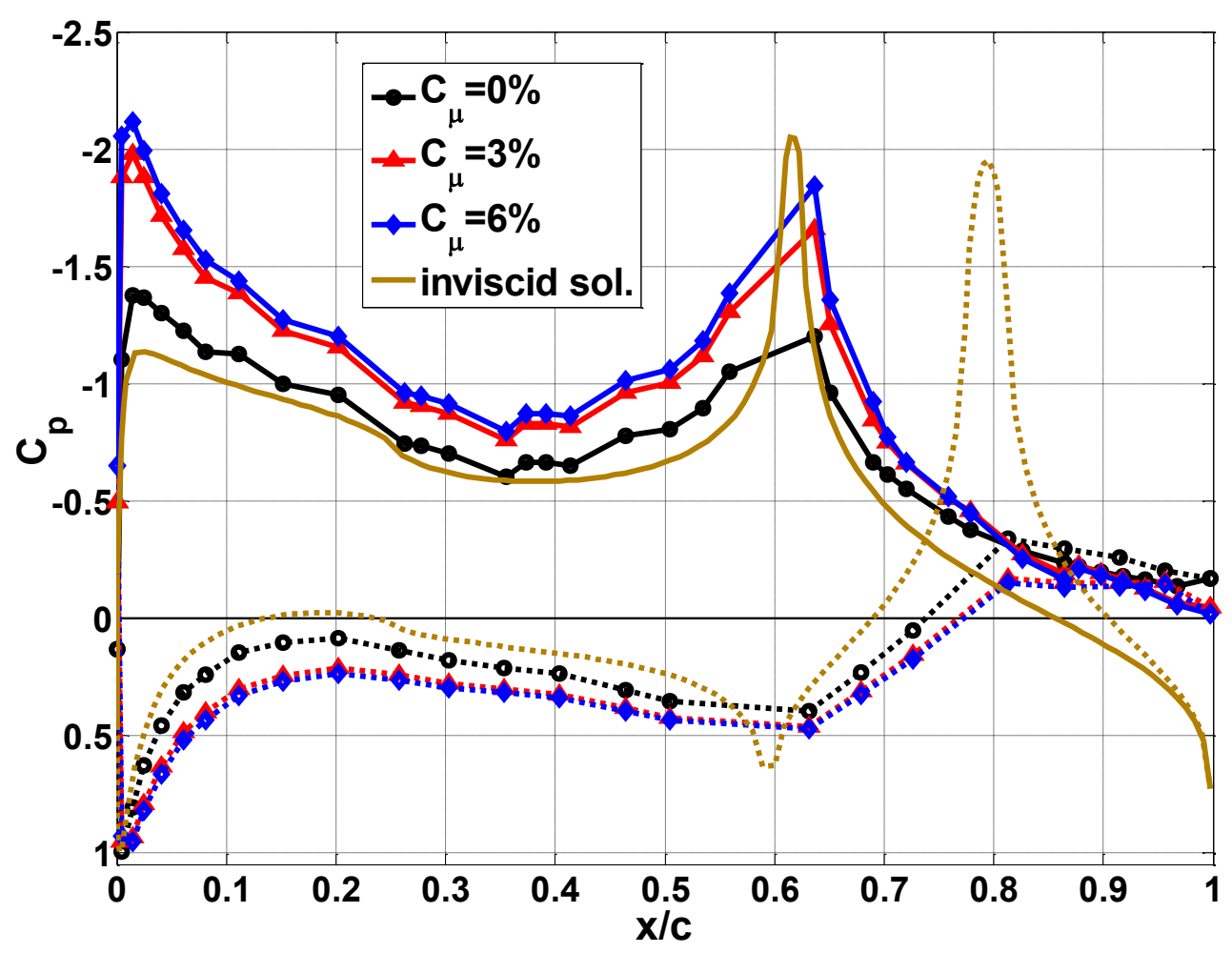

Figure 39 - Flap 40B, $C_{p}$-distributions, $\delta_{T S}=20^{\circ}, \alpha=0^{\circ}, \mathrm{AFC} @ 4 \% c_{\text {flap }}, \Delta z=1 \mathrm{in}$.

Although at first glance the computed values of lift and moment, that represent integral values, do not agree well with experiments (Figure 37), the evaluation of $C_{p^{-}}$ distributions (Figure 39-Figure 41) provides some explanations for these differences. With the top surface being flat, the bottom surface has a sudden $34^{\circ}$ divergence angle to the trailing edge (Figure 3). Therefore, the potential flow solution predicts a strong acceleration of the flow around the small but finite radius corner on the flap's bottom surface (Figure 39). The experimental data taken at $\delta_{T S}=20^{\circ}$ does not agree with the predicted acceleration at the flap's bottom surface because real fluid does not turn around sharp corners and it separates (e.g., Kutta condition on airfoils). It also does so on the top 
surface, despite the fact that it has to turn through only $20^{\circ}$. The experimental baseline case suggests that the flow separates downstream of the flap shoulder on the upper loft which is prevented by applying actuation from the top surface. However, even with strong actuation the pressures at the trailing edge and the bottom surface remain almost unchanged from the baseline pressure due to the thick boundary layer at the trailing edge. Thereby, the overall circulation is altered yielding a higher lift than provided by the inviscid solution. Although the total lift for $\delta_{f}<30^{\circ}$ is significantly larger than predicted by the inviscid solution, it is not due to supercirculation in the classical sense based on a jet-flap but rather due to the flap's specific geometry. If actuation were also applied at the flap bottom surface, better agreement with the inviscid solution may be achieved. With the additional control possibility at small $\delta_{T S}$, the strongly cambered flap design could be of interest to other applications that use similar flap geometries to control the aircraft's pitching moment (e.g., "Blended Wing Body").

As the flap is deflected to $30^{\circ}$ and $40^{\circ}$ (Figure 40 and Figure 41 ) the bottom surface of the trailing edge segment is almost aligned with the free-stream. Therefore, the acceleration of the flow around the $34^{\circ}$ corner at the flap's bottom surface is not as pronounced. Figure 40 (left) reveals a good agreement between the $C_{p}$-distribution of the AFC cases and the potential flow solution. At this combination of flap deflection and $C_{\mu}$ the pressure recovery over the flap's top and bottom surfaces matches the potential flow solution. Some of the discrepancies between the resulting lift and moment coefficients may partially be attributed to the lack of resolution in the experimental data. Since the velocities approaching the trailing edge from both surfaces of the airfoil have to be equal 
in order for the generated lift to be steady, actuation from the flap's top surface also affects the pressure distribution on the lower surface. It should be noted that the difference between $C_{\mu}=3 \%$ and $C_{\mu}=6 \%$ is only evident upstream of the actuation location because the lower $C_{\mu}$ does not manage to turn the flow as sharply as it may be needed. The question arises whether a further increase in $C_{\mu}$ may yield a considerably larger lift coefficient than predicted by the inviscid calculation. Since the flow is fully attached over the entire model, this kind of supercirculation should originate from the classical concept of a strong jet departing from the trailing edge and thereby increasing the circulation around the model. Figure 40 (right) illustrates the measured wake profiles corresponding to $\delta_{T S}=30^{\circ}$. For $C_{\mu}$ values of $3 \%$ and $6 \%$, the velocity profile downstream of the model changes from the broad wake profile corresponding to the baseline configuration to a jetlike profile, thereby providing net thrust. The wake's center is shifted downward inferring that lift is generated. Based on the jet-flap concept one may expect a lift coefficient that exceeds the potential flow prediction. However, the jet departing the trailing edge may not be strong enough or may not have enough downward momentum. One may also note that the recorded pressures in the wake represent an average that does not account for possible oscillatory changes along the span. These oscillations may prevent the formation of a continuous "jet curtain" and the establishment of a pressure discontinuity across the jet sheet as it is the case in the classical concept of a jet-flap. Only unreasonably higher values of $C_{\mu}$ may be capable of achieving to achieve supercirculation in the classical sense if indeed the spanwise periodic sweeping jets are capable of forming a continuous jet sheet along the span. 

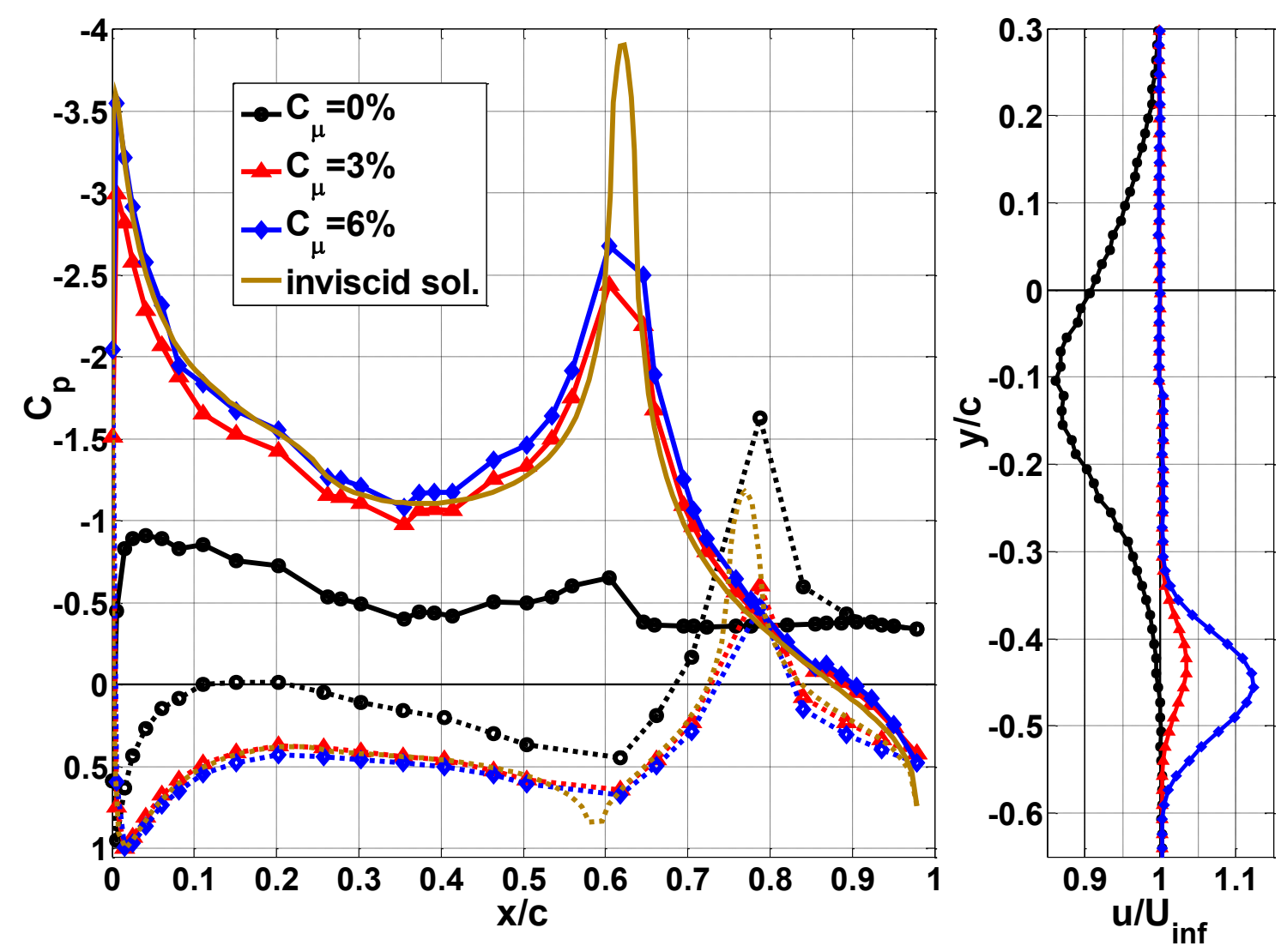

Figure 40 - Flap 40B, $C_{p}$-distributions $\&$ wake profiles, $\delta_{T S}=30^{\circ}, \alpha=0^{\circ}, \mathrm{AFC} @ 4 \% c_{f l a p}, \Delta z=1$ in.

If $\delta_{T S}$ is increased to $40^{\circ}$ (Figure 41 ), the momentum input is not sufficient to obtain the lift predicted by inviscid theory. Although the flow is fully attached over the flap, yielding the predicted pressure recovery at the trailing edge, the required acceleration near the leading edge did not materialize resulting in too high a pressure over the main element's top surface. Even though a $C_{\mu}$ of $3 \%$ suffices to attach the flow downstream, $C_{\mu}=6 \%$ accelerates the flow somewhat better upstream of the actuation location. 


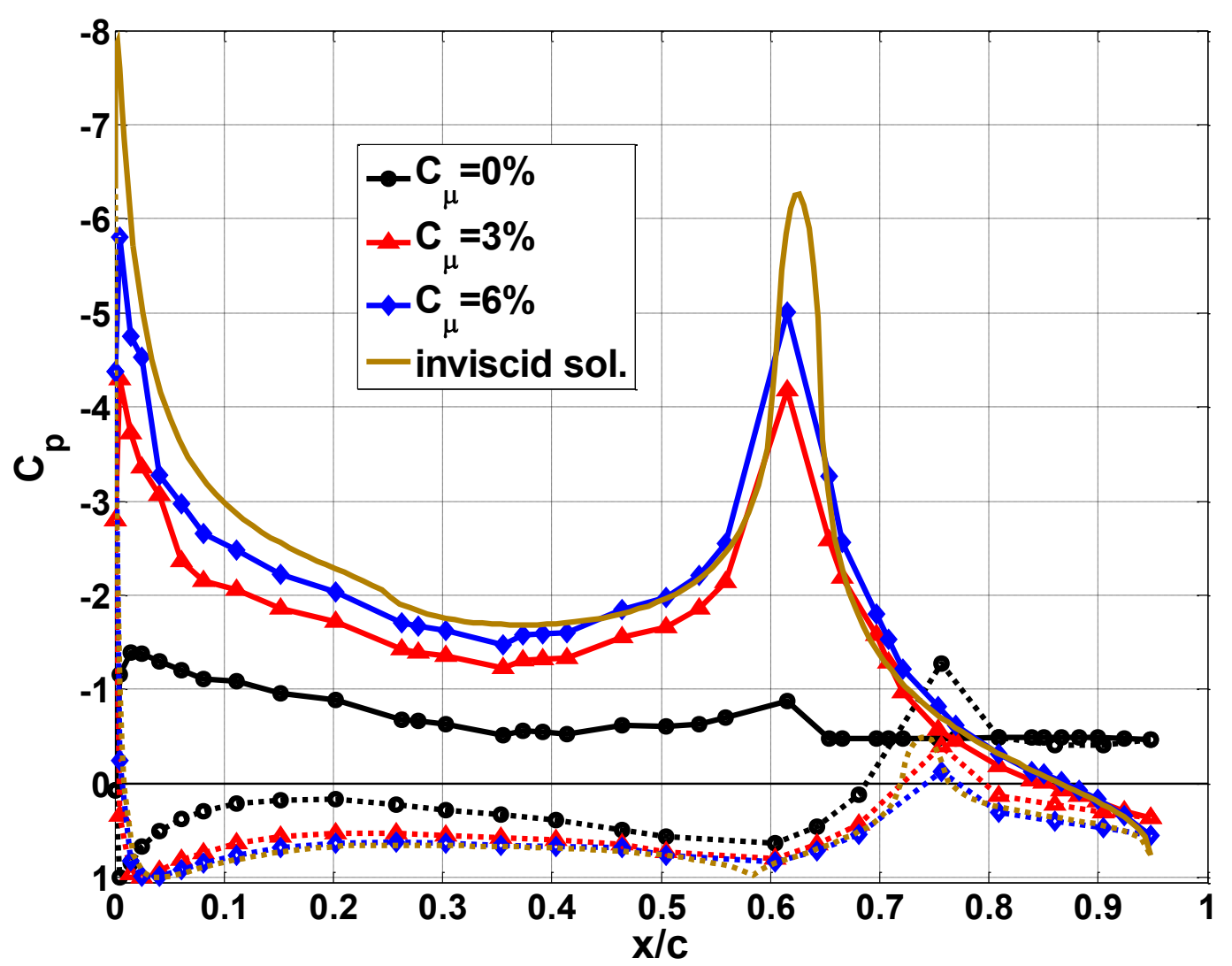

Figure 41 - Flap 40B, $C_{p}$-distributions, $\delta_{T S}=40^{\circ}, \alpha=0^{\circ}$, AFC @ $4 \% c_{\text {flap }}, \Delta z=1$ in.

\subsubsection{Symmetric Flap}

Due to the strong effect of the flap's inflected camber-line, the configuration 40B may not be an appropriate example to verify the claim that an inviscid solution is a valuable predictor for the lift generated with sweeping jets at reasonable values of $C_{\mu}$. Therefore, the same exercise as discussed in the previous section is repeated with the flap configuration $40 \mathrm{~A}$ which has the same size flap but with a symmetric geometry.

Due to the reduced effect of the flap's bottom surface, the agreement between the inviscid flow solution and the experimental data is significantly improved over a wider range of flap deflections (Figure 42). The lift and moment change linearly with $\delta_{T S}$ at 
almost the same slope. However, observations similar to the cambered flap are noted. At small $\delta_{T S}$ the lift achieved experimentally exceeds the potential flow predictions, whereas for high $\delta_{T S}$ the situation is vice versa. The reasons for these deviations are analogous to the cambered flap and discussed shortly in conjunction with pressure distributions. Due to the absence of the low pressure region at the flap's bottom surface, the symmetric flap yields a maximum lift coefficient of 2.9 instead of 2.5. The nose-down pitching moment is also significantly higher than for the cambered flap. Note that an increase in $C_{\mu}$ only yields marginal changes once the flow is fully attached. These limiting values of lift and moment agree well with the inviscid solution supporting the argument that efficiently achievable improvements with sweeping jets can be estimated by potential flow theory.
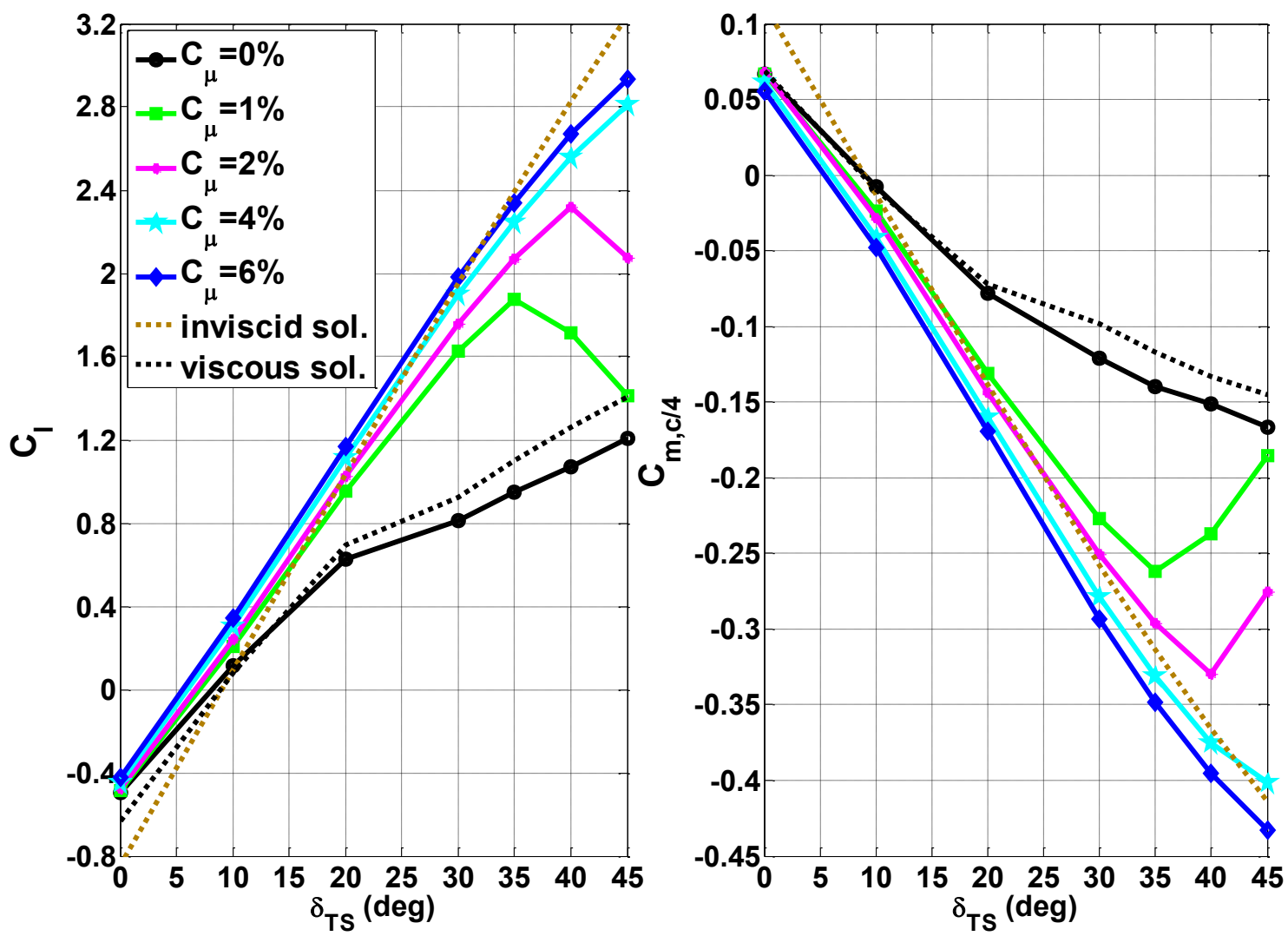

Figure 42 - Flap 40A, lift and moment vs. $\delta_{T S}, \alpha=0^{\circ}$, AFC @ 7\% $c_{f l a p}, \Delta z=1 \mathrm{in}$. 
The simpler geometry of the flap also improves the viscous solution obtained with XFOIL (Figure 42 and Figure 43). Especially for smaller flap deflections the agreement with the experimental baseline data is satisfactory. However, with the presence of strongly separated flow, XFOIL underestimates the effects of separation, again revealing that XFOIL is not suitable for these flow scenarios.

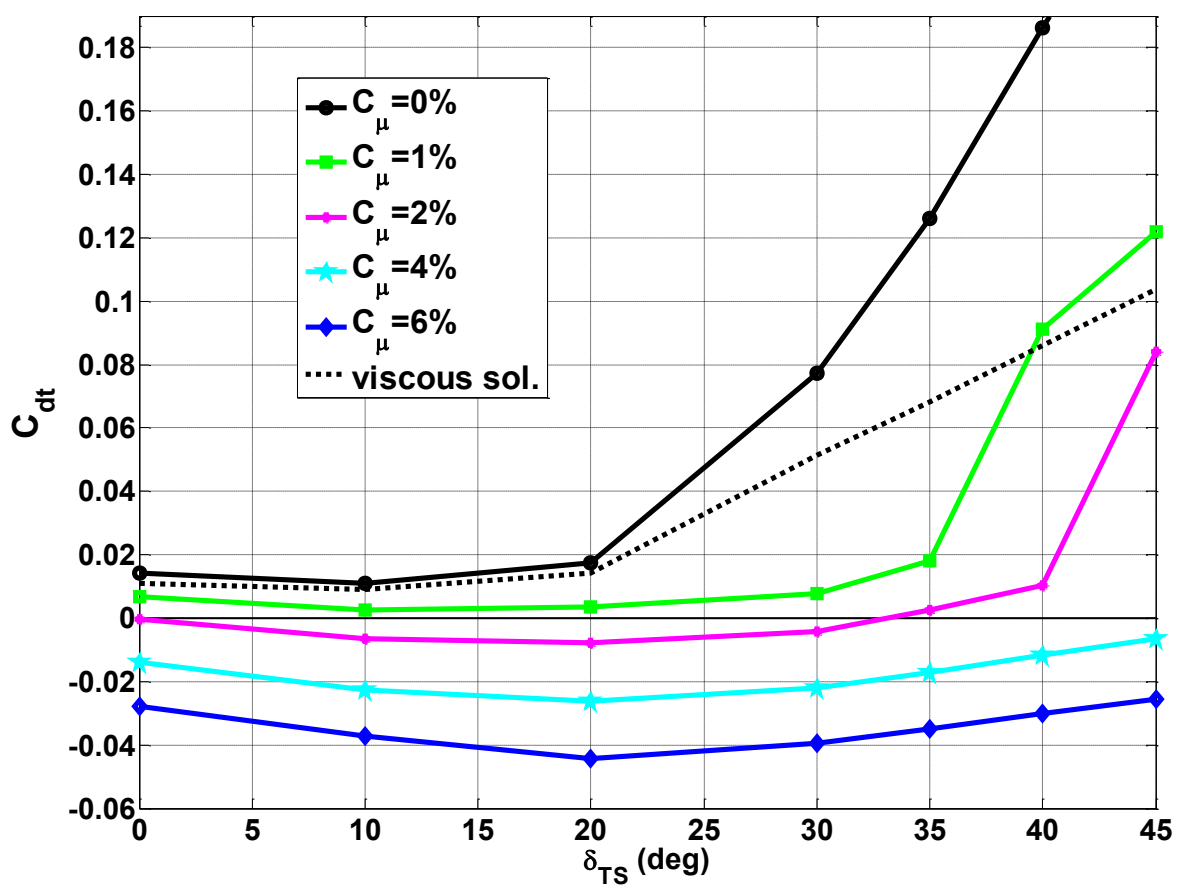

Figure 43 - Flap 40A, drag vs. $\delta_{T S}, \alpha=0^{\circ}, \mathrm{AFC} @ 7 \% c_{\text {flap }}, \Delta z=1$ in.

Figure 43 also reveals interesting trends for the total drag when actuation is applied. Again, the drag does not consistently increase with flap deflection, indicating a minimum at $\delta_{T S}=20^{\circ}$. At $\delta_{T S}=0^{\circ}$ the flap's bottom surface is deflected upwards by $18^{\circ}$, which causes a thick boundary layer at the flap's bottom surface and adds to the total drag. The injection of momentum from the top surface has no effect on the flow over the bottom surface (Figure 44). If $\delta_{T S}$ is increased to $20^{\circ}$ the flap's and main element's lower lofts are aligned, thereby reducing the momentum deficit caused by the flow over the 
flap's bottom surface. Actuation at small levels is sufficient to control separation from the flap's top surface. Therefore, any additional momentum is recovered in thrust yielding a minimum in the total drag. For larger flap deflections more $C_{\mu}$ is required to control separation leaving less additional momentum for thrust recovery. Therefore, the total drag is consistently increasing with flap deflection for $\delta_{T S} \geq 20^{\circ}$. Note that, once the flow is fully attached, the additional momentum is almost fully recovered in thrust supporting the $C_{\mu}$ calculations even for $\Delta z=1$ in. which inherits higher jet velocities than $\Delta z=0.5 \mathrm{in}$.

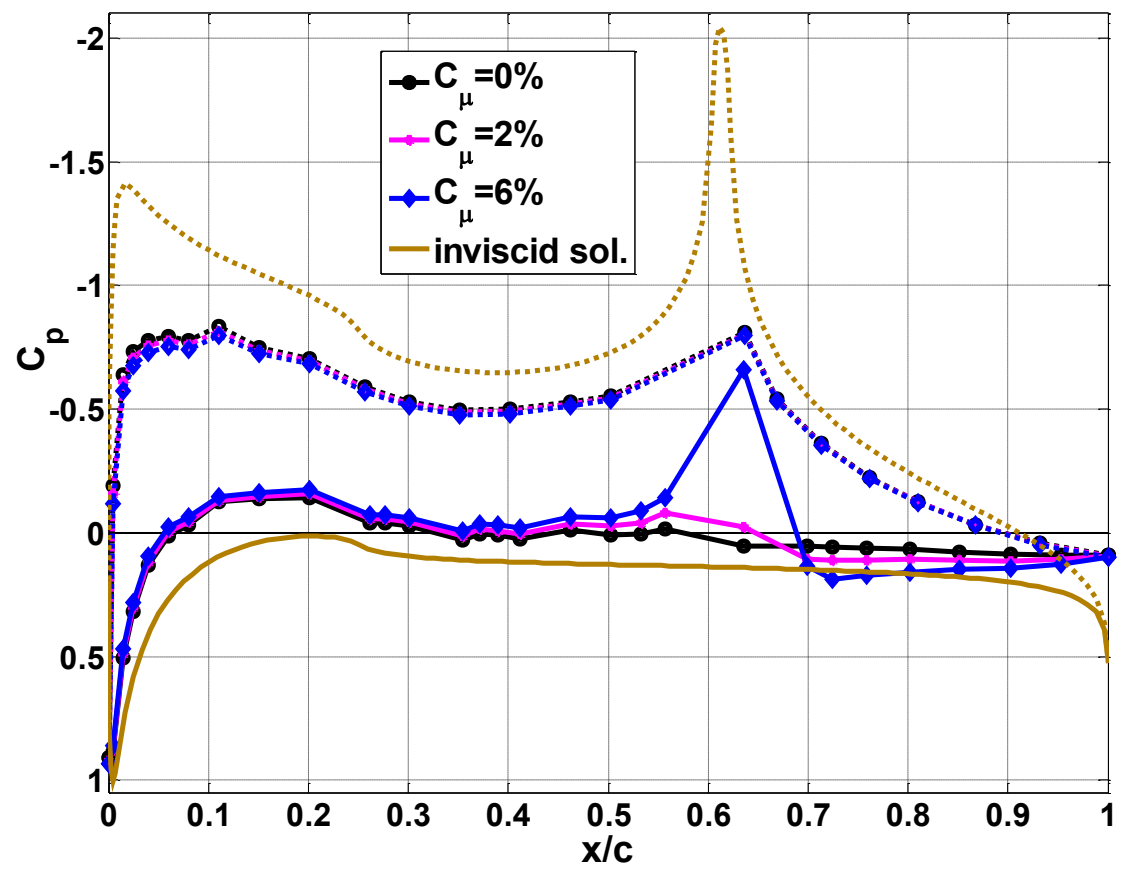

Figure 44 - Flap 40A, $C_{p}$-distributions, $\delta_{T S}=0^{\circ}, \alpha=0^{\circ}, \mathrm{AFC} @ 7 \% c_{f l a p}, \Delta z=1$ in.

Further explanations for the aforementioned observations are provided by pressure distributions for selected cases (Figure 44-Figure 46). Figure 44 illustrates the $C_{p}$-distribution for $\delta_{T S}=0^{\circ}$. The dotted lines represent the pressure distribution along the airfoil's bottom surface. Due to the upward deflection of the flap's bottom surface, the inviscid flow solution predicts a strong acceleration of the flow around the $18^{\circ}$ turn. The 
prediction does not agree with the experimental data because the viscous flow inherits at least a thick boundary layer, which reduces the flow acceleration over the main element's bottom surface. These effects are also evident in the pressure recovery at the trailing edge. Even with actuation the experimental pressure recovery is smaller than the inviscid prediction. Actuation from the flap's top surface does not affect the flow at the model's bottom surface and only yields localized acceleration upstream of the actuation location. Although actuation is applied at high $C_{\mu}$, the effect on the overall circulation is minor. This supports the argument that sweeping jets are not just effective in controlling flow separation due to the momentum they insert, but mainly due to the effective structures they form (i.e., streamwise vortices).

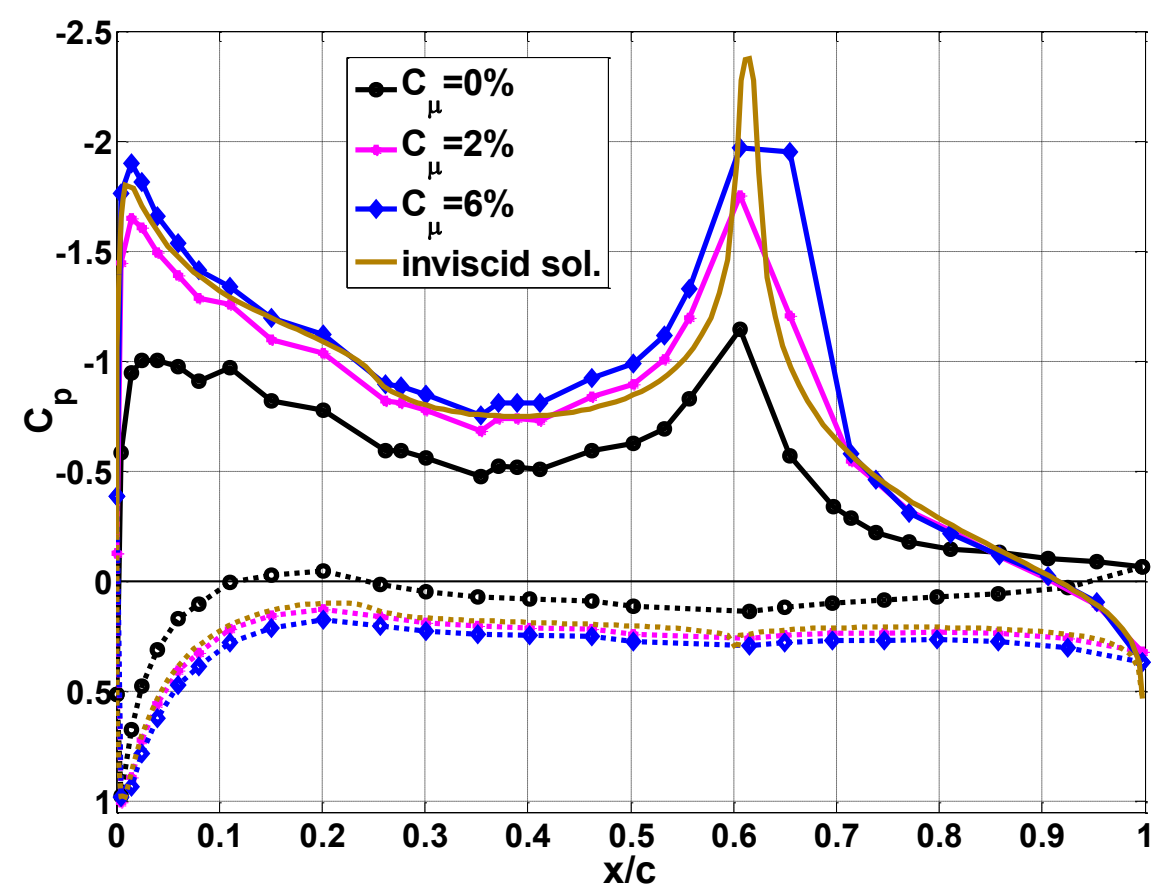

Figure 45 - Flap 40A, $C_{p}$-distributions, $\delta_{T S}=20^{\circ}, \alpha=0^{\circ}, \mathrm{AFC} @ 7 \% c_{\text {flap }}, \Delta z=1$ in.

With an increased flap deflection $\left(\delta_{T S}=20^{\circ}\right)$ the flap's and main element's bottom surface are aligned, which significantly improves the agreement between experimental 
and numerical data when controlling flow separation over the flap's top surface (Figure 45). The pressure recovery at the trailing edge agrees well with the inviscid prediction. Additional input of momentum causes increased flow acceleration at and upstream of the actuation location which adds some minor improvements in lift beyond the potential flow solution.

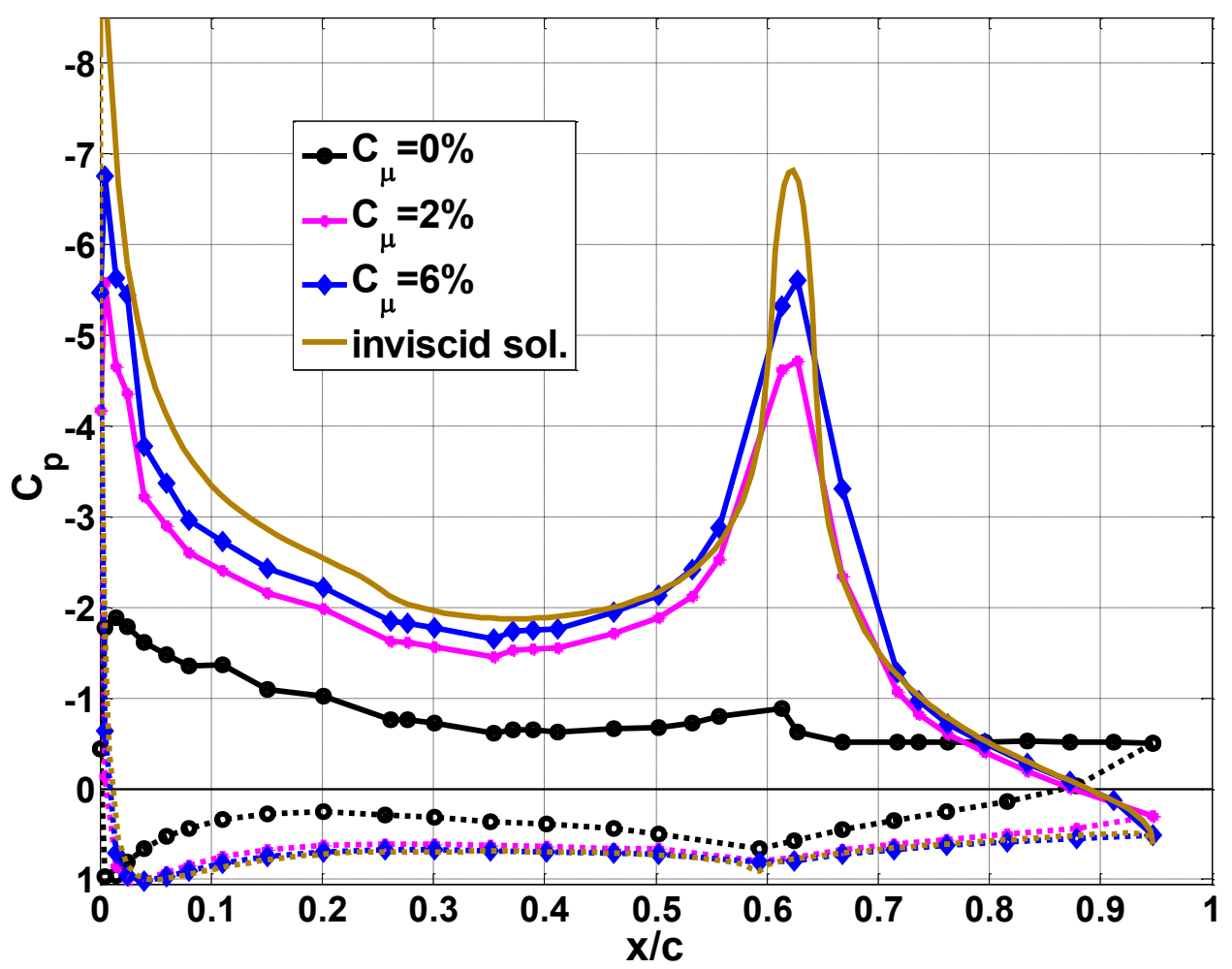

Figure 46 - Flap 40A, $C_{p}$-distributions, $\delta_{T S}=40^{\circ}, \alpha=0^{\circ}$, AFC @ 7\% $c_{\text {flap }}, \Delta z=1$ in.

Similar to the cambered flap, the limitations of sweeping jets become evident for higher flap deflections (Figure 46). Although the flow is fully attached over the flap, yielding the predicted pressure over the flap and the airfoil's bottom surface, the flow is not turned sharply enough around the $40^{\circ}$ divergence at the flap shoulder. Therefore, sweeping jets are not able to achieve the predicted flow acceleration over the main element's top surface which results in a smaller lift. 


\subsection{Effects of Flap Deflection}
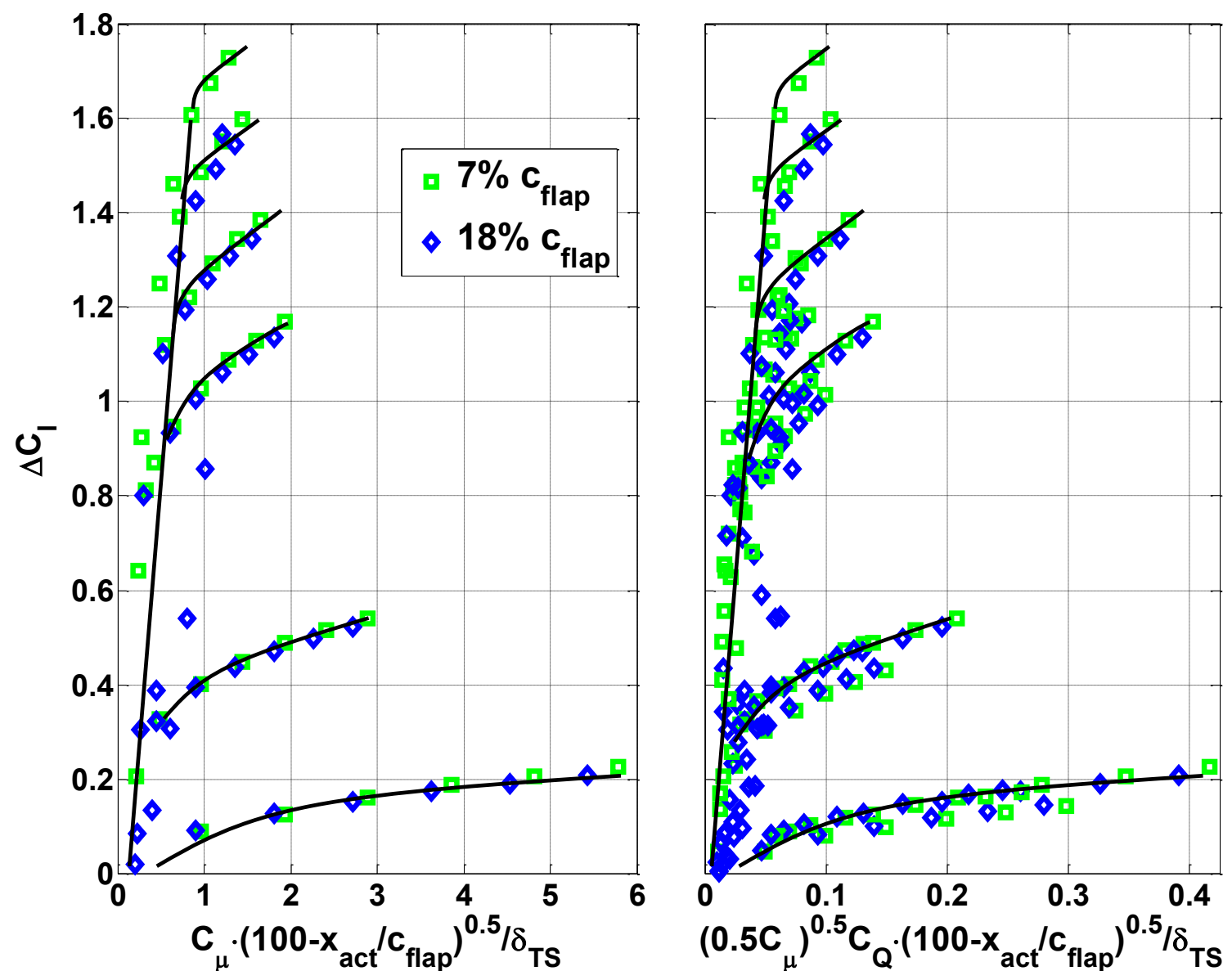

Figure 47 - Flap $40 \mathrm{~A}, \Delta C_{l}$ vs. $C_{\mu} \cdot\left(100-x_{a c} / c_{f l a p}\right)^{0.5} / \delta_{T S}($ left, $\Delta z=1 \mathrm{in}). \&\left(0.5 C_{\mu}\right)^{0.5} \cdot C_{Q} \cdot\left(100-x_{a c} / c_{f l a p}\right)^{0.5} / \delta_{T S}$ (right, all $\Delta z$ ), $\alpha=0^{\circ}$.

The repeated reference to flap deflection in previous chapters almost exhausts the discussion of this parameter. It is indicated at multiple occasions that the momentum input required to attach the flow increases as the flap is further deflected and actuation applied further downstream on the flap. This statement is summarized in Figure 47 (left) by a single coefficient which combines the effects of actuation intensity, location, and flap deflection by extending the coefficient proposed in Figure 35 with the flap angle. Actuation from the flap shoulder is omitted due to the aforementioned discrepancies with 
this location. The differential lift increases almost linearly with the coefficient $C_{\mu}(100-$ $\left.x_{a c t} / c_{f l a p}\right)^{0.5} / \delta_{T S}$ for all actuation locations and flap deflections. However, curves branch away from the linear pattern at similar slopes visualizing the limitations in $\Delta C_{l}$ once the flow over the flap is fully attached and $C_{\mu}$ continues to increase.

Because the coefficient in Figure 47 (left) is only considered for a fixed actuator spacing, it may be extended by the coefficient proposed in Figure 14. The data for all three spacings shown in Figure 47 (right) resemble the same trends as for a single spacing thereby providing one single coefficient which exposes the relationship between actuation intensity (i.e., $C_{\mu}$ and $C_{Q}$ ), actuation location, and flap deflection. The applicability of this coefficient was verified for other flap sizes and geometries yielding the same trends.

\subsection{Effects of Flap Size}

In addition to the flap deflection angle, the geometric potential to provide a desired lift is also dependent on the flap size. Although some references to this parameter are discussed in preceding chapters (e.g., Chapter 5.1), the following arguments offer additional information pertaining to flap size. The data are focused on $\alpha=0^{\circ}$ as the effects of incidence are addressed in the subsequent chapter.

One of the main effects noticed for various flap sizes is the difference in momentum input required to attach the flow over the flap. Figure 48 (left) illustrates the relative lift improvements for all flaps deflected at $\delta_{T S}=40^{\circ}$ as a function of $C_{\mu}$. It is evident that with increasing flap size more momentum is necessary to attach the flow over the flap. Although actuation has to turn the flow around the same angle for all flaps, 
it has to do so over a longer downstream distance. Therefore, $C_{\mu}$ increases with flap size. Due to different baseline values, different levels of relative improvements are achieved. When considering the momentum coefficient based on the flap chord (i.e., $C_{\mu, f l a p}$ ), the agreement between all flap sizes is significantly improved (Figure 48, right) implying that the momentum input is more suitably scaled by the length of the flap instead of the total chord length. This statement may even be generalized by suggesting that the momentum input scales with the size of the separated area to be controlled. Once the flow over the flap is attached, the curves in Figure 48 diverge which originates from the different baseline characteristics and the presentation of relative lift improvements.

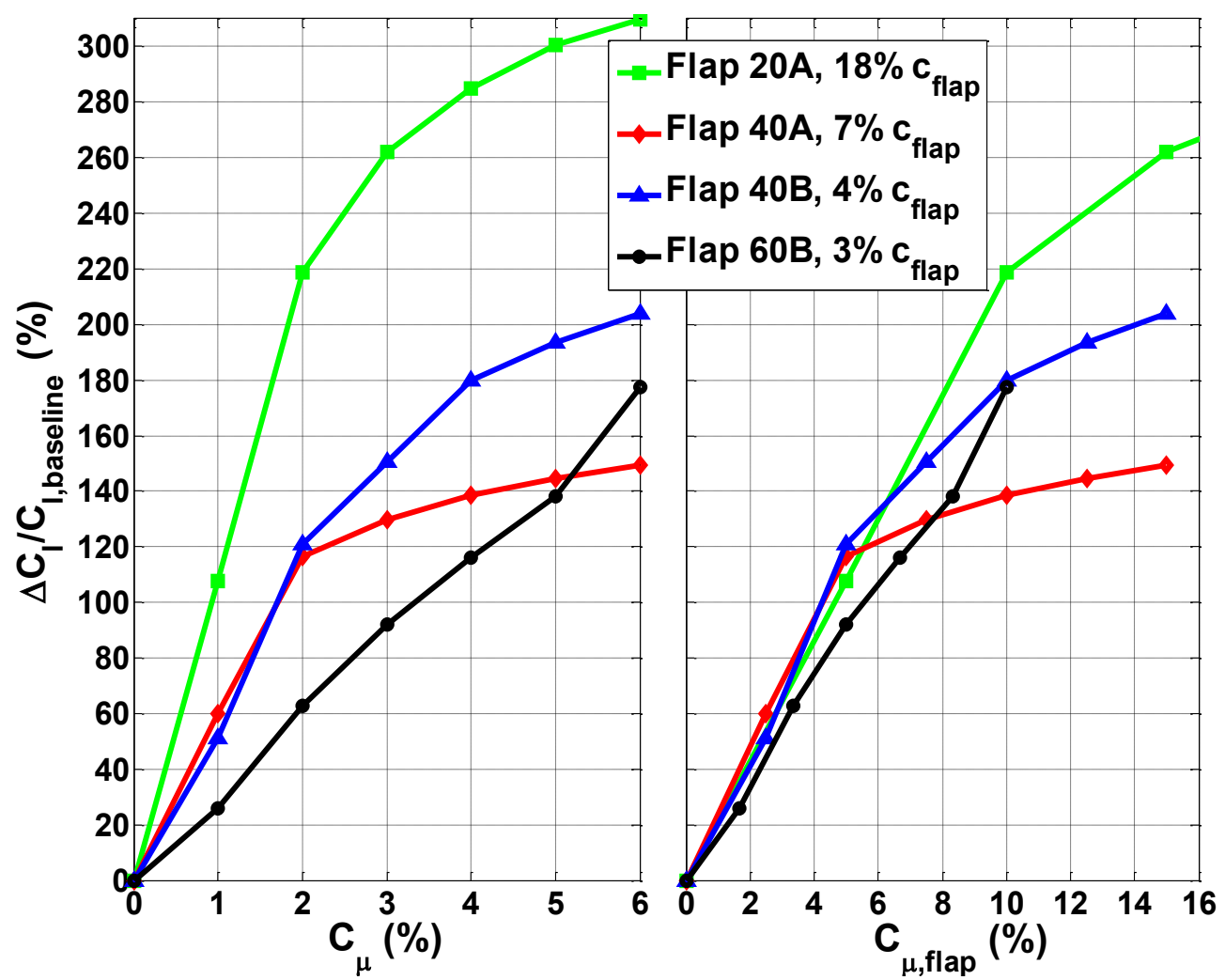

Figure 48 - All flaps, differential lift improvements vs. $C_{\mu} \& C_{\mu f l a p}, \delta_{T S}=40^{\circ}, \Delta z=1$ in. 
The good agreement between various flap sizes also supports the reference of flap deflection to the flap's top surface (i.e., $\delta_{T S}$ ) and not to the flap's chord line (i.e., $\delta_{f}$ ). Although the flow from the flap's lower loft affects the upper loft as well, the main task of separation control is to overcome the divergence angle between the main element and the flap's upper surface. Therefore, the common notation originating from thin airfoil theory may not be appropriate for considerations of separation control especially for thick airfoils and short flaps with a steep convergence to the trailing edge.

Although a larger flap size requires a higher momentum input to attach the flow, it also yields a larger lift due its greater geometric potential. A prescribed lift may be achieved at smaller flap deflections which require a smaller momentum input. Therefore, the choice of flap size will depend on which flap can yield a desired lift most efficiently. In order to address this question, the various flap sizes are evaluated based on the smallest corrected drag for a prescribed lift. The drag corrections are either based on $C_{\mu}$ (Equation (9)) or on $C_{E}$ (Equation (10)). For a desired lift value, $C_{l, d e s}$, the configuration with the smallest corrected drag is found from the entire range of flap deflections and momentum coefficients. Due to the inherent differences in flap geometry, the discussion focuses first on the flaps $60 \mathrm{~B}$ and $40 \mathrm{~B}$ (Figure 49) and then on the flaps $40 \mathrm{~A}$ and $20 \mathrm{~A}$ (Figure 49).

As expected, the configuration 60B yields a larger maximum lift and requires smaller flap deflections with smaller momentum input than the $40 \%$ flap. However, the larger flap is penalized with a larger drag over the entire range of lift values as reflected by $\eta_{C_{\mu}}$. Only when approaching $C_{l, \max }$, the performances become comparable. Note that 
discontinuities in $C_{\mu}$ and $\eta_{C_{\mu}}$ are consistent with changes in flap deflection. In order to obtain a certain lift at a certain flap angle, the momentum input increases until the efficient usage of the geometric potential is exhausted and the process starts over at a higher $\delta_{T S}$. The variations in $\eta_{C_{\mu}}$ are also influenced by an amplifying sensitivity to small changes in drag as the lift values increase. Even though one may argue that the flap geometries are too different for this comparison to hold any merit, it should be noted that the inflected trailing edge flap segment entails half the size of the $40 \%$ flap whereas it is only a third of the $60 \%$ flap. Therefore, the differences between a $60 \%$ and $40 \%$ flap may even be more pronounced in absence of this segment's lift reducing effect.
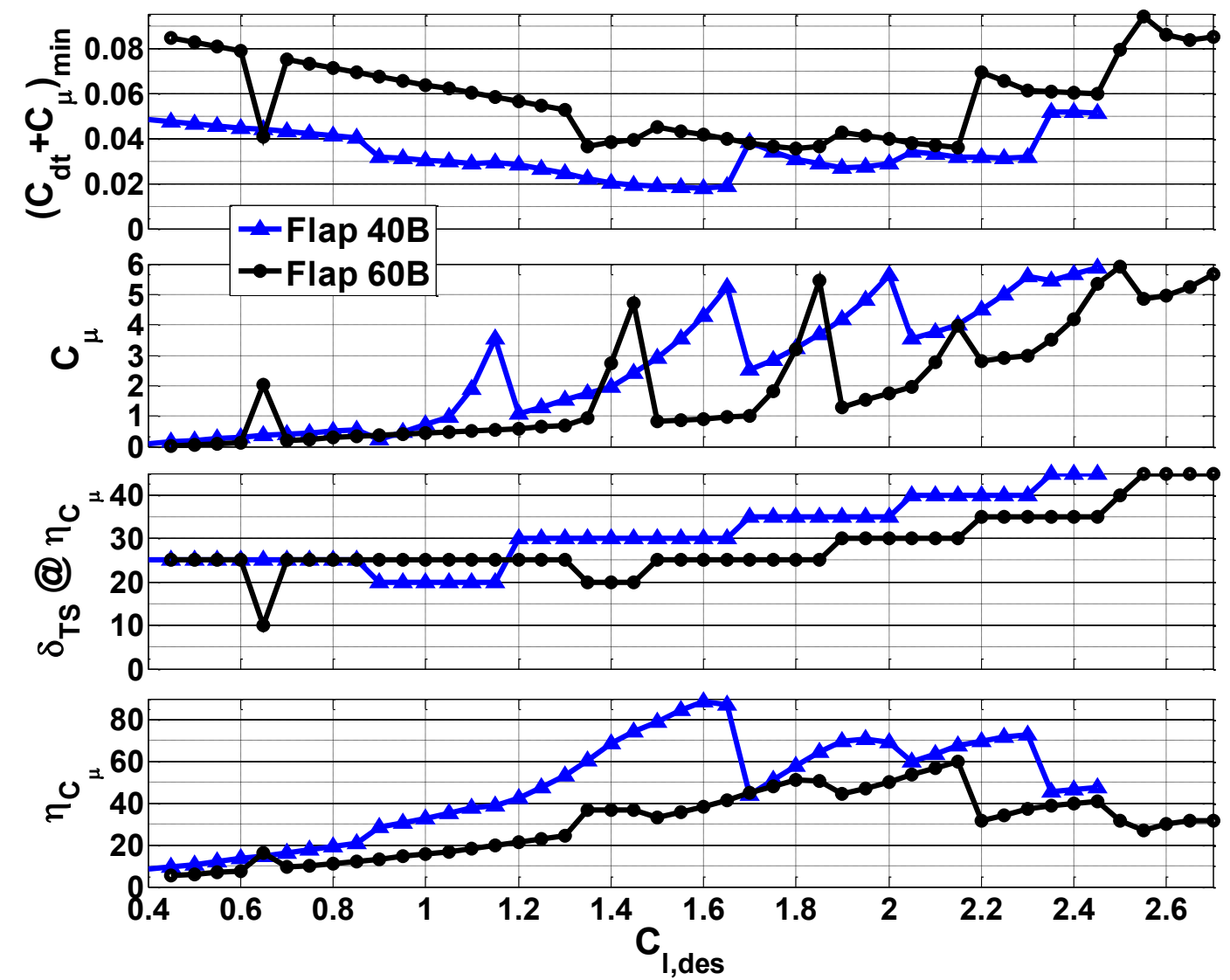

Figure 49 - Flap 60B \& 40B, best performance based on $\left(C_{d t}+C_{\mu}\right)_{\min }$ vs. $C_{l, d e s}$, best location, $\Delta z=1 \mathrm{in}$. 
Similar to the discussion of Figure 49, the comparison is repeated for the flap configurations 40A and 20A (Figure 50). Due to the small flap and the steep convergence to the trailing edge, the $20 \%$ flap yields a significantly smaller range of achievable lift values. The small flap requires a larger $C_{\mu}$ and $\delta_{T S}$ to achieve the same lift as the $40 \%$ flap. Only for a small range of $C_{l, d e s}$ does the $20 \%$ flap perform better than the larger flap. Nevertheless, both flap sizes are able to maintain a high performance level over a wide range, but these levels are higher for the $40 \%$ flap and the respective range of $C_{l, d e s}$ is shifted to larger values. Due to the inherent differences in flap geometry, the comparison between the configurations $40 \mathrm{~A}$ and $20 \mathrm{~A}$ is not as conclusive as for $60 \mathrm{~B}$ and $40 \mathrm{~B}$. However, similar trends are observed.

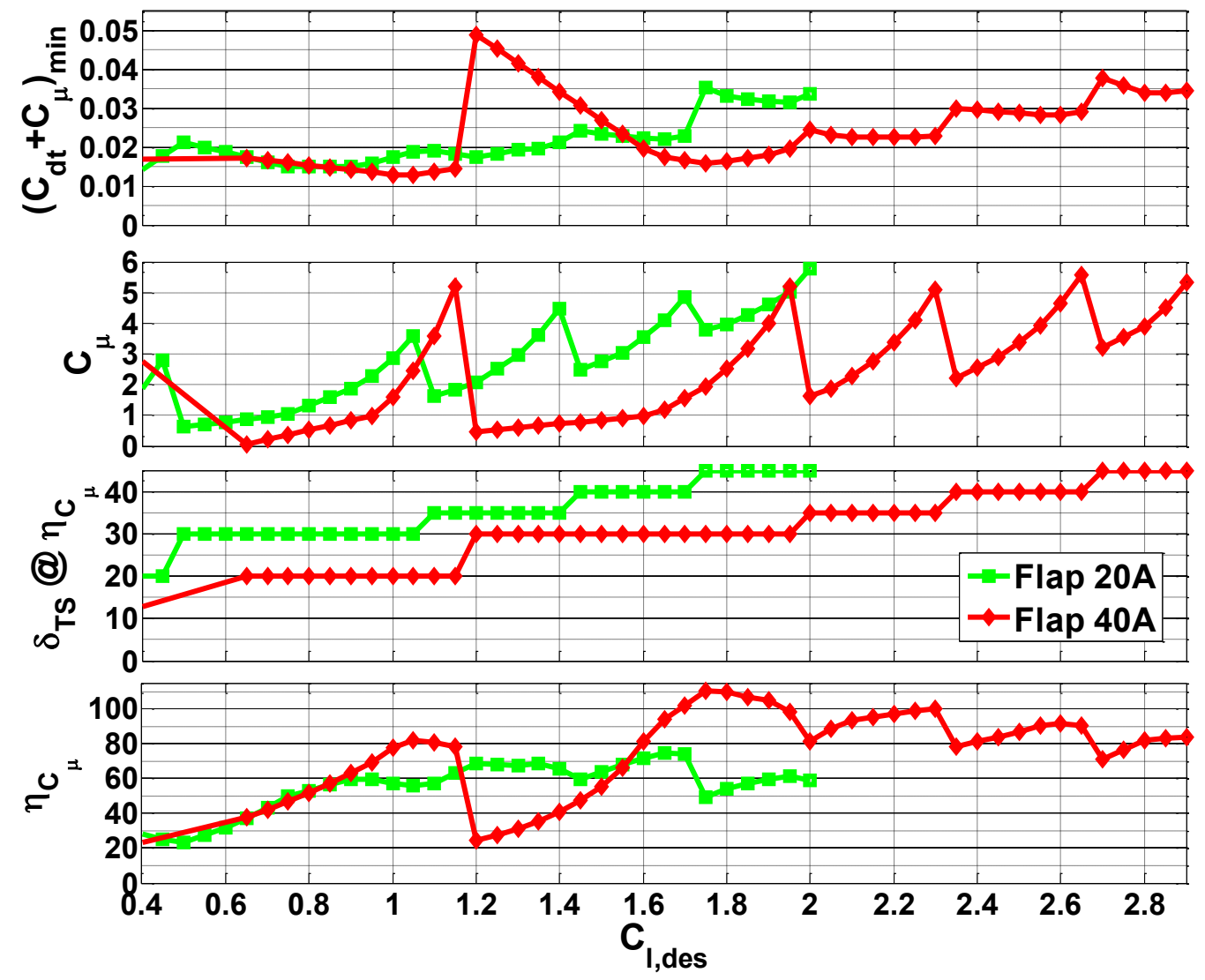

Figure 50 - Flap $40 \mathrm{~A} \& 20 \mathrm{~A}$, best performance based on $\left(C_{d t}+C_{\mu}\right)_{\min }$ vs. $C_{l, d e s}$, best location, $\Delta z=1 \mathrm{in}$. 
The comparison between the different flap sizes may be repeated for drag corrections based on $C_{E}$. However, since the general trends are maintained, these figures are omitted.

Based on this large data set at $\alpha=0^{\circ}$, including flap size, flap deflection, momentum input, and actuator spacing, an attempt is made to summarize the improvements obtained from controlling separation with sweeping jets. Figure 51 displays the maximum performance values (i.e., $C_{l, \max }, \eta_{\max }, F M_{\max }$, and $C_{l} @ \eta_{\max }$ ) based on $C_{\mu}$ and $C_{E}$ as a function of actuator spacing. As argued before, the highest lift is achieved with sweeping jets emitted at $\Delta z=1$ in. The maximum lift and all other coefficients also reiterate the fact that the maximum spacing between adjacent actuators is limited. A spacing of $\Delta z=1.5 \mathrm{in}$. is significantly less effective and efficient than the smaller spacings. Figure 51 also indicates that for achieving a maximum corrected lift-todrag ratio (i.e., $\eta_{\max }$ ) an optimum flap size has to be found which balances the size of the separation region with the geometric potential to produce lift. If the flap is too small, its ability to provide lift is limited. If the flap is too large, the increased lift is penalized by more drag. This relationship is also resembled by the maximum figure of merit which provides a measure for the relative improvements when compared to the maximum baseline performance (Equation (12)). The most impressive performance increase is achieved for the simple $40 \%$ flap. Even when correcting the drag with $C_{E}$, actuation increases the maximum $L / D$ by a factor of 15 . If the total drag is corrected by $C_{E}$ instead of $C_{\mu}$, generally the same trends are observed. However, the differences between the various flap sizes are less pronounced. This illustrates that the measure of performance is 
dependent on the respective criteria. Although the smallest spacing appears most efficient due to the smaller $C_{\mu}$ values, a spacing of $\Delta z=1$ in. may be more optimal if the highest $C_{l, \max }$ and smallest flowrate are desired (e.g., for a vertical tail).
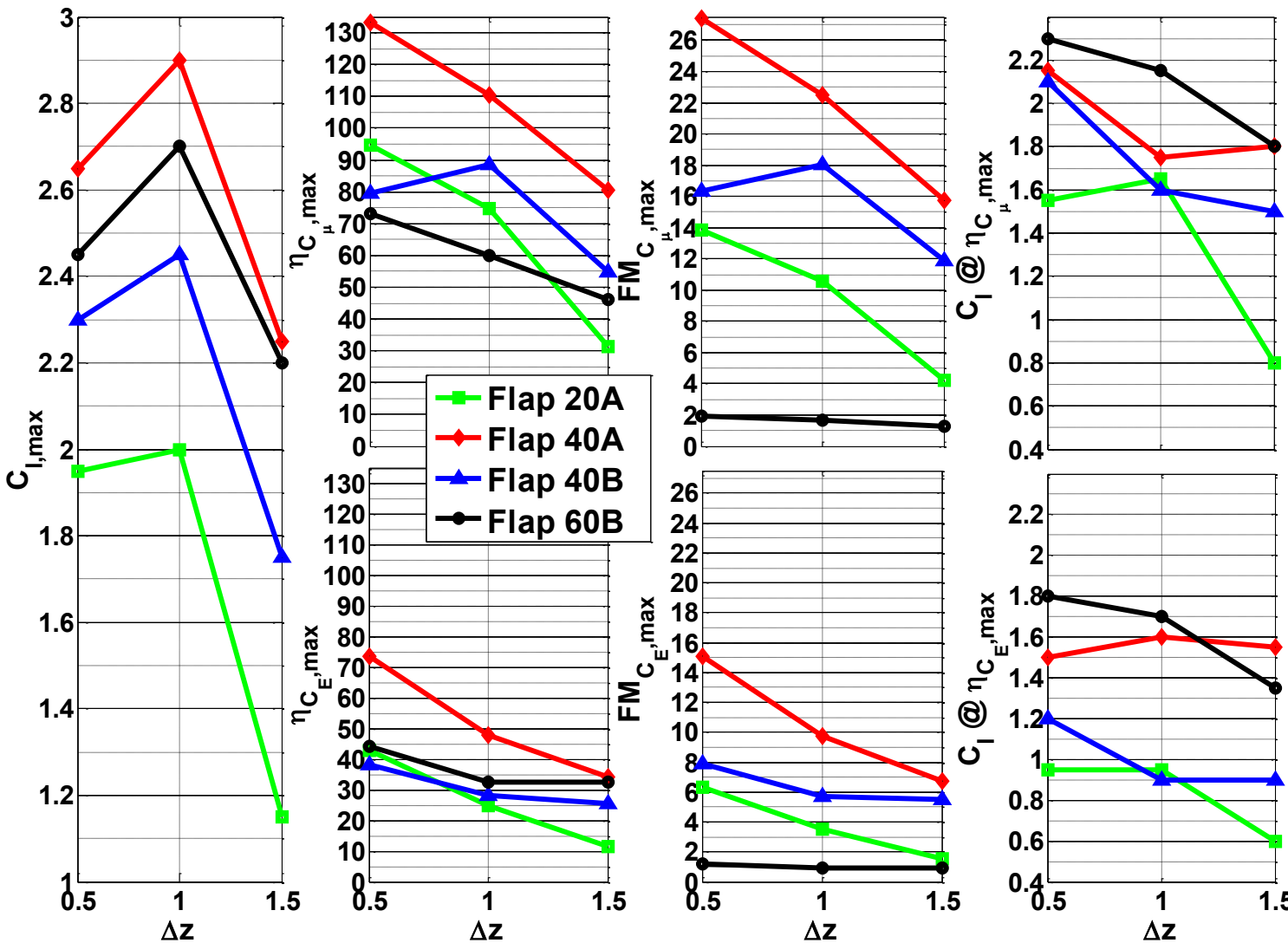

Figure 51 - All flaps, maximum performance coefficients vs. $\Delta z, \alpha=0^{\circ}$, best actuation locations.

Although the discussion of flap size reveals some dominant trends and valuable information, it is left with some uncertainty due to the inherent differences in flap geometry. Future experiments, involving a small and large model with the same percentage flap size or a flap with a different size main element, may be able to established more precise scaling guidelines. 


\subsection{Effects of Angle of Attack}

The majority of data discussed so far is focused on various configurations at zero incidence. Although significant improvements are achieved at $\alpha=0^{\circ}$, a change of incidence adds another dimension to possible configurations and may provide even larger lift coefficients more effectively and efficiently. In the previous chapter the flap configuration $40 \mathrm{~A}$ is found to yield the highest lift most effectively with actuation from $7 \% c_{\text {flap }}$ and at $\Delta z=1$ in. Therefore, the discussion of incidence is focused on this configuration. Since actuation is applied far downstream of the model's leading edge, no significant delay of stall is achieved. This is exemplified in Chapter 4.3 (Figure 12) and consistently observed for all flap sizes, flap deflections, and actuation locations. The main goal of actuation is to prevent flow separation over the flap as $\alpha$ increases. Therefore, the actuation locations that are most effective for $\alpha=0^{\circ}$ do not change with incidence.

Instead of evaluating numerous $\alpha$-sweeps for different flap deflections, a more global and comprehensive approach is chosen. Similar to the methodology applied for $\alpha=0^{\circ}$, the entire data set is scanned for particular lift values and the respective combination of momentum input, flap deflection, and incidence at which the desired lift is achieved at a minimum corrected drag. The results obtained with actuation are compared to the baseline values. Figure 52 presents this data for drag corrections based on the momentum input. Compared to the baseline the maximum achievable lift increases from 1.7 to 2.95 when applying actuation. The maximum corrected lift-to-drag ratio (i.e., 
$\eta_{C_{\mu}}$ ) is more than doubled from 51 to 108 . The lift at which these values are achieved also increases from 0.7 to 1.7 in combination with an increase in flap deflection (from $10^{\circ}$ to $30^{\circ}$ ) and decrease in incidence (from $6.5^{\circ}$ to $0^{\circ}$ ). Not only does actuation increase the peak in $\eta_{C_{\mu}}$, it also sustains this high performance over a wide range of lift values (from $C_{l, d e s}=1.4$ to 2.4 ). This behavior would be beneficial for an aircraft's flight control. Flying at optimal performance would be less sensitive to variations in incidence, flap deflection, and flight speed. Although the required momentum input is increasing with $C_{l, d e s}$, the resulting $\eta_{C_{\mu}}$ is maintained at high levels. Note that for an optimal performance, the desired lift values are mostly attained at higher flap deflections and smaller angles of attack than for the baseline. This has significant implications for certain flight scenarios (e.g., take-off) and the corresponding limitations in aircraft design. For increasing lift values, the consistent behavior between $C_{\mu}, \delta_{T S}$, and $\alpha$ is also noteworthy. At a constant flap deflection, $\alpha$ continuous to increase before dropping again as $\delta_{T S}$ increases. The optimal combination of incidence, flap deflection, and actuation to yield the smallest corrected drag may be improved even further by refining the data set with intermediate values. 


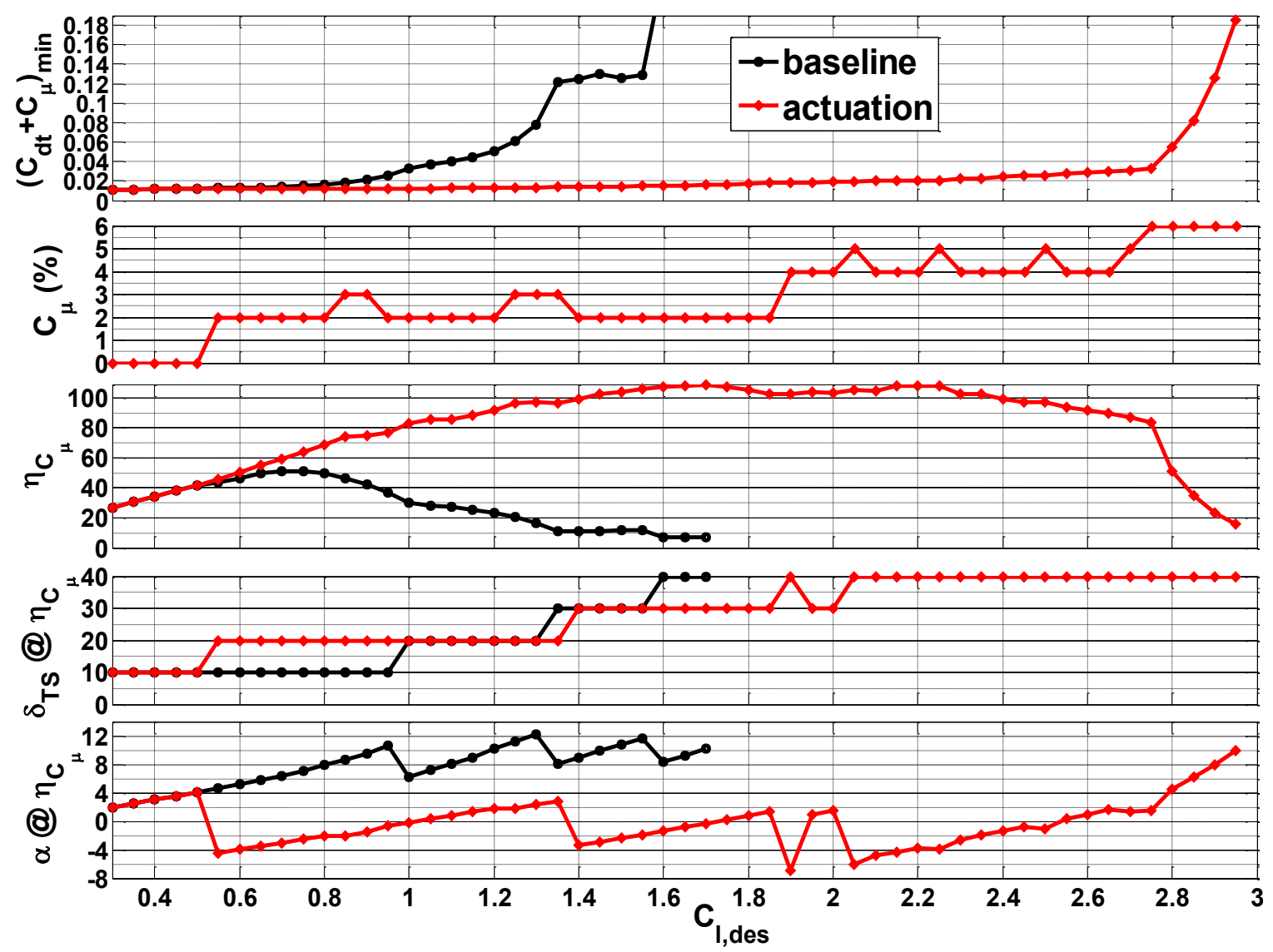

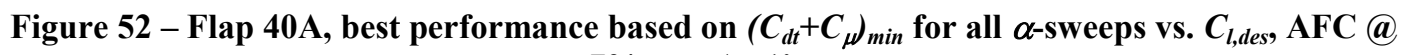
$7 \% c_{\text {flap }}, \Delta z=1$ in.

Although Figure 52 reveals substantial benefits by controlling separation with sweeping jets, for practical purposes a safety margin for $\alpha$ is considered. An aircraft has to fly at angles of attack sufficiently smaller than at which the maximum lift occurs. A typical safety margin includes $20 \%$ in velocity, which means that the maximum applicable lift coefficient corresponds to $C_{l, \max , \text { safe }}=C_{l, \max } / 1.44$. Considering only incidences which fall within the safety margin (Figure 53) reduces the maximum lift from 1.7 to 1.15 for the baseline and from 2.95 to 2.05 for actuation cases. However, the range of lift values remains almost doubled and only a few lift values close to $C_{l, \max }$ are obtained at a non-optimal configuration. The maximum value for $\eta_{C_{\mu}}$ is unchanged and 
can still be maintained over a wide range of $C_{l, d e s}$. The corrected drag does not change significantly with $C_{l, d e s}$, yielding an almost linear increase in $\eta_{C_{\mu}}$. Therefore, the best performance is obtained close to $C_{l, \max }$ which is desirable for certain flight scenarios (e.g., take-off).

Note that with the consideration of a safety margin, some of the maximum performance values presented for $\alpha=0^{\circ}$ (Chapter 6.3) may be reduced because at high flap deflections $\alpha_{\text {safe }}$ may be smaller than zero.
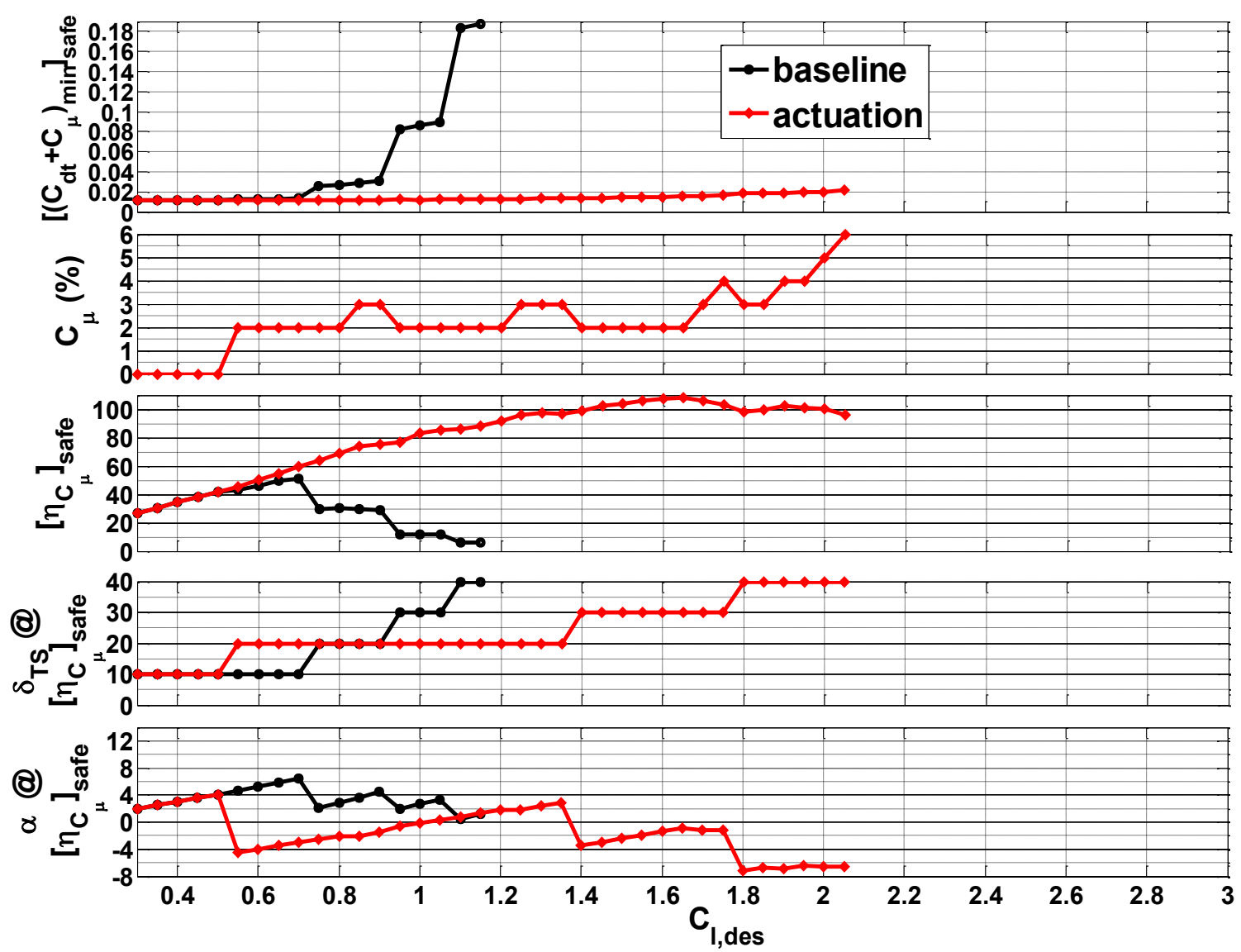

Figure 53 - Flap $40 \mathrm{~A}$, best performance based on $\left[\left(C_{d t}+C_{\mu}\right)_{\min }\right]_{\text {safe }}$ for all $\alpha$-sweeps with $20 \%$ safety margin in $C_{l, \max }$ vs. $C_{l, d e s}$, AFC @ 7\% $c_{\text {flap }}, \Delta z=1$ in.

The data presented in Figure 52 and Figure 53 are based on correcting the drag by the inserted momentum. However, the data may also be evaluated based on the input 
power coefficient, $C_{E}$. Since $C_{E}$ also accounts for the velocity ratio (Equation (10)), the resulting drag corrections are significantly larger than with $C_{\mu}$ (Figure 54). Therefore, the smallest corrected drag is found for noticeably smaller values of $C_{\mu}$ in conjunction with smaller flap deflections and larger angles of attack (cf. Figure 52). The subsequent lift-todrag ratios (i.e., $\eta_{C_{E}}$ ) are not as optimistic as for $\eta_{C_{\mu}}$ In fact, the maximum $\eta_{C_{E}}$ coincides with the baseline value. However, as the baseline performance declines, actuation is able to maintain a high performance level with increasing $C_{l, d e s}$. A second local maxima for $\eta_{C_{E}}$, which is just slightly smaller than the global maxima, is achieved at $C_{l, d e s}=1.7$. This indicates that when accounting for actuation at a high expenditure, active flow control becomes only efficient once the baseline potential is exhausted. Then, actuation is able to maintain at least a comparable level of performance for significantly higher lift values.

The discussion of a safety margin for $\alpha$ may be repeated for the data in Figure 54. However, this plot is omitted because similar observations as in Figure 53 are revealed with the same reduction in $C_{l, \max }$ and the maintained regime of high performance when applying actuation.

The evaluation of efficiency based on either $C_{\mu}$ or $C_{E}$ leads to considerably different performance levels and geometric configurations. This indicates that any optimization process for specific applications strongly depends on performance goals and efficiency criteria. 

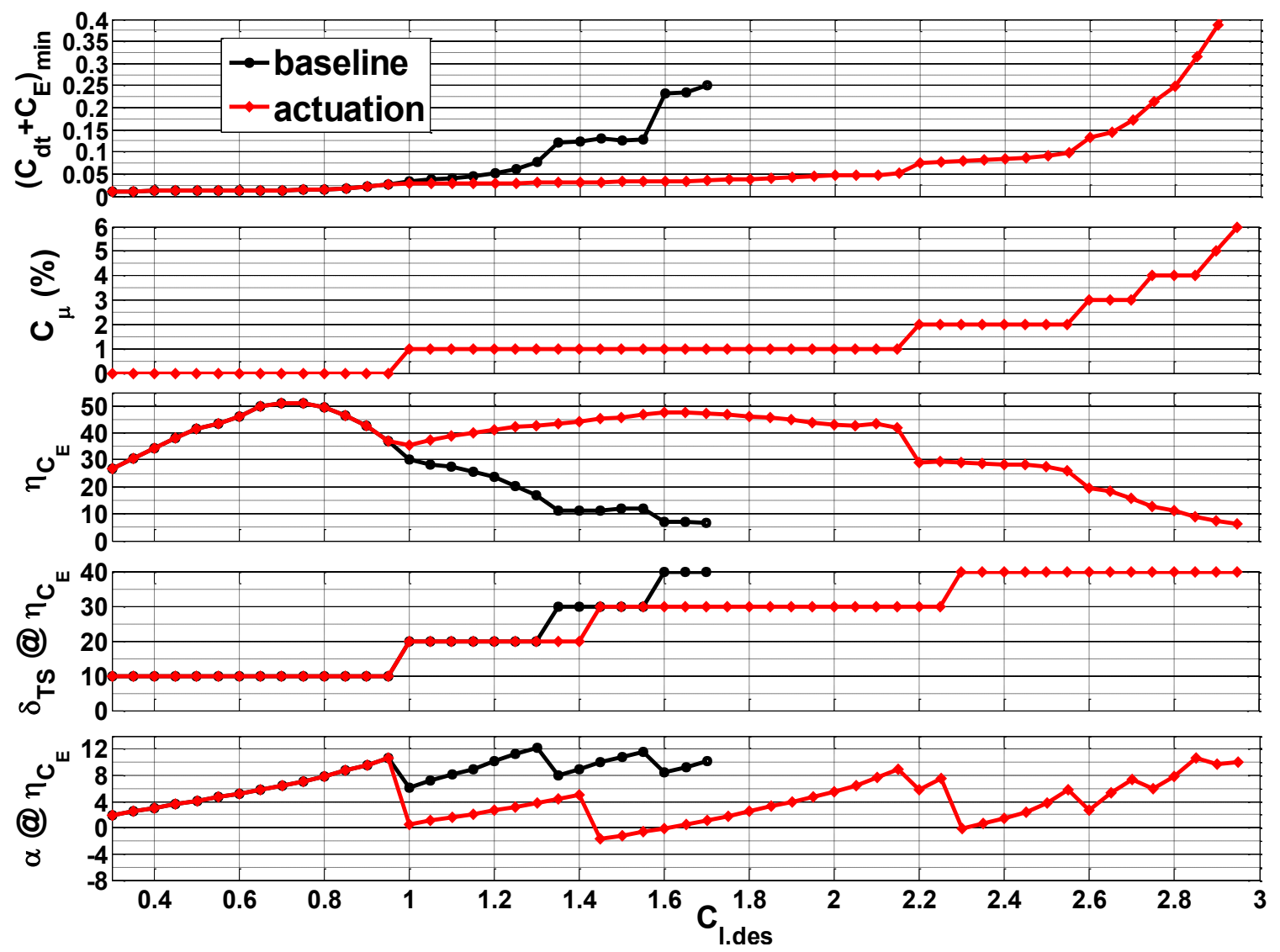

Figure 54 - Flap 40A, best performance based on $\left(C_{d t}+C_{E}\right)_{\min }$ for all $\alpha$-sweeps vs. $C_{l, d e s}$, AFC @ $7 \% c_{\text {flap }}, \Delta z=1$ in. 


\section{HOW CAN ACTUATION BE SCALED?}

The data presented in the previous chapters indicate certain trends and dependencies for the effective and efficient use of sweeping jets for separation control over a trailing edge flap. Based on the available information this chapter proposes a possible strategy to scale and optimize sweeping jet actuators for full-size applications.

Before examining the scaling process, the first step is aimed at defining the objectives and requirements for a specific application. These objectives may either be based on obtaining the most improvements from a fixed geometry or on achieving a specific performance (e.g., a desired $C_{l}$ or $L / D$ ) through the use of sweeping jet actuators. The first objective is certainly easier to pursue because the geometry is already determined. More extensive preparatory work is necessary for the second objective. The discussion in Chapter 6.1 suggests that potential flow solutions are a suitable tool to estimate the efficiently achievable lift from a specific geometry. If a desired performance is not predicted by potential flow solution, the use of sweeping jets may not yield this performance in an efficient manner either. In addition, three-dimensional effects (e.g., tip vortex) may reduce the estimated performance even further. Therefore, a proposed model size and shape need to inherit at least the necessary geometric potential to yield intended values. Chapter 6 outlines the effects of various geometric parameters (i.e., flap deflection angle, flap size, and angle of attack) which are fundamental for the achievable lift. Considering that the angle of attack is often desired to be close to zero (e.g., for a vertical tail), the ideal flap size has to be determined in conjunction with the flap 
deflection. As argued in Chapter 6.3 that the smallest suitable flap size yields the smallest corrected drag for a desired lift, even though higher flap deflections are required.

With the initial proposal of a specific geometry, an iterative process is necessary in conjunction with system requirements and limitations. These considerations include several application specific factors. For example, the available flowrate for a full-size aircraft has to be determined in order to estimate available values of $C_{\mu}$ and $C_{Q}$. Further, geometric and safety limitations (e.g., in angle of attack and flap deflection) also have to be considered. Requirements and restrictions specific to the intended mission statement (e.g., take-off and landing procedures, maximum and minimum flight speeds, payload, range, endurance, etc.) also affect the choice of geometry. Besides the desired lift, the moments often play an important role which can also be predicted by inviscid flow solutions. For example, the control of pitching moment for BWB applications is a crucial part of the wing and flap design. Structural considerations (e.g., load distribution, stresses, weight, etc.) may also be estimated from potential flow solutions.

Once a suitable geometry and range of motion (e.g., for flap deflection) is proposed, either through an existing concept or by design, the application of flow control has be to scaled and optimized specific to the application. This process may either be accomplished with a small-scale or full-scale model, which allows the necessary flexibility in changing desired parameters. Although more expensive and complex, tests under conditions as close to full-scale will likely yield the most valuable information for full-scale applications. 
The first parameter to be established is the actuation location. The arguments presented in Chapter 5 indicate that actuation should be applied from a single location on the flap close to the flap's leading edge but downstream of highly curved sections of the flap. Although the range of locations tested in the current work is coarse, no dependence of actuation location on $C_{\mu}, \Delta z$, or $\delta_{T S}$ is evident. Therefore, no detailed knowledge of these parameters is required for the search of an ideal actuation location. In fact, it is argued in Chapter 5.1 that with actuation at a non-ideal configuration and at high flap deflection, the differences between various locations are more pronounced and therefore, more easily identified. However, the effects of exit angle and curvature on actuation location may be of importance and should be investigated in separate experiments. Although two rows of actuation may be an option, Chapter 5.2 illustrates that two rows are not as effective and efficient as a single row. However, due to the possible limitation in exit velocity comparable values of $C_{\mu}$ and $C_{Q}$ may be achieved by a second row instead of further enlarging the actuators in a single row. Also, a second actuation location further downstream on the flap may enhance the streamwise penetration if necessary.

The third step in applying sweeping jets successfully on a full-scale application is the determination of the spacing between adjacent actuators. As suggested throughout the current work (e.g., Chapters 4.4 and 4.6) the sweeping jets require a continuous impact area along the span to be effective. The goal is to increase the actuators' spacing as much as possible without losing effectiveness. The largest possible spacing requires the least flowrate and provides the highest momentum input which is directly linked to the 
reduction in drag. The spanwise coverage of the flap is defined by the sweeping properties of the actuators (i.e., sweeping range, $\theta_{\text {sweep }}$ ) and the counteracting bending effect of the free-stream, thereby providing a fairly small range for $\Delta z$. If tests on a smallscale model establish a suitable value for $\Delta z$ for particular actuators, this value is likely not to be exceeded at higher free-stream velocity because the increased bending effect reduces spanwise coverage. Retracting the actuators into a slot may increase $\Delta z$ because a larger spanwise spreading of the jets is allowed before interacting with the free-stream. The sweeping angle of a particular actuator is given by its geometry and not by its size. Only the increase in outlet width adds to the covered flap area which may be offset by the increase in free-stream velocity for a full-scale application. Therefore, scaling the model's and actuator's size is expected to be independent of $\Delta z$. Recent experiments ${ }^{[69]}$ with altered actuators, which inherit a larger sweeping angle, yielded comparable success in controlling flow separation with a larger actuator spacing. However, increasing the sweeping angle results in less average momentum injected in $x$-direction (Chapter 4.1), which may lead to loss of streamwise penetration. Appropriate values of $\theta_{\text {sweep }}$ and $\Delta z$ in conjunction with various actuator geometries can be investigated in simplified experiments without requiring the actual application model in full scale.

Once a suitable actuator location, geometry, and spacing are chosen the last step is aimed at scaling the actuator's size. Assuming that the jet velocity is bound by sonic speed, the necessary actuator size, and therefore flowrate, can be estimated in order to obtain comparable values of $C_{\mu}$. Here, a larger outlet width than height may be desirable to maximize spanwise coverage and simplify the actuators' integration into a model. For 
traditional actuation methods the importance of $U_{\text {jet }} / U_{\text {inf }}$ has been indicated ${ }^{[7,9]}$. However, due to different underlying mechanisms for separation control with sweeping jets, the role of $U_{j e t} / U_{\text {inf }}$ has yet to be determined. The strength of the suggested streamwise vortices is not just governed by the jets' velocity, but by their flowrate as well. Nevertheless, it seems advisable that the jet velocity is kept as high as possible. The scaling efforts for a desired jet velocity and flowrate are mainly governed by the actuator's main chamber dimensions (i.e., chamber inlet and outlet size) and not by the size and geometry of the feedback loops. Arwatz et al. ${ }^{[46]}$ illustrated that an increase in length of the feedback loop reduces the sweeping frequency, whereas an increase in cross-sectional area of the feedback loop increases the sweeping frequency. It has been shown that the actuators used in this study yield a sweeping frequency smaller by an order of magnitude if every dimension is scaled by only a factor of five. If based on current wind tunnel experiments the dominant length scale (e.g., chord or flap length) and the free-stream velocity increase by a comparable factor, the sweeping frequency of the scaled actuators would have to be the same order of magnitude in order to maintain similar values of $F^{+}$. This may be achieved by appropriate design and dimensioning of the feedback loops. Tests of various actuator and feedback loop designs should be performed prior to the particular application experiments.

Depending on the performance of the sweeping jet actuators on a larger or fullscale model, the aforementioned steps may have to be repeated iteratively in order to meet defined objectives and system requirements. This process may be supported by prior computational efforts to reduce the number of iterations. In addition to the outlined 
scaling strategy, specific applications may require additional optimization efforts. For example, a tapered and swept wing may lead to a non-uniform actuator size along the span and the jets not exiting in streamwise direction. Further, geometric additions to the wing (e.g., winglets) may also increase the performance of sweeping jets. 


\section{CONCLUSIONS}

The current study addresses a multitude of parameters affecting the control of separation by an array of sweeping jet actuators distributed evenly along the span of a generic airfoil. The initial characterization of the actuators confirms that the emitted jets oscillate at high frequencies directly proportional to the volume flow rate provided through each actuator implying that a flow is present in the actuator's feedback channels. Minor deviations from the linear pattern are noticed consistently for various spacings between adjacent actuators; however, they cannot be explained yet. The jet velocity appears to be bound by sonic speed, close to which the sweeping frequency saturates at $\sim 5 \mathrm{kHz}$ for the current actuator geometry. In absence of a free-stream, thrust measurements demonstrate that between $80 \%$ and $90 \%$ of the total jet momentum is bound in $x$-direction almost independent of the actuator spacing and without significant losses as sonic speed is approached. This is mainly due to the jets' off-center excursions as correctly predicted by a simplified mathematical model assuming the sweeping motion of the jet may be approximated by a sine wave. However, in the presence of a free-stream the recovery of the total jet momentum in thrust is found to be consistently close to $100 \%$, implying that the off-center excursions of the sweeping jets are hampered by the free-stream, eventually bending them in $x$-direction. Experimental data from separation control with various $\Delta z$ reveal that for actuation to be effective the maximum distance between adjacent actuators is limited. Therefore, based on the ratio between jet and freestream velocity the actuator spacing ought to be chosen in a way that the sweeping 
motion still provides a continuous impact area along the span of a surface to be controlled. Variations in the actuators' design may lead to other optimal values for $\Delta z$.

For a fixed actuator spacing, the momentum coefficient governs actuation with sweeping jets for different free-stream velocities. However, if $\Delta z$ changes, neither the flowrate coefficient nor the momentum coefficient can collapse the data. Nevertheless, the product of the two (i.e., $\left.\left(0.5 C_{\mu}\right)^{0.5} C_{Q}\right)$ yields good agreement for various actuator spacings over a wide range of flap deflections and flap sizes. This coefficient also suggests that the effectiveness of this actuation method is independent of sweeping frequency when being of the order of $\mathrm{kHz}$.

The investigation of hysteresis effects reveals that no hysteresis with respect to changes in actuation intensity or flap deflection is evident. Therefore, the application of sweeping jets is simplified because no complex sequencing or closed-loop control mechanisms are necessary to achieve a desired performance. The absence of hysteresis also implies that separation control with sweeping jets not only inherits its effectiveness from the inserted momentum, but from beneficial structures that are formed. This is supported by surface flow visualization results which reveal the formation of vortex pairs in between adjacent actuators. The vortices are located at the interaction zone between neighboring jets. The initially normal vorticity is bent by the free-stream to form pairs of streamwise vortices that are retained all the way to the flap's trailing edge. The vortices maintain flow attachment by removing low momentum fluid from the boundary layer and supply it with high momentum fluid from the outer region. A quantitative and qualitative comparison between sweeping and non-sweeping jets, originating from the same size 
actuators, supports these arguments. Although non-sweeping jets also appear to form streamwise vortices, separation control with sweeping jets is superior because the sweeping motion provides larger area coverage and thereby more effective control.

The effects of actuation location on momentum input is found to be well described by the coefficient $C_{\mu} \cdot\left(100-x_{a c t} / c_{f l a p}\right)^{0.5}$, implying that the further actuation is applied away from the flap shoulder, the more momentum is required to attach the flow. However, this argument only holds for actuation applied from the straight section of the flap due to geometric effects at the flap shoulder. Although the exit angle of the jets, emitted from the trailing of the main element, increases with flap deflection, the main reason for the reduced effectiveness of actuation from this location is found to be the downstream curvature of the flap's leading edge. This also suggests that exit angles significantly larger than the current $30^{\circ}$ may be worth investigating in future experiments. Within the coarse range of actuation locations tested, actuation applied at the most upstream location on the flap is most effective in controlling separation regardless of $\delta_{T S}$, $\alpha$, and $C_{\mu}$. The differences between various actuation locations are more pronounced at higher flap deflections and for less effective actuator spacings. A comparison between various flap sizes implies that the actuation location does not scale as a percentage of the flap's chord. Instead, an absolute downstream distance on the flap in reference to the model's total chord length provides better agreement. Additional parameters (e.g., actuator size, flap geometry, etc.) may be relevant as well. Future experiments may provide a finer resolution in actuation location to identify any further dependencies. Simultaneous actuation from two rows is also effective in controlling separation when 
maintaining similar values of $C_{\mu}$. However, a significantly higher total flowrate is required to yield results comparable to actuation from a single, most effective location. Although actuation from two rows of actuators introduces twice the amount of sweeping jets, the reduced strength of an individual jet is not compensated by the increased numbers.

The comparison between experimental and numerical data of the MFA model reveals that, in absence of flow separation from the flap's bottom surface, an inviscid flow solution is a good predictor for the efficiently achievable lift and moment. Any improvements beyond the prediction are marginal and require an inefficient increase in $C_{\mu}$. Therefore, potential flow theory could be a valuable tool to predict upper bounds for the improvements expected from the use of sweeping jet actuators which ultimately could aid the design and layout for specific applications.

For a fixed actuator spacing, the relationship of momentum input, actuation location, and flap deflection is described by the single coefficient $C_{\mu} \cdot\left(100-x_{a c t} / c_{f l a p}\right)^{0.5} / \delta_{T S}$. This coefficient yields a linear pattern for the differential increase in lift if $C_{\mu}$ is not increased beyond the threshold value at which the flow is fully attached. The same pattern is resembled by extending this coefficient to $\left(0.5 C_{\mu}\right)^{0.5} C_{Q}\left(100-x_{a c t} / c_{f l a p}\right)^{0.5} / \delta_{T S}$, which also accounts for various $\Delta z$. These coefficients summarize the statement that the actuation intensity required to attach the flow increases the more the flap is deflected and the further downstream actuation is applied on the flap.

The evaluation of flap size at $\alpha=0^{\circ}$ indicates that the momentum required to attach the flow over the flap at the same deflection is governed by the momentum coefficient 
based on the flap's chord length (i.e, $C_{\mu f f a p}$ ) and not on the total chord length (i.e., $C_{\mu}$ ). Therefore, scaling efforts for momentum input should be based on the length of the flap. Efficiency arguments based on corrected drag values suggest that a desired lift is most efficiently obtained with the smallest possible flap as long as that flap size inherits sufficient geometric potential to yield the desired lift at reasonable flap deflections. Including changes in incidence adds another dimension to find an optimal configuration for a desired performance. Significant improvements in corrected lift-to-drag ratio are obtained at smaller angles of attack and larger flap deflections which has significant implications for certain flight procedures and the respective limitations for aircraft design. For example, an aircraft may be able to take off and land at a smaller angle of attack which would alleviate the limitations for the shape of the fuselage's aft section. Although the range of achievable lift values is significantly reduced when considering a safety margin for the maximum applicable incidence, actuation still provides superior improvements to the baseline. Not only does actuation yield a significant increase in performance, it also maintains a high performance level over a wider range of lift values and geometric configurations. Therefore, an aircraft may perform at a desired level with less sensitivity to changes in incidence, flap deflection, and speed. The search for an optimal combination of geometry and actuation is strongly dependent on the efficiency criteria (e.g., $C_{\mu}$ or $C_{E}$ ) and the desired performance goals (e.g., $C_{l, \max }$ or $\eta_{\max }$ ).

The findings provided by this study are comprised in a proposal of how to scale and optimize the use of sweeping jets for a particular application. Although this actuation method is very promising due to its simplicity and effectiveness, its investigation and 
understanding is in its early stages. Important questions and detailed understanding of the underlying physics remain to be addressed (see Chapter 9) in order to successfully employ separation control with sweeping jet actuators to full-scale applications. 


\section{FUTURE WORK}

Although this study covers a wide spectrum of parameters affecting separation with sweeping jets, it raises more questions than it answers. This is expected because the use of sweeping jets for active flow control purposes has only been investigated for a few years whereas more traditional flow control methods have been studied in detail for several decades. Therefore, some future experiments targeted at verifying, understanding, and improving the arguments presented in the current work are outlined in this chapter.

In order to streamline future efforts, it may be desirable to order the investigated parameters by significance. However, it is challenging to rank the importance of an individual parameter due the interdependence between most of them. The list shown below identifies three leading groups of parameters which appear to hold the most impact on the effective and efficient application of sweeping jets.

$$
C_{l}=C_{l}\left[(\text { model geometry }) ;\left(\Delta z, \frac{U_{\text {jet }}}{U_{\text {inf }}}\right) ;\left(x_{\text {act }}\right)\right]
$$

Several other parameters are involved in the investigation and optimization of these individual groups. The model's geometry (e.g., thickness, camber, flap size, shape and deflection, angle of attack, etc.) is a crucial factor in providing the necessary potential to yield a desired lift and to meet application specific requirements. The second group presents an essential part of effective and efficient actuation with sweeping jets because they govern important coefficients such as $C_{\mu}, C_{Q}$, and $C_{E}$. Their optimization requires a detailed study of sweeping jet actuators and their properties which is outlined in the following paragraphs. The third essential parameter is the actuation location which 
also has a significant impact on the actuation's effectiveness and efficiency. Factors such as local geometry and exit angle have to be addressed in more detail to optimize this parameter.

One of the main goals for future research should be the continued investigation and development of sweeping jet actuators optimized for separation control. Most of this work can be conducted in absence of a free-stream. For example, a large-scale single actuator should be examined to gain a better understanding of the internal mechanisms, governing parameters, and how they can be altered to yield desirable characteristics which include a high sweeping frequency, large sweep angle, and reduced internal losses (e.g., shocks and separation in the feedback loop). The study of a single actuator also has to address the question of jet velocity and its possible limitations. Changes in geometry may avoid internal and external shocks and yield different maximum jet velocities. In conjunction with developing specific actuators, the flow physics of sweeping jets in the presence of a free-stream with and without a pressure gradient have to be examined on a time resolved scale. This may also include the study of exit angle to the free-stream. Initial experiments should be based on a single actuator before extending the interest to an array of actuators at various distances between them. The study of an array of actuators could include the development of phase controlled sweeping motion which may be beneficial for certain applications (e.g., film-cooling).

In addition to the investigation and development of sweeping jet actuators, the gained understanding has to be applied to separation control scenarios. Experiments on larger scale models and higher free-stream velocities will be able to answer important 
questions regarding scaling efforts. One of the most imperative questions to be answered pertains to the effect of $U_{j e t} / U_{\text {inf }}$ and whether reduced velocity ratios can be balanced by increased actuator size and flowrate. If supersonic jet velocities are attainable, it remains to be answered whether they are beneficial for separation control at subsonic free-stream velocities. Another important aspect that is not sufficiently addressed in this study is the effect of jet exit angle. The work of other researchers ${ }^{[12,60]}$ suggests different optimal exit angles ranging from $0^{\circ}$ to more than $90^{\circ}$. In conjunction with exit angle the possible interdependence of flap deflection and actuation location warrants detailed experiments to increase the actuators effectiveness and efficiency.

The significant conclusion that the momentum coefficient based on the flap's chord is a more appropriate governing parameter than $C_{\mu}$ based on the total chord length has to be verified. In this study this conclusion is based on various flap sizes with different geometries and therefore different pressure gradients. This claim should be validated either with a small and large size model with the same geometry and flap chord percentage or with the same size flap and different size main elements. In order to bypass scaling issues of the actuators for different size models, the experiments could be controlled by Reynolds number.

Other experiments related to general separation control applications may include the recess of actuators in the flap to allow for a more spanwise spreading of the jets before interacting with the outside flow. Additional studies could include unsteady changes in flap deflection and/or incidence to further examine questions of hysteresis and 
control authority. Besides separation control experiments, sweeping jet actuators could also be tested for other purposes such as noise reduction and film cooling.

The knowledge gained from fundamental experiments can be applied to particular applications, which would require additional optimization efforts. Since the study of separation control with sweeping jets has mostly focused on separation over the flap, the delay of stall over a model's main element offers other possible applications (e.g., fixed wings at high incidence, wind turbines, helicopters, or any rotating blade applications). The delay of leading edge stall may even be combined with separation control over a trailing edge flap. Applications, which are currently investigated, include the vertical tail of a commercial aircraft. Here, three-dimensional effects, such as the sweep and taper of the tail, require additional optimization. For example, due to the taper, the size of the actuators and their air supply may change along the span.

The proposed efforts will hopefully result in the use of sweeping jets on full-scale applications in the near future and thereby, prove the value of these devices as a tool for active flow control. 


\section{REFERENCES}

1. Prandtl, L., "Über Flüssigkeitsbewegung bei sehr kleiner Reibung", Verhandlungen des dritten internationalen Mathematischen Kongressen, Heidelberg, Germany, Teubner Verlag, Leipzig, Germany, 1904, pp. 484-491.

2. Zahkarin, B., Woszidlo, R., and Wygnanski, I., "On the Significance of Streamwise Vorticity in Turbulent Mixing and Separation Control Applications", International Conference on Jets, Wakes and Separated Flows, 16-19 Sept., Technical University of Berlin, Germany, 2008.

3. Woszidlo, R., Taubert, L., and Wygnanski, I., "Manupulating the Flow over Spherical Protuberances in a Turbulent Boundary Layer", AIAA Journal, doi: 10.2514/1.39930, Vol. 47, No. 2, Feb. 2009, pp. 437-450.

4. Richter, K. and Rosemann, H., "Steady Aerodynamics of Miniature Trailing-Edge Devices in Transonic Flows", AIAA 2011-3354, AIAA $29^{\text {th }}$ Applied Aerodynamics Conference, 27-30 June, Honolulu, HI, 2011.

5. Seifert, A., Bachar, T., Koss, D., Shepshelovich, M., and Wygnanski, I., Oscillatory Blowing: A Tool to Delay Boundary Layer Separation", AIAA Journal, doi: 10.2514/3.49121, Vol. 31, No. 11, Nov. 1993, pp. 2052-2060.

6. Seifert, A. and Pack, L.G., "Oscillatory Control of Separation at High Reynolds Numbers", AIAA Journal, doi: 10.2514/2.834, Vol. 37, No. 9, Sept. 1999, pp. 1062 1071.

7. Nagib, H., Kiedaisch, J., Reinhard, P., and Demanett, B., "Control Techniques for Flows with Large Separated Regions: A New Look at Scaling Parameters", AIAA 2006-2857, AIAA $3^{\text {rd }}$ Flow Control Conference, 5-8 June, San Francisco, CA, 2006.

8. Cerchie, D., Halfon, E., Hammerich, A., Han, G., Taubert, L., Trouve, L., Varghese, P., and Wygnanski, I., "Some Circulation and Separation Experiments", Applications of Circulation Control Technology, Joslin, R. and Jones, G. (editors), Progress in Astronautics and Aeronautics Series, Vol. 214, published by AIAA, 2006.

9. Chen, C., "Separation and Circulation Control on a Thick Blunt Elliptical Airfoil", Dissertation (Ph.D.), University of Arizona, Tucson, AZ, 2009.

10. Crowther, W.J., "Separation Control on a Trailing-Edge Flap Using Air Jet Vortex Generators", AIAA Journal of Aircraft, doi: 10.2514/1.15755, Vol. 43, No. 5, September-October 2006, pp. 1589-1593. 


\section{REFERENCES - Continued}

11. Kumar, V. and Alvi, F.S., "Use of High-Speed Microjets for Active Separation Control in Diffusers", AIAA Journal, doi: 10.2514/1.8552, Vol. 44, No. 2, Feb. 2006, pp. 273-281.

12. Kumar, V., "Separation Control in Adverse Pressure Gradients Using High-Speed Microjets", Dissertation (Ph.D.), Florida State University, Tallahassee, FL, 2008.

13. Kumar, V. and Alvi, F.S., "Toward Understanding and Optimizing Separation Control Using Microjets", AIAA Journal, doi: 10.2514/1.38868, Vol. 47, No. 11, Nov. 2009, pp. 2544-2557.

14. Mabe, J., Calkins, F., Wesley, B., Woszidlo, R., Taubert, L., and Wygnanski, I., "Single Dielectric Barrier Discharge Plasma Actuators for Improved Airfoil Performance", AIAA Journal of Aircraft, doi: 10.2514/1.37638, vol. 446, No. 3, MayJune 2009, pp. 847-855.

15. Nishri, B. and Wygnanski, I., "Effects of Periodic Excitation on Turbulent Flow Separation from a Flap", AIAA Journal, doi: 10.2514/2.428, Vol. 36, No. 4, April 1998, pp. 547-556.

16. Seifert, A., Eliahu S., Greenblatt, D., and Wygnanski, I., "Use of Piezoelectric Actuators for Airfoil Separation Control", AIAA Journal, doi: 10.2514/2.549, Vol. 36, No. 8, Aug. 1998, pp. 1535-1537.

17. Greenblatt, D. and Wygnanski, I., "Dynamic Stall Control by Periodic Excitation, Part 1: NACA 0015 Parametric Study", AIAA Journal of Aircraft, doi: 10.2514/2.2810, Vol. 38, No. 3, May-June 2001, pp. 430-438.

18. Greenblatt, D., Nishri, B., Darabi, A, and Wygnanski, I., "Dynamic Stall Control by Periodic Excitation, Part 2: Mechanism", AIAA Journal of Aircraft, doi: 10.2514/2.2811, Vol. 38, No. 3, May-June 2001, pp. 430-438.

19. Wygnanski, I., "The Variables Affecting the Control of Separation by Periodic Excitation", AIAA 2004-2505, AIAA $2^{\text {nd }}$ Flow Control Conference, 28 June - 1 July, Portland, OR, 2004.

20. Darabi, A. and Wygnanski, I., "Active management of naturally separated flow over a solid surface. Part 1. The forced reattachment process", Journal of Fluid Mechanics, doi: 10.1017/S0022112004009231, Vol. 510, 2004, pp.105-129.

21. Darabi, A. and Wygnanski, I., "Active management of naturally separated flow over a solid surface. Part 2. The separation process", Journal of Fluid Mechanics, doi: 10.1017/S0022112004009243, Vol. 510, 2004, pp.131-144. 


\section{REFERENCES - Continued}

22. Glezer, A., Amitay, M., and Honohan, A.M., "Aspects of Low- and High-Frequency Actuation for Aerodynamic Flow Control", AIAA Journal, doi: 10.2514/1.7411, Vol. 43, No. 7, July 2005, pp. 1501-1511.

23. Schmalzel, M., Varghese, P., and Wygnanski, I., "Download Alleviation on a V-22 Model Having a Simple Flap Used in Conjunction with Periodic Excitation, Suction, and Blowing", International Powered Lift Conference, Society of Automotive Engineers, doi: 10.4271/2005-01-3188, 2005.

24. Gad-el-Hak, M., "Flow Control: The Future", AIAA Journal of Aircraft, doi: 10.2514/2.2796, Vol. 38, No. 3, May-June 2001, pp. 402-418.

25. Kibens, V., and Bower, W.W., "An Overview of Active Flow Control Applications at the Boeing Company", AIAA 2004-2624, AIAA $2^{\text {nd }}$ Flow Control Conference, 25 June-1 July, Portland, OR, 2004.

26. Collis, S.S., Joslin, R.D., Seifert, A., and Theofilis, V., "Issues in Active Flow Control: Theory, Control, Simulation, and Experiment", Progress in Aerospace Science, doi: 10.1016/j.paerosci.2004.06.001, Vol. 40, Issues 4-5, May-July 2004, pp. 237-289.

27. Wygnanski, I., "A Century of Active Control of Boundary Layer Separation: A Personal View", IUTAM Symposium on One Hundred Years of Boundary Layer Research, Meier, G.E.A and Sreenivasan, K.R. (editors), doi: 10.1007/978-1-40204150-1_15, Volume 129, Session 3, Springer Verlag, 2006, pp. 155-165.

28. Anders, S.G., Sellers III, W.L., and Washburn, A. E., "Active Flow Control Activities at NASA Langley", AIAA 2004-2623, AIAA $2^{\text {nd }}$ Flow Control Conference, 25 June-1 July, Portland, OR, 2004.

29. Cattafesta III, L.N. and Sheplak, M., "Actuators for Active Flow Control", Annual Review of Fluid Mechanics, doi: 10.1146/annurev-fluid-122109-160634, Vol. 43, Issue 1, Aug. 2010, pp. 247-272.

30. Spalart, P.R. and McLean, J.D., "Drag reduction: enticing turbulence, and then an industry", Philosophical Transactions of the Royal Society - Series A: Mathematical, Physical and Engineering Sciences, doi: 10.1098/rsta.2010.0369, Vol. 369, Issue 1940, April 2011, pp. 1556-1569.

31. Viets, H., "Flip-Flop Jet Nozzle", AIAA Journal, doi: 10.2514/3.60550, Vol. 13, No. 10, Oct. 1975, pp. 1375-1379. 


\section{REFERENCES - Continued}

32. Srinivas, T. Vasudevan, B., and Prabhu, A., "Performance of Fluidically Controlled Oscillating Jet", Turbulence Management and Relaminarization, Proceedings of the IUTAM Symposium, Bangalore, India, 13-23 Jan. 1987 (A89-10154 01-34), Springer Verlag, Berlin and New York, 1988, pp. 485-494.

33. Gregory, J.W., Sullivan, J.P., Raman, G., and Raghu, S., "Characterization of a Micro Fluidic Oscillator", AIAA Journal, doi: 10.2514/1.26127, Vol. 45, No. 3, March 2007, pp. 568-576.

34. Stouffer, R.D., "Oscillating Spray Device", U.S. Patent 4,151,955, issued May 1, 1979.

35. Bray Jr., H.C., "Cold Weather Fluidic Fan Spray Devices and Method", U.S. Patent 4,463,904, issued Aug. 7, 1984.

36. Cerretelli, C. and Kirtley, K., "Boundary Layer Separation Control with Fluidic Oscillators", Journal of Turbomachinery, doi: 10.1115/1.3066242, Vol. 131, Oct. 2009.

37. Seele, R., Tewes, P., Woszidlo, R., McVeigh, M., Lucas, N., and Wygnanski, I., "Discrete Sweeping Jets as Tools for Improving the Performance of the V-22", AIAA Journal of Aircraft, doi: 10.2514/1.43663, Vol. 46, No. 6, 2009, pp. 2098-2106.

38. Raman, G. and Raghu, S., "Miniature Fluidic Oscillators for Flow and Noise Control: Transitioning from macro to micro fluidics", AIAA 2000-2554, AIAA Fluids 2000 Conference and Exhibit, 19-22 June, Denver, CO, 2000.

39. Raman, G. and Raghu, S., "Cavity Resonance Suppression Using Miniature Fluidic Oscillators", AIAA Journal, doi: 10.2514/1.521, Vol. 42, No. 12, Dec. 2004, pp. 2608-2611.

40. Guyot, D., Bobusch, B., Paschereit, C.O., and Raghu, S., "Active Combustion Control Using a Fluidic Oscillator for Asymmetric Fuel Flow Modulation", AIAA 2008-4956, 44 ${ }^{\text {th }}$ AIAA/ASME/SAE/ASEE Joint Propulsion Conference \& Exhibit, Hartford, CT, July 2008.

41. Raman, G., Hailye, M., and Rice, E.J., "Flip-Flop Jet Nozzle Extended to Supersonic Flows", AIAA Journal, doi: 10.2514/3.12444, Vol. 31, No. 6, June 1993, pp. 10281035.

42. Raman, G., "Using Controlled Unsteady Fluid Mass Addition to Enhance Jet Mixing", AIAA Journal, doi: 10.2514/2.185, Vol. 35, No. 4, April 1997, pp. 647-656. 


\section{REFERENCES - Continued}

43. Gregory, J.W., Sullivan, J.P., and Raghu, S., "Visualization of Jet Mixing in a Fluidic Oscillator", Journal of Visualization, doi: 10.1007/BF03181660, Vol. 8, No. 2, 2005, pp. 169-176.

44. Culley, D.E., "Variable Frequency Diverter Actuation for Flow Control", AIAA 20063034, AIAA $3^{\text {rd }}$ Flow Control Conference, 5-8 June, San Francisco, CA, 2006.

45. Gregory, J.W., Ruotolo, J.C., Byerley, A.R., and McLaughlin, T.E., "Switching Behavior of a Plasma-Fluidic Actuator", AIAA 2007-785, 45 th AIAA Aerospace Sciences Meeting and Exhibit, Reno, NV, Jan. 2007.

46. Arwatz, G., Fono, I., and Seifert, A., "Suction and Oscillatory Blowing Actuator Modeling and Validation", AIAA Journal, doi: 10.2514/1.30468, Vol. 46, No. 5, May 2008, pp. 1107-1117.

47. Gregory, J.W., Gnanamanickam, E., Sullivan, J., and Raghu, S., "Variable-Frequency Fluidic Oscillator Driven by a Piezoelectric Bender", AIAA Journal, doi: 10.2514/1.44078, Vol. 47, No. 11, Nov. 2009, pp. 2717-2725.

48. Cerretelli, C. and Gharaibah, E., "An Experimental and Numerical Investigation on Fluidic Oscillators For Flow Control", AIAA 2007-3854, AIAA 37 $7^{\text {th }}$ Fluid Dynamics Conference and Exhibit, 25-28 June, Miami, FL, 2007.

49. Feikema, D. and Culley, D., "Computational Fluid Dynamic Modeling of a Fluidic Actuator for Flow Control", AIAA 2008-557, AIAA 46 ${ }^{\text {th }}$ Aerospace and Sciences Meeting and Exhibit, 7-10 Jan., Reno, NV, 2008.

50. Gokoglu, A.S., Kuczmarski, M.A., Culley, D.E., and Raghu, S., "Numerical Studies of Fluidic Diverter for Flow Control", AIAA 2009-4012, AIAA $39^{\text {th }}$ Fluid Dynamics Conference, 22-25 June, San Antonio, TX, 2009.

51. Gokoglu, S.A., Kuczmarski, M.A., Culley, D.E., and Raghu, S., "Numerical Studies of a Supersonic Fluidic Diverter Actuator for Flow Control", AIAA 2010-4415, AIAA $5^{\text {th }}$ Flow Control Conference, 28 June-1 July, Chicago, IL, 2010.

52. Gokoglu, A.S., Kuczmarski, M.A., Culley, D.E., and Raghu, S., "Numerical Studies of an Array of Fluidic Diverter Actuators for Flow Control", AIAA 2011-3100, AIAA $41^{\text {st }}$ Fluid Dynamics Conference and Exhibit, 27-30 June, Honolulu, HI, 2011.

53. Woszidlo, R., "On the Interaction between Streamwise and Spanwise Vortices in Turbulent Shear Flow", Masters Thesis, University of Arizona, Tucson, AZ, May 2007. 


\section{REFERENCES - Continued}

54. Lucas, N., Taubert, L., Woszidlo, R., Wygnanski, I., and McVeigh, M.A, "Discrete Sweeping Jets as Tools for Separation Control", AIAA 2008-3868, AIAA $4^{\text {th }}$ Flow Control Conference, 23-26 June, Seattle, WA, 2008.

55. Cerretelli, C., Gharaibah, E., Toplack, G., Gupta, A., and Wuerz, W., "Unsteady Separation Control for Wind Turbine Applications at Full Scale Reynolds Numbers", AIAA 2009-380, 47 $7^{\text {th }}$ AIAA Aerospace Science Meeting, Orlando, FL, Jan. 2009.

56. Phillips, E., Woszidlo, R., and Wygnanski, J., "The Dynamics of Separation Control on a Rapidly Actuated Flap", AIAA 2010-4246, AIAA $5^{\text {th }}$ Flow Control Conference, 28 June-1 July, Chicago, IL, 2010.

57. Tewes, P., Taubert, L., and Wygnanski, J., "On the Use of Sweeping Jets to Augment the Lift of a $\lambda$-Wing", AIAA 2010-4689, AIAA $5^{\text {th }}$ Flow Control Conference, 28 June1 July, Chicago, IL, 2010.

58. DeSalvo, M., Whalen, E., and Glezer, A., "High-lift Enhancement using Fluidic Actuation", AIAA 2010-0863, 48 $8^{\text {th }}$ AIAA Aerospace Sciences Meeting Including the New Horizons Forum and Aerospace Exposition, Orlando, FL, Jan. 2010.

59. DeSalvo, M., Whalen, E., and Glezer, A., "Enhancement of a High-Lift Airfoil using Low-Power Fluidic Actuators", AIAA 2010-4248, AIAA $5^{\text {th }}$ Flow Control Conference, 28 June-1 July, Chicago, IL, 2010.

60. DeSalvo, M., Whalen, E., and Glezer, A., "High-Lift Enhancement Using Active Flow Control", AIAA 2011-3355, AIAA $29^{\text {th }}$ Applied Aerodynamics Conference, 2730 June, Honolulu, HI, 2011.

61. Spence, D.A., "The lift coefficient of a thin, jet-flapped wing", Proc. Roy. Soc. (London), Ser. A, Vol. 238, No. 1212, Dec. 1956, pp. 46-68.

62. Spence, D.A., "The Lift on a Thin Aerofoil with a Jet-Augmented Flap", Aeronautical Quarterly, Vol. 9, No. 3, Aug. 1956, pp. 287-299.

63. Spence, D.A., "Some Simple results for Two-Dimensional Jet-Flap Aerofoils", Aeronautical Quarterly, Vol. 9, No. 9, Nov. 1958, pp. 395-406.

64. Williams, J., Butler, S. F. J., and Wood, M. N., "The Aerodynamics of Jet Flaps", $R$. \& M. No.3304, British A.R.C, 1963.

65. Wygnanski, I. and Newman, B. G., "The Effect of Jet Entrainment on Lift and Moment for a Thin Aerofoil with Blowing", Aeronautical Quarterly, Vol. 15, 1964, pp. 122-150. 


\section{REFERENCES - Continued}

66. Woszidlo, R., Nawroth, H., Raghu, S., and Wygnanski, I., "Parametric Study of Sweeping Jet Actuators for Separation Control", AIAA 2010-4247, AIAA $5^{\text {th }}$ Flow Control Conference, 28 June-1 July, Chicago, IL, 2010.

67. Woszidlo, R. and Wygnanski, I., "Parameters Governing Separation Control with Sweeping Jet Actuators", AIAA 2011-3172, AIAA 29 $9^{\text {th }}$ Applied Aerodynamics Conference, 27-30 June, Honolulu, HI, 2011.

68. Newman, B.G., "The deflection of plane jets by adjacent boundaries - Coanda Effect", Boundary Layer and Flow Control, Lachmann, G.V. (editor), Pergamon Press, New York, 1961, pp. 232-251.

69. Personal communication with Dr. I. Wygnanski, University of Arizona, Tucson, AZ.

70. Tewes, P., Wygnanski, I., and Washburn, A., "Feedback-Controlled Forcefully Attached Flow on a Stalled Airfoil", AIAA Journal of Aircraft, doi: 10.2514/1.54616, Vol. 48, No. 3, May-June 2011, pp. 940-951.

71. Attinello, J.S., "Design and Engineering Features of Flap Blowing Installations", Boundary Layer and Flow Control, Lachmann, G.V. (editor), Pergamon Press, New York, 1961, pp. 463-515.

72. Personal communication with Roman Seele, California Institute of Technology, Pasadena, CA.

73. Drela, M., "XFOIL: An Analysis and Design System for Low Reynolds Number Airfoils", Conference on Low Reynolds Number Airfoil Aerodynamics, University of Notre Dame, 5-7 June, South Bend, IN, 1989. 